Portland State University

PDXScholar

1988

\title{
Fractional Representation Design of Dynamic Output Controllers for Multiple Time Scale Systems
}

Robert Stephen Jaffe

Portland State University

Follow this and additional works at: https://pdxscholar.library.pdx.edu/open_access_etds Let us know how access to this document benefits you.

\section{Recommended Citation}

Jaffe, Robert Stephen, "Fractional Representation Design of Dynamic Output Controllers for Multiple Time Scale Systems" (1988). Dissertations and Theses. Paper 1360.

https://doi.org/10.15760/etd.1359

This Dissertation is brought to you for free and open access. It has been accepted for inclusion in Dissertations and Theses by an authorized administrator of PDXScholar. Please contact us if we can make this document more accessible: pdxscholar@pdx.edu. 
FRACTIONAL REPRESENTATION DESIGN OF DYNAMIC OUTPUT

CONTROLLERS FOR MULTIPLE TIME SCALE SYSTEMS

by

ROBERT STEPHEN JAFFE

A dissertation submitted in partial fulfillment of the requirements for the degree of

\section{DOCTOR OF PHILOSOPHY \\ in \\ ELECTRICAL AND COMPUTER ENGINEERING}

Portland State University

C) 1988 


\section{TO THE OFFICE OF GRADUATE STUDIES:}

The members of the Committee approve the dissertation of Robert S. Jaffe presented October 23, 1987.

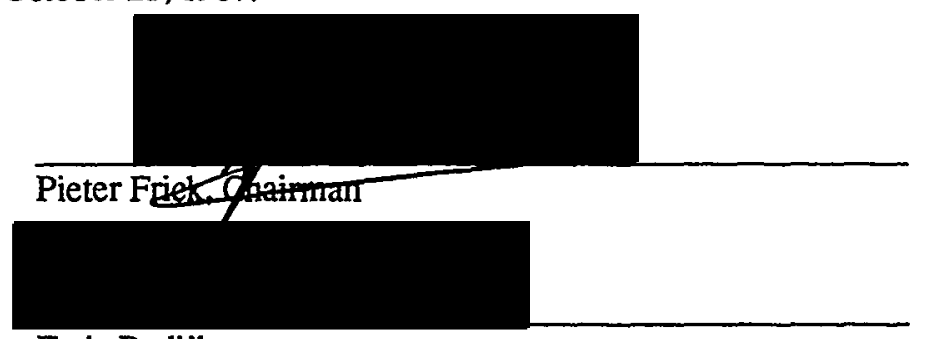

Faris Badi'i

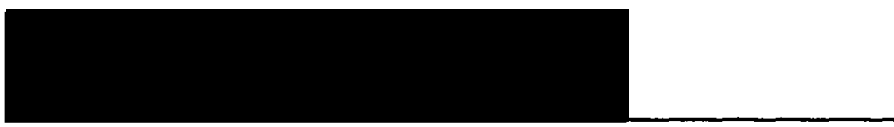

John Butler

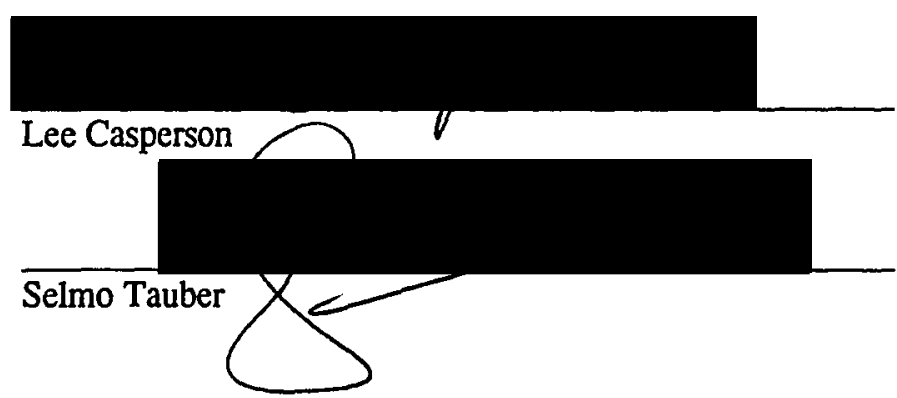

APPROVED:

Lee Casperson, Acting Chairman, Electrical and Computer Engineering

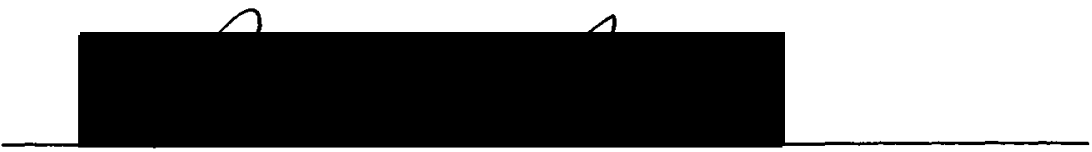

Bemard Ross, Vice Provost for Graduate Studies 
AN ABSTRACT OF THE DISSERTATION of Robert S. Jaffe for the Doctor of Philosophy in Electrical and Computer Engineering presented October 23, 1987.

Title: $\quad$ Fractional Representation Design of Dynamic Output Controllers for Multiple Time Scale Systems

\section{APPROVED BY MEMBERS OF THE DISSERTATION COMMITTEE:}

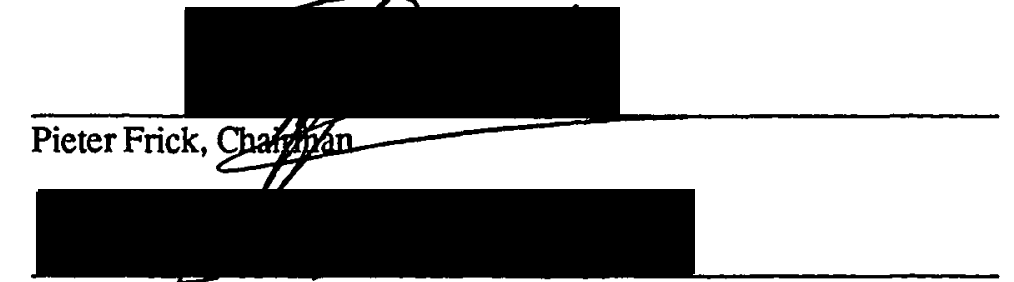

\section{Faris Badi’i}

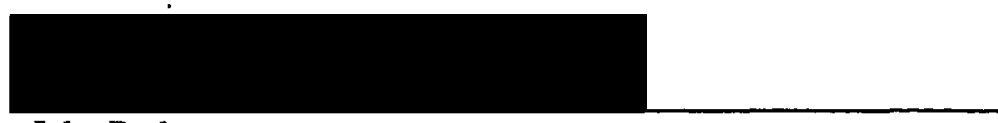

John Butler

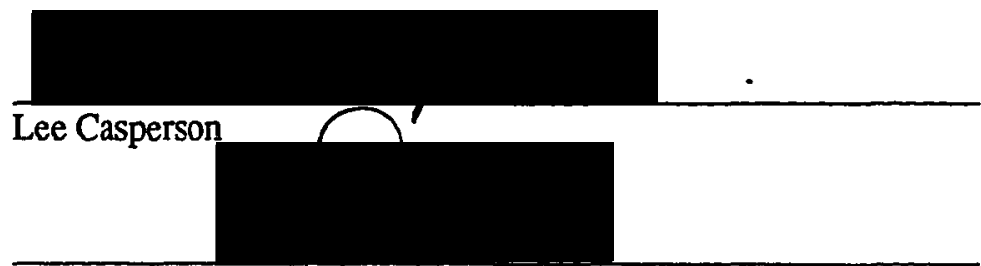

Selmo Tauber

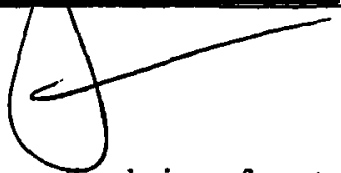

This dissertation addresses the design of controllers for multivariable finite-dimensional, linear, autonomous dynamical systems with distinct sets of slow and fast dynamics, which thus display multiple time scale behavior. It seeks, specifically, to compose overall controllers from lower-order dynamic output compensators, which are designed separately for slow and fast approximating models of the given plant. Reduction of the dynamic order of the design problem and the avoidance of numerically ill-conditioned interaction between modes of disparate orders of magnitude are among the patent advantages which pertain to such a design. 
As is well known, the explicit singularly perturbed systems, as a class, possess the multiple time scale property, while the broader class of implicit singularly perturbed systems and the multiple time scale systems are partially overlapping system classes. A composite state feedback controller scheme for the explicit singularly perturbed system has long been known. In connection with dynamic output controllers, however, only the case of the explicit singularly perturbed system with, restrictively, open-loop-stable fast dynamics has so far received attention in the literature. The dissertation, in providing a composite dynamic controller design suitable as well to the implicit singularly perturbed multiple time scale system, which furthermore is permitted to exhibit fast (or "parasitic") as well as slow (or "normal") open-loop instabilities, thus presents a more comprehensive dynamic controller strategy for this system than so far reported in the literature.

Working with multivariable transfer functions, the dissertation applies certain fractional representation techniques of modem Algebraic System Theory to the frequency domain study of the multiple time scale system. Following the work of D. W. Luse and H. K. Khalil, we replace the transfer matrix of the multiple time scale system with two or more lower-order transfer functions, each of which has validity, in its own respective frequency range, as an approximation to the first. Following the work of M. Vidyasagar, we write the rational transfer function of each of these approximating lower-order subsystems as a "fraction" over the Ring of proper and stable rational matrices. Parametrizations, in terms of "free" matrices belonging to this Ring, of the sets of stabilizing controllers for the lower-order subsystems and the corresponding achievable stable closed-loop behaviors then enable the relevant design syntheses to be achieved. In this development we exploit, specifically, a Theorem proved by Luse and Khalil concerning the relation of the closed-loop poles in a feedback configuration of multiple time scale systems to the poles in corresponding lower-order closed-loop systems. The dissertation's novel contribution thus resides in (i) interpreting the Theorem of Luse and Khalil as the outline for a possible separate- 
and-composite dynamic output controller strategy, and in (ii) adapting algebraic techniques derivative from Vidyasagar for actually realizing the putative strategy as a set of concretely implementable design procedures.

Three specific design procedures are developed and formalized in the dissertation: the first for achieving mere stabilization of the multiple time scale system, the second for the placement of slow and fast poles within specified subregions of the Complex Plane, and the third for achieving entirely arbitrary pole placement. Since these procedures derive, methodologically, from Vidyasagar's fractional representations, they are intrinsically multivariable in character. Since the procedures are validated by the Theorem of Luse and Khalil, they are applicable, in principle, to the very broadest class of linear autonomous multiple time scale systems. The dissertation presents its three design procedures in high-level-algorized form. For application to the explicit singularly perturbed system, the three procedures entail no further matrix operations than addition, multiplication, inversion and the determination of linear constant controller and observer gains, the most basic of operations in any available Control software. In connection with the implicitly singularly perturbed multiple time scale system, their concrete application requires some further computational development pertaining to the attainment of coprime factorizations of general rational matrices, a topic of independent active interest in the current literature on Control.

Elsewhere in the literature, singularly perturbed discrete time, distributed, multidimensional, time-varying and nonlinear systems have been studied. Such systems have been studied, furthermore, in several cortexts involving optimal and stochastic control, but nearly always from the time domain point of view. The dissertation tackles only the problems of robust stabilization and pole placement in the finite-dimensional, linear, autonomous case. Future work will attempt to extend its results on stabilization and pole-placement, appropriately, to some of the other general multiple time scale system classes. Further frequency domain investigations, related to the 
dissertation, may as well explore other problems pertaining to the multiple time scale systems which so far have received treatment only in the available time domain literature. 


\section{Table of Contents}

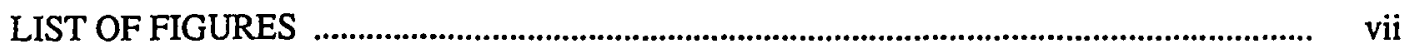

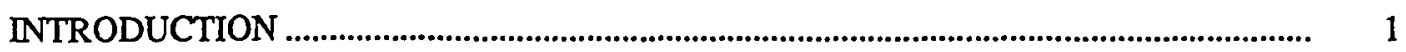

0.1 Statement of the Problem .................................................................................. 1

0.2 "Algebraic Systems Theory" and Multivariable Automatic Control ........................... 2

0.3 Outline of the Chapters of the Dissertation .................................................................... 6

\section{CHAPTER I TIME DOMAIN ANALYSIS OF THE MULTIPLE-TIME-SCALE}

SYSTEMS ........................................................ 11

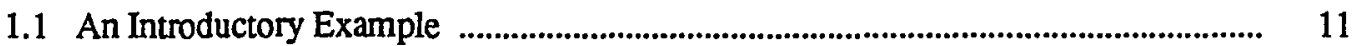

1.2 Classical Analysis of the Singularly Perturbed System ................................................ 14

1.3 Applications of the Singular-Perturbational Methods to Automatic Control ............. 21

1.3.1 Reduced-Order State Feedback Controllers ................................................. 21

1.3.2 H. K. Khalil's Reduced-Order Dynamic Controller ...................................... 25

1.4 Operator-Theoretic Analysis of the Singularly Perturbed System ........................... 30

1.4.1 Mathematical Characterization of the Implicit Singularly

Perturbed System ...................................................................... 30

1.4.2 Perturbation Theory for Linear Operators ................................................... 33

1.4.3 Algorithm for Determining the Time-Scale Hierarchies ................................. 34 
CHAPTER II FREQUENCY DOMAIN ANALYSIS OF THE MULTIPLE-TIME-

SCALE SYSTEMS

37

2.1 Review of Basic Concepts in the Algebraic Theory of Linear Dynamical

Systems

37

2.1.1 Coprime Factorization over Polynomial Matrices of the Rational

Transfer Matrix, $\mathrm{G}(\mathrm{s})$............................................................. 38

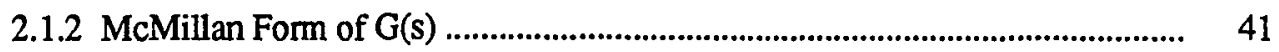

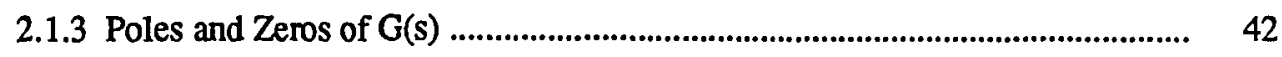

2.2 Polynomial Matrix Description of the Linear Dynamical System ............................ 44

2.2.1 Redaction of the PMD into Canonical System Matrix Form .......................... 44

2.2.2 Decoupling Zeros and Reduction of the System Matrix to Least Order .......... 46

2.2.3 Strict System Equivalence of System Matrices ............................................. 48

2.2.4 System Matrices for Closed-Loop Configurations ..................................... 50

2.3 D. W. Luse's Analysis of the Multiple-Frequency-Scale System ............................ 51

2.3.1 Algebraic Criteria for the Multiple-Frequency Scale Property ......................... 52

2.3.2 Analogy between Frequency-Domain and Time-Domain Results ................. 55

2.3.3 Representation of the Explicit Singularly Perturbed System .......................... 57

2.3.4 Representation of the General Multiple-Frequency-Scale System .................. 59

2.3.5 Derivation of Luse's Closed-Loop Theorem ............................................... 61 
CHAPTER III VIDYASAGAR'S FRACTIONAL REPRESENTATION OF

LINEAR SYSTEMS ........................................... 65

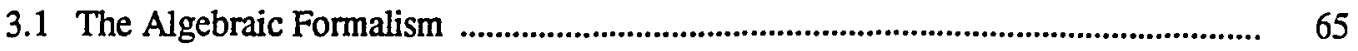

3.1.1 The Ring of Stable Transfer Functions and the Ring of Stable

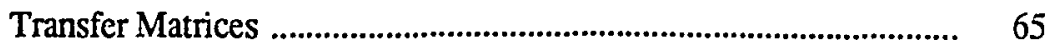

3.1.2 Parametrizations of Stabilizing Controllers and Stabilized Closed-Loop

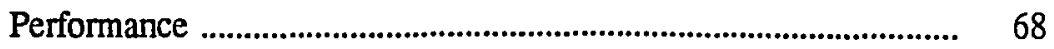

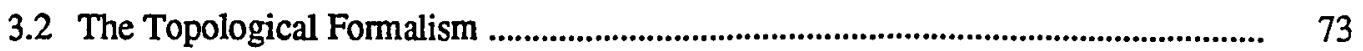

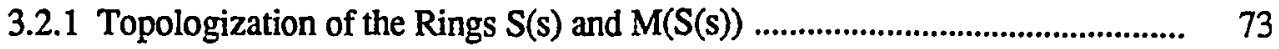

3.2.2 Graph Topology on the Ring of Arbitrary Rational Matrices, $\mathrm{M}(\mathrm{R}(\mathrm{s}))$......... 76

3.2.3 The Issue of Robust Stabilizability ............................................................ 78

3.2.4 Topology of the Explicit Singular Perturbations in the Frequency

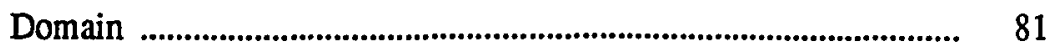

3.2.5 Strictly Proper Controllers for Singularly Perturbed Plants with

Stable Fast Dynamics ...................................................................... 83

CHAPTER IV DESIGN PROCEDURES FOR THE MULTIPLE-TIME-SCALE

SYSTEMS .......................................................... 87

4.0 Overview of the Argument of the Dissertation ........................................................... 87

4.1 Reexamination of Luse's Closed-Loop Theorem .................................................... 89

4.2 Implementation on the Explicit Singularly Perturbed System .................................. 94

4.2.1 Obtaining Doubly Coprime Factorizations from State Space

Realizations ............................................................................ 95 
4.2.2 Stabilizing Controllers and Closed-Loop Designs for the Reduced-Order

Subsystems

97

4.2.3 The Constraint of Strict Propemess and the dc-Blocking Property 101

4.2.4 Design Procedure \#1: Stabilization 107

4.2.5 Design Procedure \#2: Pole Placement within a Specified Subregion of the Complex Plane

4.2.6 Design Procedure \#3: Arbitrary Pole Placement by Algebraic

Methods 118

4.3 Applicability to the General Multiple-Time-Scale System 131

CHAPTER V CONCLUSIONS 135

5.1 Evaluation of the Dissertation's Design Procedures 135

5.2 Outline of Directions for Future Work 139

REFERENCES CITED 146 


\section{List of Figures}

1. Linear Circuit with Very Small and Very Large Parasitics ...................................... 11

2. Low, Mid and High Frequency Circuit Approximations ........................................... 12

3. Bipartite Model of the Explicit Singularly Perturbed System .................................. 17

4. Observer-Based State-Feedback Controller for the Explicit

Singularly Perturbed System ........................................................................ 24

5. H. K. Khalil's Dynamic Compensator for the Explicit

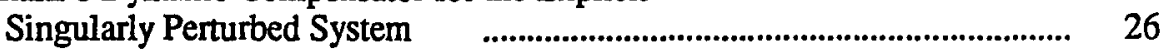

6. Basic Multivariable Feedback System Configuration ................................................ 50

7. Closed-Loop Configurations Pertaining to Two-Frequency-Scale

Systems ............................................................................................................. 61

8. Basic Multivariable Configuration Redrawn ......................................................... 68

9. Representation of the Explicit Singularly Perturbed System under Dynamic Output Compensator Control ................................................. 85

10. The Two-Frequency-Scale Feedback System and its Reduced-Order Approximating Models .................................................................................... 89

11. Representation of the Two-Frequency-Scale Controller as a Composition

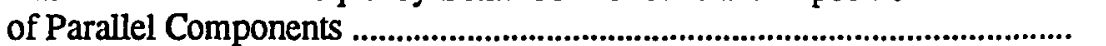

12. Subregions of Permissible Pole Placement within the Left

Half Plane

13. Paradigmatic Multivariable Closed-Loop Form

14. Individual Transfer Relationships under the Paradigmatic

Closed-Loop Form

15. Two-Stage Compensation Scheme for the General

Multivariable Plant 
16. Two-Stage Compensation Scheme for the Two-Frequency-Scale

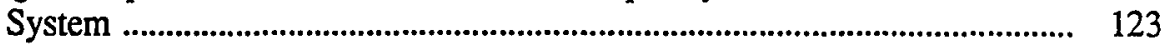

17. An Individual Transfer Relationship under the Two-Stage Compensation Scheme 


\section{INTRODUCTION}

\subsection{STATEMENT OF THE PROBLEM}

Recent developments in Mathematical Systems Theory and in the Theory of Automatic Control, which comprise "Algebraic Systems Theory", have made possible novel solutions to many problems in the analysis and design of multivariable feedback systems. This dissertation addresses the design of dynamic output controllers for a class of linear, autonomous, finitedimensional multivariable systems which have distinct sets of slow and fast dynamics and thus display multiple-time-scale behavior. The dissertation achieves a more comprehensive dynamic controller strategy for these systems than has so far been reported in the literature.

Towards identifying those dynamical systems which have the multiple-time-scale property, both a narrow class of Explicit Singularly Perturbed systems and a more inclusive class of Implicit Singularly Perturbed systems will be characterized. The Multiple-Time-Scale systems, defined dynamically, and the Singularly Perturbed systems, defined formally, will be manifested as partially overiapping system classes. The Explicit Singularly Perturbed systems will be shown as a class to possess the multiple-time scale property. The design which the dissertation achieves is applicable to the class of Explicit Singularly Perturbed systems, and as well to those Implicit Singularly Perturbed systems which display multiple-time-scale behavior. 


\section{2 "ALGEBRAIC SYSTEMS THEORY" AND MULTIVARIABLE AUTOMATIC CONTROL}

The mathematical framework presupposed by Classical Control Theory was the Laplace Transform and the Theory of Functions of a Complex Variable. The open-loop gain, or "transfer function", of a linear autonomous finite-dimensional system was expressed within this framework as a rational function of the complex frequency variable. Abstract blocks displaying the transfer functions were used to represent the effect of these systems on signals passing through them. Diagrams which featured closed loops around these blocks then built the transfer functions specifically into the description of feedback control systems. Thereupon, many of the powerful results already achieved in the mathematical context of the Theory of Functions of a Complex Variable could be adapted to Control System analysis and design. The notions of Gain Margin and Phase Margin, Root Locus and Nyquist Criterion, the comerstone concepts of the classical frequency-domain theory, represent such adaptations from Complex Variable Theory.

These classical concepts, having been defined most basically as single-loop quantities, were not evidently applicable to multivariable systems, in which simultaneous variations in several loops might occur. Moreover, the transfer-function algebra of the classical approach, straightforward though it appeared to be, worked only under the provision that all systems be initially relaxed. Additionally, the pole-zero cancellation problem, i.e. the problem of hidden modes, emerged as a circumstance which the classical transfer-function theory could not handle in any unified and theoretically satisfying way. Heuristic rules were devised for working around the cancellations in the single-input-single-output (SISO) or scalar case, but the multiple-inputmultiple-output (MIMO) or multivariable version of the cancellation problem remained intractable to anything in the array of classical methods. In the upshot, Classical Control Theory succeeded in providing a coherent though not entirely unified frequency-domain approach -- for scalar systems only. 
The celebrated State-Space Approach of the 1960's based itself on time-domain rather than frequency-domain analysis. It laid specific emphasis upon the time-response of a canonical system of first-order ordinary differential equations which had already been extensively studied in the mathematical literature. This canonical form provided a more rigorous and more precise framework than the classical transfer-function-algebra for the study of dynamical systems. It enabled the "State Variable" concept to be introduced into Control Theory. Interpretively speaking, the State variables enable what is "inside" the system to be displayed, and they build into the analysis of these systems a factor relating to their histories. The concept of State thus made possible a deeper penetration of the structure of control systems than the classical theory could achieve. The State-Space Approach could handle both the situation of initially non-relaxed systems and the situation of hidden modes. It permitted the formulation and solution of a number of problems of Automatic Control that were not open to the classical methods. State Observer, Linear State Feedback Controller, Optimal Linear Quadratic Regulator and Kalman Filter are among the well known achievements of the State Variable Approach.

Multivariable systems are treated, in principle, in exactly the same way as single-loop systems under the State Variable Approach. That is to say, MIMO problems and SISO problems receive exactly the same formulation -- a feature in itself possessive of great elegance. Nonetheless, tools for the actual execution of these problems in the MIMO case were not entirely forthcoming from among the State-Space methods of the 1960's and early 1970's. For the most part, the mathematical machinery that well enough served the State Variable Theory in the SISO case became cumbrous and faltered in the attempt at mulivariable generalization. To be sure, solutions for the multivariable state observer and for multivariable linear state feedback have been achieved. But the State Variable methods by themselves remain ill suited to the design of dynamic controllers for multivariable plants. 
Currently, a theory for multivariable feedback systems is being developed which enables many of the results of the State Variable Approach to be brought back within the purview of an improved and generalized transfer-function analysis. The new and unifying framework uses for its primary mathematical machinery an algebraic formalism more precise and more detailed than was hitherto available. Clearly, the multivariable systems possess more subtle algebraic properties than their scalar counterparts. An algebraic machinery which can properly generalize to the MIMO case the classical concepts of pole, zero and hidden mode, and can as well identify new frequency-domain concepts which emerge first only in the multivariable context, is currently being developed.

The truly pioneering breakthrough towards a modem algebraic theory for multivariable linear dynamical systems was H.H. Rosenbrock's work in State-Space and Multivariable Theory [27] in the early 1970's. Rosenbrock generalized the SISO into the MIMO case by expressing the rational transfer matrix of a multivariable system as a particular type of fraction over polynomial matrices. This innovation, the Matrix-Fraction Description (MFD) of a MIMO transfer function, has spawned a set of developments which comprise the "Fractional-Representation Approach" to multivariable control system analysis and design. Rosenbrock made heavy use in his work of the Smith and McMillan canonical forms to reveal the deep structures of polynomial and rational matrices, respectively. Thanks to his in-depth study, the system-theoretic significance of various manipulations of these matrices could be identified and understood. This knowledge could then be exploited for purposes of analysis and design of multivariable feedback control systems.

Rosenbrock studied a class of systems with dynamical behavior described by a set of differential equations less restrictive in form than the canonical equations required for State Variable analysis. Each of these more general differential systems receives a canonical Polynomial Matrix Description (PMD) -- i.e. a description in terms of matrices over the Ring of polynomials in the differential operator. Rosenbrock aiso uses a peculiarly constructed individual matrix, the 
"System Matrix", to display the Polynomial-Matrix-described system. Within the unifying framework of the System Matrix, State-Variable and Matrix-Fraction representations are treated as special cases of the Polynomial Matrix description. The System Matrix thus serves as an algebraic bridge between time-domain dynamical-equation and frequency-domain input-output methodologies, the distinction between the two no longer being hard and fast. The comprehensive theory which derives from Rosenbrock's approach thus highlights similarities rather than differences between Classical and State Variable approaches. Rosenbrock's approach lends itself, resultantly, to a more effective choice of methods for analysis and techniques for design than where the study is confined to a single domain alone.

In its most abstract definition, "Algebraic Systems Theory" is a branch of mathematics which views systems as functional morphisms between sets endowed with particular algebraic structures. For example, linear systems whose input, output and state sets each admit a Group structure can be viewed as Group homomorphisms. When different classes of control system are examined under different analytical frameworks, different algebraic structures arise. The familiar class of finite-dimensional, linear, time-invariant systems has been viewed under the guise of a Field structure (to guarantee the "nice" properties of its transfer-function algebra) in the Classical approach and under the guise of an Euclidean Vector-Space structure (to exploit the "nice" geometry of its State space) in the State Variable Approach. More modem approaches [1] tend to view these same systems as a class of Module morphisms over a Ring of polynomials in the differential operator. In many cases, the appropriate choice of an algebraic structure enables classes of discrete as well as continuous time, distributed as well as lumped parameter, time-varying as well as autonomous, and nonlinear as well as linear systems to be studied under corresponding algebraic frameworks.

Needless to say, any abstract way of doing Mathematical Systems Theory invites the 
tradeoff between generality and explicitness. Many system-theoretic concepts can indeed be defined using only a minimal amount of algebraic structure. But when the frameworks are too highly abstract, only very inexplicit results are derivable under them. The chapters which follow draw upon Rosentbrock's work and upon a more recent version of the Fractional-Representation Approach which stems from the work of M. Vidyasagar and his collaborators [29-34]. Both Rosenbrock and Vidyasagar do Algebraic Systems Theory at a level which is specific enough to yield explicit solutions to definite problems. Since the proofs in Algebra nearly always take constructive form, there is already implied in theoretical work done in the style of Rosenbrock and Vidyasagar a framework in which definite computations can be performed. Software development and hardware implementation can therefore follow with relative swiftness after theoretical results are derived under the umbrella of the modern Algebraic approaches to Automatic Control.

\subsection{OUTLINE OF THE CHAPTERS OF THE DISSERTATION}

Chapter I is devoted to the time-domain analysis of the explicit singularly perturbed system and to the time-domain analysis of those implicit singularly perturbed systems which display multiple-time-scale behavior. Through methods which are familiar from the classical study of Differential Equations, a higher order system of the explicit singularly perturbed type is shown to be replaceable by two or more lower-order subsystems, each of which has validity, in its own respective time-scale, as an approximation to the given one. The chapter briefly reviews some of the applications of this singular-perturbational technique which have been made in the context of Automatic Control. Specifically, a number of strategies for compensating singularly perturbed plants with reduced-order controllers of state-feedback, observer-based-state-feedback, staticoutput-feedback and dynamic-output-feedback types are investigated. The dynamic-outputfeedback strategy here reviewed is limited in use to one particularly restrictive standard 
application. In subsequent chapters, the modem Algebraic approach to multivariable control system analysis and design will permit a more comprehensive dynamic controller strategy for the singularly perturbed plant to be devised .

The focus of Chapter I being time-domain, all the methods used in it derive from State Variable Analysis. Before the modern Algebraic approach can be applied to the multiple time scale systems, a frequency-domain characterization needs to be given. Chapter II, accordingly, regards these systems now as multiple-frequency-scale systems, with transfer matrices which are replaceable by two or more reduced-order transfer matrices, each of them having validity, in its own respectively appropriate frequency-range, as an approximation to the first. Chapter II thus performs a kind of singular-perturbational analysis in the frequency domain -- the system's approximate behavior in different frequency-ranges now substitutes for approximate behavior on different time-scales.

The work of D.W. Luse [18-21] provides a framework in which the multiple-frequencyscale behavior of finite-dimensional, linear, autonomous multivariable systems can be studied. Luse establishes a set of algebraic conditions on the transfer matrices of these systems which guarantee multiple-frequency-scale behavior, and thus effectively identify, from the frequency domain point of view, the broad class of implicit singularly perturbed systems which possess the multiple-time-scale property. Not surprisingly, the narrower class of explicit singularly perturbed systems displays a still more specific characteristic structure in the frequency domain.

Chapter II includes a review of those basic concepts and techniques of modem multivariable Algebraic Systems Theory which are needed for the development of Luse's work. Rosenbrock's Polynomial-Matrix description of the linear dynamical system and his redaction of this description into canonical System-Matrix form are covered. Rosenbrock worked with matrices of polynomials or rational functions in the differential operator with coefficients taken from Real or Complex Fields. The description of the multiple-frequency-scale system involves 
matrices of polynomials or of rational functions in the complex frequency domain whose coefficients belong to a certain Field of functions of a perturbation-parameter. Luse shows how systems of the latter type of description can still be treated within Rosenbrock's basic framework.

The culminating point in the exposition of Luse's work is a Theorem concerning the location of closed-loop poles in a feedback configuration of two-frequency-scale systems. For the problem which is here at hand, Luse's Theorem has great importance, since under one interpretation this Theorem suggests a dynamic output controller design strategy for the multiplefrequency-scale systems. The role of the next chapter is to provide through the work of $M$. Vidyasagar [29-34] a set of algebraic tools which are capable of realizing Luse's suggested strategy as a concretely implementable design procedure for the given class of systems.

Chapter III studies the Fractional-Representation approach to control system analysis and design which is presented in Vidyasagar's Control System Synthesis [32]. The Ring of transfer matrices of proper, stable, multivariable, finite-dimensional, linear, time-invariant systems provides the basic algebraic structure in Vidyasagar's work. Vidyasagar produces a parametrization, in terms of a "free" matrix over this Ring, of the set of all dynamic controllers which have the capability to stabilize a given multivariable plant. He provides a similar parametrization of the set of all closed-ioop behaviors which the compensated system has the capability to perform. An algebraic design procedure for the general class of multivariable linear time-invariant plants is then derived from these convenient parametrizations.

The problem of stabilizing a singularly perturbed system bears similarity to the problem of robustly stabilizing an imperfectly-known or variable plant. Vidyasagar in fact calls attention to the singularly perturbed system in the context of his general discussion of robust stabilization. Into any rigorous discussion of robustness, topological categories must be introduced. Vidyasagar has topologized, accordingly, both the specific Ring of proper stable MIMO transfer matrices and the larger Ring of matrices over the Field of arbitrary rational functions. Through the prism of 
these topologies, additional insights into the structure of singular perturbations in the frequency domain are then reflected.

Chapter IV goes for the first time beyond the current state-of-the-art in the design of dynamic output controllers for the multiple-time-scale systems; in this chapter resides the dissertation's unique and novel achievement. Its innovation consists in interpreting Luse's Theorem conceming the location of closed-loop poles in a feedback connection of twofrequency-scale systems as the outline for a dynamic controller design strategy, and then applying algebraic techniques which derive from Vidyasagar's parametrizations of the stabilizing compensators to achieve a set of concretely implementable design procedures. In the implementation of these procedures, a singularly perturbed controller is composed from two lower-order dynamic compensators, which are designed separately for the "slow" and "fast" approximating models of the given plant, yet are not entirely independent and uncoupled. Through the strategem of requiring one of these controllers to have the low-pass property of strict properness and the other to have the dc-blocking high-pass property, the satisfaction of the requisite coupling condition is ensured. This strategem is shown to be incorparable into Vidyasagar's algebraic framework in a very natural way; consequently, the design procedures are not burdened with any set of complicating ad hoc constraints on behalf of the coupling condition.

The singularly perturbed system is presented canonically in State Variable form, yet the algebraic machinery applies instead to systems which present themselves in the form of MatrixFraction description. For this reason, in Chapter IV time-domain and frequency-domain methods both come into play. Translation between the two domains is enabled by a Lemma which obtains the requisite Matrix-Fraction Description from the State Variable representation of a given system. Matrix-Fraction descriptions of the approximating lower-order subsystems of the given plant then play the primary roles in Chapter IV's design procedures. Once these descriptions have been attained, the stabilization of the plant can be immediately achieved, with an expression 
for the full range of design freedom consistent with the inaintenance of stability following closely by Vidyasagar's methods. When criteria more specific than mere stabilization are specified, this range of freedom must be appropriately narrowed. Towards the placement of poles within specified subregions of the Complex Plane, and towards the achievement of arbitrary poleplacement for the closed-loop singularly perturbed system, two further design procedures are developed and formalized.

Chapter IV includes an extended example which illustrates the basic features of the three design procedures. The procedures' most serious drawbacks include their failure to place the zeros of the closed-loop system, their failure to minimize the dynamic order of the stabilizing controllers and their failure to guarantee the stability of the stabilizing controllers themselves. The procedures' greatest merits are their genuinely multivariable character and their adaptability to the broadest class of linear autonomous multiple-time-scale systems. The concluding chapter, Chapter V, examines the grounds for improvement of Chapter IV's design procedures and sketches a number of more general directions in which the findings of the dissertation might be extended in future work. 


\section{CHAPTER I}

TIME-DOMAIN ANALYSIS OF THE MULTIPLE-TIME-SCALE SYSTEMS

\subsection{AN INTRODUCTORY EXAMPLE}

The following circuit appears in an early paper by Desoer and Shensa on the subject of "Networks with Very Small and Very Large Parasitics" [4]:

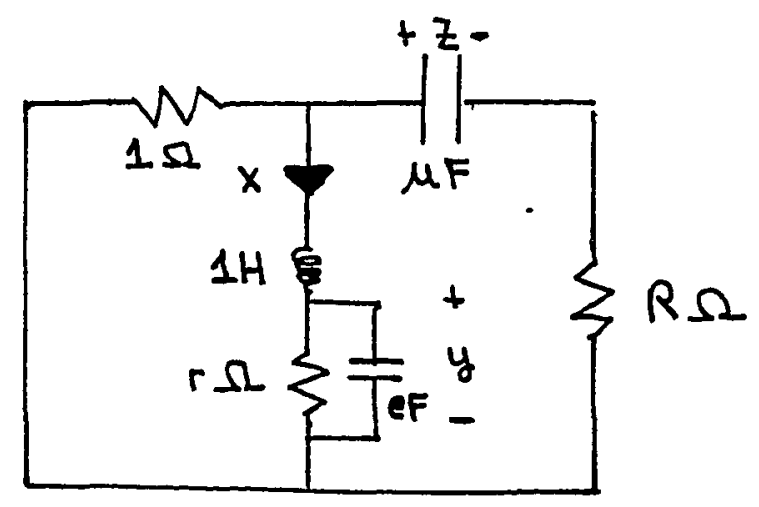

Figure 1

The circuit has three energy-storing elements, a large coupling capacitance of $u$ Farads, a small stray capacitance of e Farads, and an inductance of 1 Henry. Coupling capacitor voltage, stray capacitor voltage and inductor current are most naturally chosen for State Variables in the State Space description of the circuit. The State equations of the third-order system are then given by: 


$$
\left[\begin{array}{c}
\dot{x} \\
e \dot{y} \\
u \dot{u}
\end{array}\right]=\left[\begin{array}{ccc}
-R /(R+1) & -1 & 1 /(R+1) \\
1 & -1 / r & 0 \\
-1 /(R+1) & 0 & -1 /(R+1)
\end{array}\right]\left[\begin{array}{l}
x \\
y \\
z
\end{array}\right]
$$

It is conventional in Circuit Analysis to make the following approximations. $u$ is extremely large, thus the impedance of the coupling capacitor is virtually zero at middle and high frequencies and is $0(1)$ at low frequencies. e is extremely small, thus the impedance of the stray capacitor is virtually infinite at low and middle frequencies and is $0(1)$ at high frequencies. The impedance of the inductor is virtually zero at low frequencies and is virtually infinite at high frequencies, while it is $0(1)$ at middle frequencies. The given circuit is thus conventionally represented at low, mid- and high frequencies by the following reduced-order approximating models:

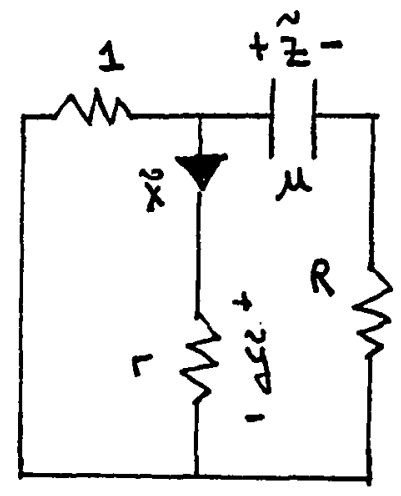

Figure 2a

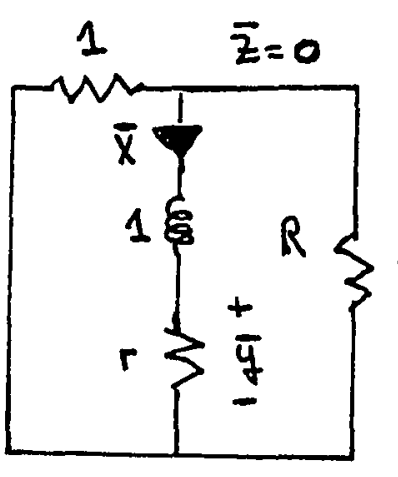

Figure $2 b$

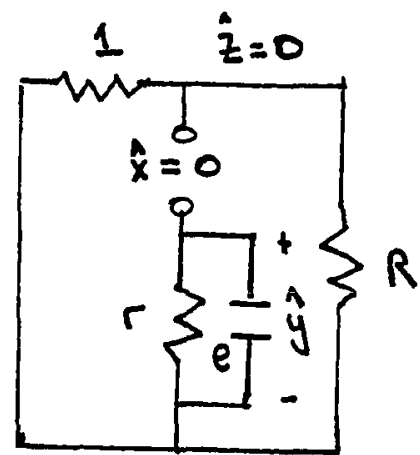

Figure 2c

The sets of equations corresponding to the reduced-order approximating models are given by:

$$
\begin{array}{ccc}
u \dot{\bar{z}}=-(1 /(R+1)) \bar{x}-(1 / R+1)) \bar{z} & \dot{\bar{x}}=-(R /(R+1)) \bar{x}-\bar{y} & e \dot{y}=-(1 / r) \hat{y} \\
\tilde{x}=(1 / r) \tilde{y} & \bar{x}=-(1 / r) \bar{y} & \hat{x}=0 \\
(R /(R-1)) \bar{x}+\tilde{y}=(1 /(R+1)) \tilde{z} & \bar{z}=0 & \hat{z}=0
\end{array}
$$

The "fast" variable, $y$, corresponding to the stray capacitor, has associated with it slow and nor- 
mal and fast components, denoted by $\tilde{y}, \bar{y}$ and $\hat{y}$, respectively. The "normal" variable, $x$, corresponding to the inductor, has both slow and normal components, $\bar{x}$ and $\bar{x}$ associated with it. The "slow" variable, $\mathrm{z}$, corresponding to the coupling capacitor, has only the associated slow component $\bar{z}$. Approximately: $z=\tilde{z}, x=\tilde{x}+\bar{x}$, and $y=\tilde{y}+\bar{y}+\hat{y}$. The low-frequency equations dynamically determine the slow variable $\tilde{z}$, to which the slow components $\tilde{x}$ and $\tilde{y}$ of the normal and fast variable are algebraically coupled. The mid-frequency equations dynamically determine the normal component $\bar{x}$ of the normal variable, to which a normal component $\bar{y}$ of the fast variable is algebraically coupled. The high-frequency equation dynamically determines the fast component of the fast variable.

The low-frequency equations are as if written in a slow time-scale, in which the slow components' dynamics normally evolve; but the normal and fast components are so fast in this time scale that their evolutions complete themselves instantaneously. The mid-frequency equations are as if written in a normal time-scale, in which the normal components evolve dynamically; but the fast component is so fast in this time scale that its evolution is instantaneously completed, while the slow components are so slow that their evolutions do not even effectively commence. The high-frequency equation is as if written in a fast time-scale, in which the fast component of the fast variable dynamically evolves; but all other components are so slow in this time scale that they effectively remain constant. Effectively, through the familiar circuit-theoretic approximations, the complete dynamics of the given third-order circuit are replaced by the dynamics of three separate first-order systems, which evolve in different time-scales.

In their paper, Desoer and Shensa provide the rigorous mathematical analysis which validates the above conventional use of the reduced-order models to approximate the given circuit. These authors establish formally that the natural frequencies associated with the given circuit are asymptotically -- i.e. as the parameter $u$ approaches infinity and the parameter e approaches zero - the natural frequencies of the three reduced-order networks. For this reason, if low-frequency, 
mid-frequency and high-frequency networks all are stable, the given circuit is stable for all $\mathrm{e}$ in $\left(0, e^{*}\right)$ and for all $u$ in $\left(u^{*}, \infty\right)$, for some upper bound $e^{*}$ and some lower bound $u^{*}$. Conversely, instability of any one of the approximating networks determines the instability of the given circuit for all values of $e$ less than some upper bound $e^{*}$ and all values of $u$ greater than some lower bound $u^{*}$.

It is well-known that even when a circuit like the given one is stable at middle frequencies, low-frequency instabilities can still cause "motorboating" and high-frequency instabilities can still cause "singing". The above results of Desoer and Shensa are useful, because they provide a way to identify these unstable conditions, short of the exact, full-order dynamical analysis of the given circuit itself. Chapter I, devoted to the time-domain analysis of a very broad class of systems which shall be identified as "multiple-time-scale", both deepens and broadens these basic early results which were attained by Desoer and Shensa.

\subsection{CLASSICAL ANALYSIS OF THE SINGULARLY PERTURBED DIFFERENTIAL SYSTEM}

The Analytical Theory of Differential Equations, within classical mathematics, contains a standard literature on Singularly Perturbed Differential Systems. Though time-varying and nonlinear singularly perturbed systems have been studied, the present discussion will be limited to the linear autonomous case. A linear autonomous system is, by definition, explicitly singularly perturbed if it has the form:

$$
\begin{gathered}
\dot{x}=A_{11} x+A_{12} z+B_{1} u \\
\dot{z} z=A_{21} x+A_{22} z+B_{2} u \\
y=C_{1} x+C_{2} z \\
x\left(t_{0}\right)=x_{0} \quad z\left(t_{0}\right)=z_{0} \\
x \text { in } R^{n}, z \text { in } R^{m}, u \text { in } R^{p}, y \text { in } R^{q}
\end{gathered}
$$


In system (3), e is understood to be a small positive scalar parameter. Since it multiplies the derivatives of some of the states, this positive scalar roughly represents the ratio of speeds of slow and fast dynamics within the system.

A goal of singular-perturbational analysis is to draw conclusions about the behavior of system (3) for small values of the parameter, on the basis of a simplified model of that system which is afforded the mathematical convenience of having the parameter set to zero. This simplified model will consist of two parts. In one part of the model, the slow dynamics of system (3) are approximated, by setting the parameter to zero in a way that neglects the fast dynamics. And in the other part of the model, the fast dynamics are approximated, by setting the parameter to zero in a way that neglects the slow dynamics. The simplified model will thus have effectively separated the slow and fast dynamics of the given system, and e will have played the role of "perturbation parameter" within the framework of the analysis.

For very small values of the parameter, the fast modes are so fast as to be virtually instantaneous. When, for mathematical convenience, the parameter is set to zero in system (3), the second, or "fast", dynamical equation degenerates into an algebraic equation, which, assuming $A_{22}$ nonsingular, can be solved for $z$, as follows:

$$
z=-A_{22}^{-1} A_{21} x-A_{22}^{-1} B_{2} u
$$

With $\mathrm{z}$ eliminated, (3) reduces to the system:

$$
\begin{gathered}
\dot{\bar{x}} \equiv\left(A_{11}-A_{12} A_{22}^{-1} A_{21}\right) \bar{x}+\left(B_{1}-A_{12} A_{22}^{-1} B_{2}\right) \bar{u} \equiv A_{0} \bar{x}+B_{0} \bar{u} \\
\bar{z}=-A_{22}^{-1} A_{21} \bar{x}-A_{22}^{-1} B_{2} \bar{u} \\
\bar{y}=\left(C_{1}-C_{2} A_{22}^{-1} A_{21}\right) \bar{x}-C_{2} A_{22}^{-1} B_{2} \bar{u} \equiv C_{o} \bar{x}+D_{0} \bar{u} .
\end{gathered}
$$

System (5) is required to take the initial condition: $\bar{x}\left(t_{0}\right)=x_{0}$. Though the system is solvable for both $\bar{x}$ and $\bar{z}$, as a dynamical system it has only order $n$. System (5) will heretofore be regarded 
as an approximate "reduced-order model" for the slow dynamics of system (3). In (5), the fast dynamics of (3) are effectively disregarded.

It is next sought to approximate the fast dynamics of (3) by neglecting its slow dynamics. With this end in view, a fast time variable is introduced:

$$
\tau=\left(t-t_{0}\right) / e .
$$

$\tau$ is "fast" because when $\tau$ is $O(1),\left(t-t_{0}\right)$ is still only $O(e)$; and when $\left(t-t_{0}\right)$ reaches $O(1), \tau$ is already $0(1 / e)$. In the fast time scale, system (3) is given by:

$$
\begin{gathered}
d x / d \tau=e A_{11} x+e A_{12} z+e B_{1} u \\
d z / d \tau=A_{21} x+A_{22} z+B_{2} u .
\end{gathered}
$$

In this time scale, for very small values of the parameter, the slow dynamics are so slow as to be virtually constant. Setting the parameter to zero in this system, for mathematical convenience, regards them specifically as constant, and thereby effectively disregards them from the dynamical point of view.

Next, the following m-dimensional system in the fast time scale is introduced:

$$
\begin{gathered}
\mathrm{d} \hat{\mathrm{z}} / \mathrm{d} \tau=\mathrm{A}_{22} \hat{\mathrm{z}}(\tau)+\mathrm{B}_{2} \hat{\mathrm{u}}(\tau) \\
\hat{\mathrm{Y}}(\tau)=\mathrm{C}_{2} \hat{\mathrm{z}}(\tau) \\
\hat{\mathbf{z}}_{\mathrm{o}}=\mathrm{z}_{\mathrm{o}}-\overline{\mathrm{z}}\left(\mathrm{t}_{\mathrm{o}}\right) .
\end{gathered}
$$

The initial condition for $\hat{z}$ matches the initial disparity between the "true" state $z$ of system (3) and the state $\bar{z}$ of system (5). Assuming the stability of the $A_{22}$ matrix, the "true" $z$ (t) has both: (i) a slow component (already approximated by $\bar{z}$ ) which contributes to the quasi-steady-state of the system's reduced-order model, and (ii) a fast component which decays after an initial transient period. System (8) will henceforth be taken as an approximate equation for the fast component of 
2, which is regarded as a function of the fast time variable. Due to its matching of conditions at the boundary time-value of $t=t_{0}$, system (8) is spoken of as providing a "boundary-layer correction" to system (5)'s "reduced-order model" of system (3). Taken together, (5) and (8) comprise that simplified bipartite model of system (3) which has been sought. In block-diagram form, this simplified model may be represented by Figure 3:

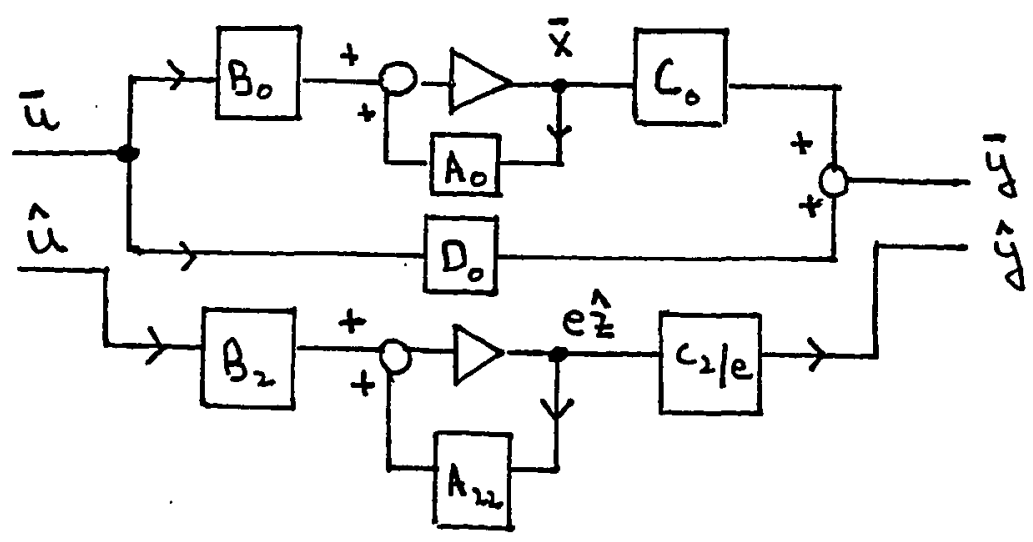

Figure 3

The input $u$ to system (3) has been separated into an input $\bar{u}$ which drives subsystem (5) and an input $\hat{u}$ which drives subsystem (8). It is assumed that $u(t)$ contains two classes of components, with respectively slow and fast dynamics, which are transmitted through system (3) accordingly.

The reasoning which has led to this point in the exposition has been basically heuristic in nature. In the classical mathematical treatment of the singularly perturbed system, such reasoning receives its rigorous validation through the construction of power series expansions about $e=0$, in terms dependent on $t$ and in terms dependent on $\tau$, for each of the quantities $x, \bar{x}, z, \bar{z}$, and $\hat{z}$. The limiting values of these series as the parameter asymptotically approaches zero are then compared. Through this procedure, the following approximations are determined to be valid, for all $e$ in some sufficiently small positive range and for all $t$ in $\left[t_{0}, \infty\right)$ : 


$$
\begin{gathered}
x(t)=\bar{x}(t)+O(e) \\
z(t)=\bar{z}(t)+\hat{z}(\tau)+O(e) \\
y(t)=\bar{y}(t)+\hat{y}(\tau)+O(e)
\end{gathered}
$$

This method of matched asymptotic expansions in separate time scales is capable of yielding still better approximations, in principle up to the order $O\left(\mathrm{e}^{\mathrm{k}}\right)$ for any positive integer $\mathrm{k}$ that is specified.

For the linear autonomous explicitly singularly perturbed system that has been under consideration, there is a purely algebraic way to show the dependence of the system's dynamic behavior on two separate time scales. Purely by a change of basis for the State Space, i.e. by a linear transformation of variables, slow and fast subsystems of (3) can be completely decoupled -in which connection, no explicit reference to the "reduced-order model" and "boundary-layer correction" of the previous discussion needs to be made.

Suppose that the given state vector $\left(x^{2} z^{2}\right)^{t}$ is to be transformed into the new state vector $\left(v^{t} w^{t}\right)^{t}$. First, $z$ will be eliminated, through introduction of the new variable $w=z+L x$. In terms of $(x, w)$ the matrix of State is given by:

$$
\left[\begin{array}{c}
\dot{x} \\
\dot{w}
\end{array}\right]=\left[\begin{array}{ll}
A_{11}-A_{12} L & A_{12} \\
L A_{11}-A_{12} L+A_{21} / e-\left(A_{22} / e\right) L & L A_{12}+A_{22} / e
\end{array}\right]\left[\begin{array}{l}
x \\
w
\end{array}\right]
$$

We shall suppose that $L(e)$ has been chosen as a solution to the algebraic matrix equation:

$$
\mathrm{LA}_{11}-\mathrm{A}_{12} \mathrm{~L}+\mathrm{A}_{21} / \mathrm{e}-\left(\mathrm{A}_{22} / \mathrm{e}\right) \mathrm{L}=0
$$

By (11), the lower left submatrix in (10) becomes null, and the system is partially decoupled.

In the second stage of the transformation, $\mathrm{x}$ will be eliminated, by introduction of the new 
variable $v=x-M w$. In the $(v, w)$ coordinate system, the matrix of State is given by:

$$
\left[\begin{array}{c}
\dot{v} \\
\dot{w}
\end{array}\right]=\left[\begin{array}{ll}
A_{11}-A_{12} L & A_{11} M-A_{12} L M+A_{12}-M L A_{12}-M A_{22} / e \\
0 & A_{22} / e+L A_{12}
\end{array}\right]\left[\begin{array}{l}
v \\
w
\end{array}\right]
$$

When, finally, we suppose $M(e)$ to have been chosen to satisfy the matrix equation:

$$
\left(A_{11}-A_{12} L\right) M+A_{12}-M\left(A_{22} / e+L A_{12}\right)=0
$$

the upper right submatrix in (12) becomes null, and the system is completely decoupled.

We are interested, as before, in being able to approximate the behavior of system (3) for small positive values of the parameter. From (11), L can be approximated for small e by:

$$
L=A_{22}^{-1} A_{21}+O(e)
$$

An approximate system description for (3) in the decoupled coordinate system is thus given by:

$$
\left[\begin{array}{c}
\dot{v} \\
e \dot{w}
\end{array}\right]=\left[\begin{array}{ll}
A_{11}-A_{12} A_{22}^{-1} A_{21}+0(e) & 0 \\
0 & A_{22}+0(e)
\end{array}\right]\left[\begin{array}{l}
v \\
w
\end{array}\right] \equiv\left[\begin{array}{ll}
A_{0}+0(e) & 0 \\
0 & A_{22}+0(e)
\end{array}\right]
$$

The net transformation is representable by:

$$
\left[\begin{array}{l}
v \\
w
\end{array}\right]=\left[\begin{array}{ll}
I-M L & -M \\
L & I
\end{array}\right]\left[\begin{array}{l}
x \\
z
\end{array}\right] \quad\left[\begin{array}{l}
x \\
z
\end{array}\right]=\left[\begin{array}{ll}
I & M \\
-L & I-L M
\end{array}\right]\left[\begin{array}{l}
w \\
y
\end{array}\right]
$$

(16) is a nonsingular linear transformation, a similarity transformation. Under such a transformation, the eigenvalues are preserved. Since, furthermore, the eigenvalues permute continuously with continuous permutations of the elements of the matrix, it follows that system (3) has, for sufficiently small e: (i) $n$ small eigenvalues which are close to the eigenvalues of $A_{0}$, and (ii) $\mathrm{m}$ large eigenvalues which are close to $1 / \mathrm{e}$ times the eigenvalues of $A_{22}$. This important 
result merits being stated formally as a theorem:

Theorem 1: Assuming that $A_{22}$ is nonsingular, the set of eigenvalues of system (3), for sufficiently small $e$, is approximated by the union of:

(i) $(1+0(e))$ times the set of eigenvalues of $\left(A_{11}-A_{12} A_{22}^{-1} A_{21}\right)$ and

(ii) $(1+0(\mathrm{e}))$ times the set of eigenvalues of $\left(\mathrm{A}_{22} / \mathrm{e}\right)$.

Clearly, two distinct sets of dynamics are here involved. The small eigenvalues are associated with slow modes and large time constants, and the large eigenvalues with fast modes and small time constants. The dependence of system (3)'s behavior on two separate time-scales has been reestablished, this time without any reference to its "reduced order" and "boundary layer" approximating subsystems, through a study of the spectrum of its Characteristic Matrix.

Several significant corollaries pertaining to the stability of singularly perturbed systems follow immediately from Theorem 1:

Corollary 1: If all the eigenvalues of $A_{0}$ and all the eigenvalues of $A_{22}$ have negative real parts, then there exists an $\mathrm{e}^{*}$ such that all the eigenvalues of system (3) have real parts for all $e$ in $\left(0, e^{*}\right)$.

Corollary 2: If at least one of the eigenvalues of $\mathrm{A}_{0}$ or at least one of the eigenvalues of $A_{22}$ has positive real part, then there exists an $e^{*}$ such that at least one eigenvalue of system (3) has positive real part for all $\mathrm{e}$ in $\left(0, \mathrm{e}^{*}\right)$.

Corollary 3: System (3) is asymptotically stable for sufficiently small values of $e$ if and only if its reduced-order system (system (5)) and its boundary-layer system (system (8)) are asymptotically stable. 


\subsection{APPLICATIONS OF 'THE SINGULAR-PERTURTBATIONAL METHODS TO AUTOMATIC CONTROL}

We now suppose that system (3) represents a plant which is to be regulated. The discussion moves from the general mathematical theory of the singularly perturbed system into the application of time-domain singular-perturbational methods to the problems of Automatic Control.

In the Control context, the separation of the plant's dynamics into slow "reduced-order" and fast "boundary-layer" subsystems invites the possibility that separate control laws in each of two distinct time scales might be devised for the lower order subsystems, and then combined appropriately to form a composite controller for the given plant. When there is interaction between the very large and very small eigenvalues which correspond to the fast and slow modes of a singularly perturbed dynamical system, ill-conditioned numerical problems are very likely to arise. However, a separation of designs, resting upon the separation of time scales, removes this liability towards ill-conditioning, and it reduces the dimensionality of the design problem as well. Furthermore, where slow and fast controllers are designed as separate entities, they might be independently implementable in different software and hardware; generally speaking, an interesting variety of design compromises ought to be flexibly entertainable. In all, a design strategy of the separate-and-composite type has great prima facie attractiveness.

\subsubsection{Reduced-Order State-Feedback Controllers}

The system-theoretic properties of Controllability and Observability, like the property of Stability, remain invariant under similarity transformations. Kokotovic and Haddad [16] showed that if both reduced-order and boundary-layer subsystems are controllable, then system (3) will 
be controllable for sufficiently small values of the parameter. A corresponding result concerning the property of Observability must, by duality, also attain. In consequence, those design techniques which are based upon the concepts of Controllability and Observability can be opened up for study in connection with the singularly perturbed system.

Assuming that both $\left(A_{0}, B_{0}\right)$ and $\left(A_{22}, B_{2}\right)$ are stabilizable, separate matrices of state feedback can independently be designed to stabilize each of the separate lower order subsystems. For the sake of specificity, we shall suppose that $\mathrm{K}_{\mathrm{s}}$ and $\mathrm{K}_{\mathrm{f}}$ are separately designed controller gains which make $\left(A_{0}+B_{0} K_{s}\right)$ and $\left(A_{22}+B_{2} K_{f}\right)$ stability matrices. We suppose, in other words, that:

$$
\overline{\mathbf{u}}=\mathrm{K}_{\mathrm{s}} \overline{\mathrm{x}} \quad \text { and } \quad \hat{\mathbf{u}}=\mathrm{K}_{\mathrm{f}} \hat{\mathbf{z}}
$$

are, respectively, an effective slow control for the reduced order model and an effective fast control for the boundary layer system. By the previously cited result of Kokotovic and Haddad, the full order system is known to be stabilizable. The question arises whether, specifically, the composite control:

$$
\mathbf{u}=\overline{\mathbf{u}}+\hat{\mathbf{u}}=\mathbf{K}_{\mathbf{s}} \overline{\mathbf{x}}+\mathrm{K}_{\mathrm{f}} \hat{\mathbf{z}}
$$

in fact stabilizes it.

In the first place, in order for this control to be concretely implementable on the actual plant, the reduced states $(\bar{x}, \hat{z})$ need to be translated into the actual states $(x, z)$. Using the approximations for $x$ and $z$ given in (9), together with the definition of $\bar{z}$ provided in (5) and the defining relations for $\overline{\mathbf{u}}$ and $\mathbf{u}$ provided by (17), the composite control (18) is expressed in terms of the plant's actual state, approximately, as:

$$
u=\left(\left(I+K_{f} A_{22}^{-1} B_{2}\right) K_{s}+K_{f} A_{22}^{-1} A_{21}\right) x+K_{f} z
$$


Under the state feedback control law (19), the plant would be governed by the state equations:

$$
\begin{gathered}
{\left[\begin{array}{c}
\dot{x} \\
e \dot{z}
\end{array}\right]=\left[\left[\begin{array}{ll}
A_{11} & A_{12} \\
A_{21} & A_{22}
\end{array}\right]+\left[\begin{array}{c}
B_{1} \\
B_{2}
\end{array}\right] K\right]\left[\begin{array}{l}
x \\
z
\end{array}\right]+\left[\begin{array}{c}
B_{1} \\
B_{2}
\end{array}\right] u} \\
K=\left[\left(I+K_{f} A_{22}^{-1} B_{2}\right) K_{s}+K_{f} A_{22}^{-1} A_{21} K_{f}\right] \equiv\left[K_{1} K_{2}\right]
\end{gathered}
$$

Note that $K_{1}$ is normally a function of both gains $K_{s}$ and $K_{f}$. However, in the special case that $A_{22}$ is already a stability matrix, the fast state feedback is not needed, and the $\mathrm{K}$ matrix takes takes the particularly simple form $\mathrm{K}=\left[\mathrm{K}_{\mathrm{s}} 0\right]$. (20) shows that the compensated plant is again singularly perturbed. A routine calculation shows that its eigenvalues are, approximately, the eigenvalues of $\left(A_{o}+B_{0} K_{\zeta}\right)$ and $1 / e$ times the eigenvalues of $\left(A_{22}+B_{2} K_{f}\right)$.

In situations where the states of the actual plant are not available for purposes of feedback control, the plant's input and output can together be made to drive a device whose output asymptotically approaches the plant's state. That is to say, an asymptotic State Observer can be employed. The full order observer is a dynamical system governed by the state equations:

$$
\left[\begin{array}{c}
\dot{\bar{x}} \\
\dot{e} \dot{\bar{z}}
\end{array}\right]=\left[\left[\begin{array}{ll}
A_{11} & A_{12} \\
A_{21} & A_{22}
\end{array}\right]-\left[\begin{array}{l}
L_{1} \\
L_{2}
\end{array}\right]\left[\begin{array}{ll}
C_{1} & C_{2}
\end{array}\right]\right]\left[\begin{array}{l}
\tilde{x} \\
\tilde{z}
\end{array}\right]+\left[\begin{array}{l}
L_{2} \\
L_{2}
\end{array}\right] y+\left[\begin{array}{l}
B_{1} \\
B_{2}
\end{array}\right] u \quad \tilde{y}=\left[\begin{array}{l}
\tilde{x} \\
\tilde{z}
\end{array}\right]
$$

The error-vector, $E \equiv\left(\tilde{x}^{t} \tilde{z}^{t}\right)^{t}-\left(x^{t} z^{t}\right)^{t}$, which represents the difference between observer output and plant state, obeys the dynamics, $\dot{\mathrm{E}}=(\mathrm{A}-\mathrm{LC}) \mathrm{E}$. For this reason, the observer gain matrix $\mathrm{L}$ must be chosen to make $(\mathrm{A}-\mathrm{LC})$ a stability matrix.

Assuming each of the lower order subsystems to be detectable, matrices $L_{s}$ and $L_{f}$ can be chosen to make both $\left(A_{0}-L_{3} C_{0}\right)$ and $\left(A_{22}-L_{5} C_{2}\right)$ stability matrices. It then follows that the gain for the full order observer, composed, by duality to (20), as: 


$$
L=\left[\begin{array}{l}
L_{s}\left(I-C_{2} A_{22}^{-1} L_{f}\right)+A_{12} A_{22}^{-1} L_{f} \\
L_{f}
\end{array}\right] \equiv\left[\begin{array}{l}
L_{1} \\
L_{2}
\end{array}\right]
$$

will place $n$ of the eigenvalues of $(A-L C)$ within $O(e)$ of the eigenvalues of $\left(A_{0}-L_{s} C_{0}\right)$, and $m$ of them within $O(e)$ of $1 / e$ times the eigenvalues of $\left(A_{22}-L_{f} C_{2}\right)$. In the special case that $A_{22}$ is already a stability matrix, only the n-dimensional observer for the slow states of the reducedorder model needs to be designed, and the $\mathrm{L}$ matrix takes the particularly simple form $L=\left[L_{s}^{t} 0^{8}\right]^{2}$.

In conclusion, if both reduced-order and boundary-layer subsystems of (3) are stabilizable by observer-based controllers, i.e. if each of these subsystems is both stabilizable and detectable, then the given plant, for sufficiently small values of the parameter, will be stabilizable through separate observer-based controllers in the separate time scales. It is known that for the observerbased controller configuration shown in Figure 4 the controller gain and the observer gain can be designed independently of one another. For the special case in which the plant is in fact a singularly perturbed system of type (3), the design of the observer-based controller thus reduces to four independent lower-order eigenvalue-placement problems.

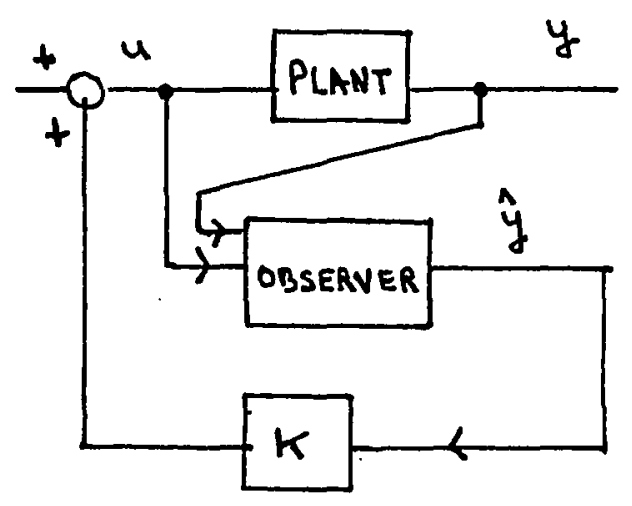

Figure 4 


\subsubsection{H. K. Khalil's Reduced-Order Dynamic Controller}

An important paper by H.K. Khalil, "On the Robustness of Output Feedback Control Methods to Modelling Errors" [14], broaches the subject of dynamic output feedback compensation for the singularly perturbed plant. This paper restricts consideration to cases in which the reduced-order dynamic controller designed for the "slow" subsystem is by itself capable of achieving the requisite regulation in a given situation. Khalil has no need for a "fast" boundarylayer dynamic controller in his chosen application, because he assumes the fast dynamics of the given plant to be open-loop stable.

Khalil has in mind the situation in which singular-perturbational analysis is used as a tool to analyze the robustness of controller design. Evidently, real plants are never perfectly modelled; still, it is a practical need that the controllers designed for these plants be robust with respect to expectable modelling errors. In this light, it is convenient to allow a deliberately simplified model of the plant to be used for purposes of engineering analysis and design, regarding the "real" plant as a singular perturbation off this simplified model. The design model is then, formally, the "real" plant's reduced-order slow subsystem. The fast boundary-layer subsystem, meanwhile, then formally represents the extent of the real plant's deviation from the design model, with perturbation parameter then in effect measuring the mismatch between them in terms of a specific set of structured uncertainties. This application has precisely the structure of the following problem, which Khalil undertakes to solve.

Khalil presents his dynamic compensator in the state-space form:

$$
\begin{gathered}
\dot{v}=G_{1} v+G_{2} y+G_{3} u \\
u=F_{1} v+F_{2} y
\end{gathered}
$$

The resultant plant-compensator configuration is presented in the block-diagram of Figure 5: 


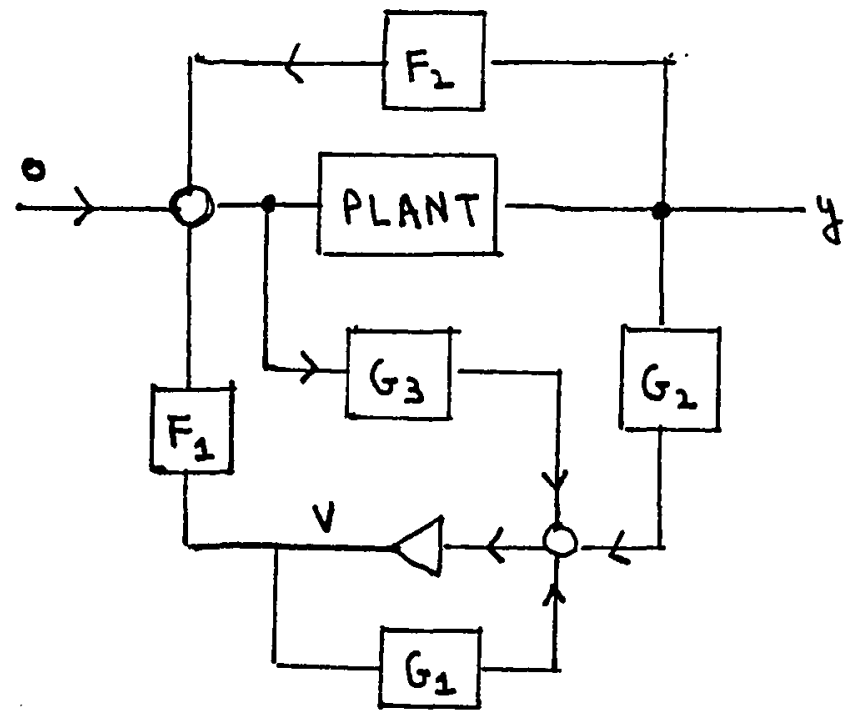

Figure 5

As a model for the dynamic compensator, (23) is extremely general. Clearly, it covers the case of dynamic output compensation in the plant's feedback path, inclusive of constant-gain static output feedback as one limiting special case. This model is sufficiently versatile to cover as well the case of linear state feedback and the observer-controller configuration, as will subsequently be demonstrated.

The closed-loop model to the designer refers to the configuration in which controller (23) is applied to reduced-order subsystem (5). Khalil shows that this configuration has the state-space description:

$$
\left[\begin{array}{l}
\dot{x} \\
\dot{v}
\end{array}\right]=\left[\begin{array}{ll}
A_{0}+B_{0}\left(I-F_{2} D_{0}\right)^{-1} F_{2} C_{0} & B_{0}\left(I-F_{2} D_{0}\right)^{-1} F_{1} \\
\left(G_{2}\left(G_{3}+G_{2} D_{0}\right)^{-1} F_{2}\right) C_{0} & G_{1}+\left(G_{3}+G_{2} D_{0}\right)\left(I-F_{2} D_{0}\right)^{-1} F_{1}
\end{array}\right]\left[\begin{array}{l}
x \\
v
\end{array}\right] \equiv\left[\begin{array}{ll}
W_{11} & W_{12} \\
W_{21} & W_{22}
\end{array}\right]\left[\begin{array}{l}
x \\
v
\end{array}\right]
$$

Evidently, $F_{2}$ needs to be chosen consistently with keeping $\left(I-F_{2} D_{0}\right)$ nonsingular. It is further assumed that $G_{1}, G_{2}, G_{3}, F_{1}$ and $F_{2}$ have been chosen in a way that makes $W$ a stability matrix, since controller (23) is designed precisely for its capability to stabilize and regulate system (5). 
The actual closed-loop system, referring to the configuration in which controller (23) is applied to the singularly perturbed system which represents the actual plant, i.e. to system (3), needs also to be conceptualized. Khalil shows this actual closed-loop system to have the statespace description:

$$
\left[\begin{array}{c}
\dot{x} \\
\dot{v} \\
\dot{e} z
\end{array}\right]=\left[\begin{array}{lll}
A_{11}+B_{1} F_{2} C_{1} & B_{1} F_{1} & A_{12}+B_{1} F_{2} C_{2} \\
\left(G_{2}+G_{3} F_{2}\right) C_{1} & \left(G_{1}+G_{3} F_{1}\right) & \left(G_{2}+G_{3} F_{2}\right) C_{2} \\
A_{21}+B_{2} F_{2} C_{1} & B_{2} F_{1} & A_{22}+B_{2} F_{2} C_{2}
\end{array}\right]\left[\begin{array}{l}
x \\
v \\
z
\end{array}\right] \equiv\left[\begin{array}{ll}
H_{1} & H_{2} \\
\\
H_{3}
\end{array}\right]\left[\begin{array}{l}
x \\
v \\
v \\
z
\end{array}\right]
$$

The actual closed-loop system is evidently itself again a singularly perturbed system, whereas the closed-loop model to the designer is not in itself singularly perturbed.

Khalil establishes by computation that:

$$
\mathrm{H}_{4}^{-1}=\mathrm{A}_{22}^{-1}\left(\mathrm{I}-\mathrm{B}_{2}\left(\mathrm{I}-\mathrm{F}_{2} \mathrm{D}_{0}\right)^{-1} \mathrm{~F}_{2} \mathrm{C}_{2} \mathrm{~A}_{22}^{-1}\right)
$$

$H_{4}$ is nonsingular, since by assumption both $A_{22}$ and $\left(I-F_{2} D_{0}\right)$ are nonsingular. Next, $H_{0}$ is defined:

$$
\mathrm{H}_{0} \equiv \mathrm{H}_{1}-\mathrm{H}_{2} \mathrm{H}_{4}^{-1} \mathrm{H}_{3}
$$

By further computation, Khalil establishes that $\mathrm{H}_{0}$ equals the matrix $\mathrm{W}$, defined in (24). That is to say, system (24) is in fact the reduced-order slow approximating model for system (25). By Theorem 1, the eigenvalues of $\mathrm{H}$ are then, approximately: the eigenvalues of $\mathrm{H}_{0}$ plus $1 / \mathrm{e}$ times the eigenvalues of $\mathrm{H}_{4} \cdot \mathrm{H}_{0}$, being identically the matrix $\mathrm{W}$, is already by design a stability matrix. Therefore, for stability of the actual closed-loop system, only one further condition remains: $\mathrm{H}_{4}$ needs as well to be a stability matrix. As previously mentioned, Khalil assumes the fast dynamics or "high-frequency parasitics" of the plant to be stable. That is to say, he takes it as a hypothesis that $A_{22}$ is a stability matrix. Since by the above reasoning $H_{4}$, or $\left(A_{22}+B_{2} F_{2} C_{2}\right)$, is 
required to be a stability matrix, Khalil concludes his paper with the identification of a number of special cases in which the stability of $A_{22}$ sufficiently guarantees the stability of $\mathrm{H}_{4}$.

In the first such special case, the constitution of the given plant is such that either $B_{2}=0$ or $C_{2}=0$-- i.e. the fast modes are either not directly actuated from the input or not directly sensed at the output. Interestingly, the case of linear state feedback for a singularly perturbed plant with already-stable fast dynamics is assimilated to the paradign of (23), with: $C_{1}=I, C_{2}, G_{1}, G_{2}, G_{3}$ and $F_{1}$ all null matrices, and $F_{2}=K_{3}$. In consequence, $\left(A_{0}+B_{0} K_{s}\right)^{\prime} s$ being a stability matrix is sufficient in this case to guarantee stability of the actual plant under the feedback law $\mathrm{u}=\mathrm{K}_{\mathrm{s}} \mathrm{x}$ for all suitably small departures from the design model. A "robustness" property is thus enjoyed by the linear state feedback controller design on singularly perturbed plants with open-loop-stable high-frequency dynamics.

A second special case, the case of $F_{2}=0$, i.e. the case of no static feedback from the output to the input of the plant, likewise guarantees the stability of $\mathrm{H}_{4}$ from the stability of $\mathrm{A}_{22}$. Interestingly, the case of state feedback based upon an observer for the reduced-order subsystem of a singularly perturbed system with open-loop-stable fast dynamics-assimilates to the paradigm controller (23), with: $F_{2}=0, F_{1}=K_{3}, G_{1}=A_{0}-L_{3} C_{0}, G_{2}=B_{0}-L_{3} D_{0}$, and $G_{3}=L_{s}$. Assuming that the gain matrices $\mathrm{K}_{s}$ and $\mathrm{L}_{\mathrm{s}}$ have been properly chosen, the robustness property of the observerless state feedback again holds in connection with the present class of systems.

In the case of static output feedback, $F_{1}, G_{1}, G_{2}$, and $G_{3}$ are null, while $F_{2}$ is equal to some nonzero constant matrix. The robustness property is not guaranteed for this case. Since anything that can be done with constant gain feedback from the output can as well be done with state feedback but not vice versa, the static output feedback design is in general less versatile than the design involving linear state feedback which was previously discussed. 
The issue of constant gain output feedback for the singularly perturbed system is treated in detail in a paper by Fossard and Magni [7]. These authors' innovation is to allow the output vector to be partitioned into two subvectors, i.e:

$$
y=\left[\begin{array}{l}
y_{1} \\
y_{2}
\end{array}\right]=\left[\begin{array}{ll}
C_{11} & C_{12} \\
C_{21} & C_{22}
\end{array}\right]\left[\begin{array}{l}
x \\
z
\end{array}\right] \equiv\left[\begin{array}{ll}
C_{1} & C_{2}
\end{array}\right]\left[\begin{array}{l}
x \\
z
\end{array}\right]
$$

Fossard and Magni hypothesize that stabilizing static output controllers have first been designed separately for the slow and fast subsystems. They hypothesize, specificically, that a $G_{s}$ that makes $\left(A_{0}+B_{0} G_{s} C_{0}\right)$ stable yields the slow control law $\bar{u}=G_{s} \bar{y}$, while a $G_{f}$ that makes $\left(A_{22}+B_{2} G_{f} C_{2}\right)$ stable yields the fast control law $\hat{u}=G_{f} \hat{y}$. These authors then address the question: under what conditions on the Output Matrix can the composite control $u=G_{s} \bar{y}+G_{f} \hat{y}$ be composed as $u=G_{1} y_{1}+G_{2} y_{2}$, for some $G_{1}$ and $G_{2}$ which may be functions of $G_{s}, G_{f}$ and any of the relevant system submatrices? That is to say, under what conditions can the full control be composed in terms of the actual output subvectors $y_{1}$ and $y_{2}$ that are hypothesized to available ? Recall that (from (5)) $\bar{y}=C_{0} \bar{x}+D_{0} \bar{u}$ and that (from (8)) $\hat{y}=C_{2} \hat{z}$, while $y_{1}$ and $y_{2}$ are defined (by (28)) in terms of the "true" states $\mathrm{x}$ and $\mathrm{z}$.

Through different ways of partitioning Output vector and Output matrix -- in particular, through the exploitation of a number of identifiable special cases (e.g. $\mathrm{C}_{22}=0$ ) which conduce to particularly favorable applications in connection with the structure of systems (3), (5) and (8) -Fossard and Magni succeed in enhancing the versatility of the static output concept for the singularly perturbed system. But Khalil's robustness property cannot be guaranteed for the static output feedback, even in the most favorable of these special cases. Khalil [15] in fact provides an interesting example in which static output feedback for a design model itself without static feedback from output to input nonetheless destabilizes the actual plant for any nonzero value of the perturbation parameter within some neighborhood of $e=0$. Apparently, the high-frequency parasi- 
tics, even where they are open-loop-stable, have nonetheless the potential under feedback to destabilize the closed-loop system, unless a design method which guarantees a degree of robustness against singular perturbations has been employed.

\subsection{OPERATOR-THEORETIC ANALYSIS OF THE SINGULARLY PERTURBED SYSTEM}

\subsubsection{Mathematical Characterization of the Implicit Singularly Perturbed System}

With the paper, "Hierarchical Aggregation of Linear Systems in Multiple Time Scales", by Coderch, Willsky, Sastry and Castanon [3], discussion moves on to the general class of implicit singularly perturbed systems. Coderch et al study an e-dependent linear autonomous system which is more general than system (3), namely the system:

$$
\dot{x}_{e}(t)=A(e) x_{e}(t) \quad x_{e}(0)=x_{0}
$$

In (29) $A(e)$ is restricted only to being analytic as a matrix function of e. In other words, each term of $\mathrm{A}(\mathrm{e})$ is assumed to have a convergent power series expansion in $\mathrm{e}$ about $\mathrm{e}=0$, valid for all $e$ in $\left(0, e^{*}\right]$ for some upper bound $e^{*}$. Consequently, the eigenvalues of $A(e)$, namely the zeros of the polynomial in s with e-dependent coefficients, $\operatorname{det}(\mathrm{sI}-\mathrm{A}(\mathrm{e})$ ), will be analytic functions of e. As before, e is understood to be a small positive scalar parameter. Therefore, system (29) may be thought of as having been perturbed off the system:

$$
\dot{x}_{0}(t)=A(0) x_{0}(t) \quad x_{0}(0)=x_{0}
$$

System (29) is regarded as a singularly, rather than regularly, perturbed version of system (30) -- or, in itself, as an implicitly singularly perturbed system -- if it has a constant number of nonzero eigenvalues for all values of $\mathrm{e}$ in $\left(0, \mathrm{e}^{*}\right)$ for some bound $\mathrm{e}^{*}$, but only a strictly 
smaller number of them at the value $e=0$. Thus, in the implicitly singularly perturbed system, $A(0)$ is singular, and the rank of $A(0)$ is strictly less than the normal rank (or "nrank") of $A(e)$.

The small parameter does not necessarily multiply derivatives in (29), as it does in the system which is explicitly singularly perturbed; consequently, the foregoing analysis of system (3) will not suffice as an analysis of the more general system that is presently under study. Coderch et al do not attempt to transform the more general system into explicit singularly perturbed form, in order to invite the techniques of the foregoing discussion to be applicable to it. Rather, they seek (i) an intrinsic time-domain criterion which identifies those implicit singularly perturbed systems with the multiple-time-scale property, and (ii) a method. aopropriately related to the criterion, for decomposing these systems into their sets of lower-order approximating subsystems on the appropriate time scales.

We first look at the following explicitly singularly perturbed system, which displays multiple (i.e. more than two) time scale behavior:

$$
\left[\begin{array}{l}
\dot{x}_{1} \\
e \dot{x}_{2} \\
\ldots . . \\
e^{n-1} \dot{x}_{n n}
\end{array}\right]=\left[\begin{array}{cccc}
A_{11} & A_{12} & \ldots . . & A_{1 n} \\
A_{21} & A_{22} & \ldots . . & A_{2 n} \\
\ldots . . & \ldots . . & \ldots . . & \ldots . . \\
A_{n 1} & A_{n 2} & \ldots . . & A_{n n}
\end{array}\right]\left[\begin{array}{l}
x_{1} \\
x_{2} \\
. \\
x_{n n}
\end{array}\right]
$$

By a renormalization of the time variable, $A(e)$ in (31) can be cast into the form of an analytic efunction. In the sequence of time variables:

$$
T_{j}=e^{j-1} t \quad(j=1, \ldots, n)
$$

the fastest time-scale is the $T_{1}-$ or t-time scale, and the slowest is the $T_{n}$ - time-scale. In this slowest time-scale, system ( 31$)$ is described by: 


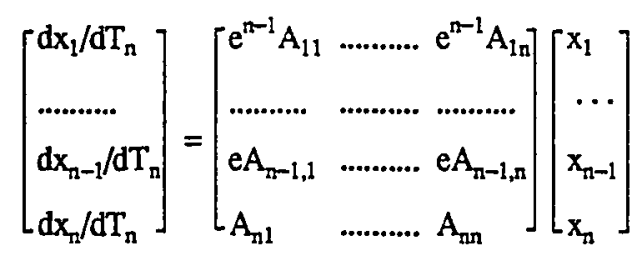

Note that in (33), the matrix function $\mathrm{A}(\mathrm{e})$ has the simple finite expansion:

$$
A(e)=A_{0}+e A_{1}+\ldots . .+e^{n-1} A_{n-1}
$$

By extrapolation from the behavior of the two-time-scale explicitly singularly perturbed system which has already been analyzed, the behavior of system (33) must be characterizable as follows. When $t$ is $0(1)$, the first and fastest set of dynamics is evolving normally. $T_{j}$ being at this time $O\left(e^{j-1}\right)$, all the later, slower sets of dynamics (those which normally evolve in time-scales with $\mathrm{j}$ strictly greater than 1 ) have not yet effectively begun to evolve. When thas reached $0(1 / \mathrm{e})$, $T_{2}$ reaches $0(1)$, and the second set of dynamics normally unfold. At this point in time, the first set of dynamics have (assuming stability) already come to equillibrium; but, $T_{j}$ now being $0\left(e^{j-2}\right)$, the third and higher sets of dynamics have still not effectively commenced their evolutions. When $t$ reaches $0\left(1 / e^{n-1}\right), T_{n}$ is $0(1)$, and the slowest dynamics finally evolve, all faster dynamics having (assuming stability) already come to equillibrium. From the spectral point of view, $A(e)$ is known to have $\mathrm{n}$ distinct groups of eigenvalues. The dominant behavior of the system on the $T_{j}$-time-scale is determined by the eigenvalues of order $O\left(e^{j}\right)$. Clearly, the explicit singularly perturbed system of type (31) displays multiple-time-scale behavior.

We now retum to the characterization of multiple-time-scale behavior for the implicitly singularly perturbed system of type (29). Since A(e) in (29) is a matrix analytic function, it can be expanded in the convergent power series: 


$$
A(e)=\sum_{j=0}^{\infty} e^{j} A_{j}
$$

Consequently, the trajectories of the (homogeneous) system of type (29) are described by:

$$
x_{c}(t)=\exp \left(\sum_{j=0}^{\infty} A_{j} e^{j} t\right) x_{0}
$$

By the previous discussion, trajectories of the type:

$$
x(t)=\exp \left(\sum_{j=0}^{m} A_{j} e^{j} t\right) x_{0}
$$

are known to display multiple-time-scale behavior. If it can be shown that a set of trajectories of type (36), derived from a given implicitly singularly perturbed system of type (29), are sufficiently well approximable by a set of trajectories like (37) (which derive from an explicit singularly perturbed system of type (33) or type (31)), it will then be deemed that the corresponding system of type (29) is itself multiple-time-scale.

\subsubsection{Perturbation Theory for Linear Operators}

Coderch's detailed analysis rests upon a rather complicated Operator Theory -- namely, the Perturbation Theory for Linear Operators which is found in the work of T. Kato [11]. Since this Operator Theory is not needed for any of the developments in the following Chapters, it is here reviewed in no greater detail than suffices for tracing one basic result concerning the implicitly singularly perturbed multiple-time-scale systems.

Suppose that A is a linear operator on $C^{n}$. Of Operator Theoretic interest are: the spectral decomposition of $\mathrm{A}$ in terms of eigenprojection and eigennilpotent matrices corresponding to the 
eigenvalues of $A$, the resolvent operator corresponding to the operator $A$, and the Laurent Series expansion of the resolvent operator about the eigenvalues of $\mathrm{A}$. The operator $\mathrm{A}$ is defined to have Semisimple Null Structure if the eigennilpotent corresponding to its zero-eigenvalue is a zero matrix. Equivalently, A has Semisimple Null Structure if it has a complete set of eigenvectors corresponding to its zero-eigenvalues. When A has Semisimple Null Structure, it follows that both the spectral decomposition of $\mathrm{A}$ and the Laurent expansion of the resolvent of $\mathrm{A}$ about the zero-eigenvalue take peculiarly simple forms.

The operator $A$ is next allowed to be perturbed into the operator $A(e)$, which is assumed to be expandable in the form of (35). By Kato's Theory, corresponding expressions for the perturbed eigenvalues, the perturbed resolvent operator and the perturbed eigenprojection matrices are also obtainable. The eigenvalues of $A(e)$, being the zeros of det (sI - A(e)), will be analytic functions of e. By the hypothesis that $A(e)$ is the matrix of State of a given system of type (29) which is implicitly singularly perturbed, the number of its nonzero eigenvalues will be constant throughout $\left(0, e^{*}\right)$ for some bound $e^{*}$, but at $e=0$ their number will be strictly smaller. The perturbed operator A(e) will thus have a group of near-zero eigenvalues which "split off" from the zero-eigenvalues of $\mathrm{A}(0)$. In the following development, $\mathrm{P}(\mathrm{e})$ will designate the total eigenprojection of $A(e)$ corresponding to this group of near-zero eigenvalues.

\subsubsection{Algorithm for Determining the Time-Scale Hierarchies}

At the point in their exposition at which an iterative algorithm is about to be submitted for determining the time-scale hierarchies (if they exist) of the implicit singularly perturbed system, Coderch et al switch to the double-subscript notation:

$$
A_{k}(e)=A_{k 0}+\sum_{j=1}^{\infty} e^{j} A_{k j}
$$


In (38), the first subscript of the pair designates the iteration-round, while the second subscript has the sense which the lone subscript had in (35). To start off the algorithm, $A(e)$ is re-named $A_{0}(e)$ and is expanded according to (38). Examination next focusses on the term $A_{00}$, the first term in the expansion. If $A_{00}$ is found to have Semisimple Null Structure, then $A_{1}(e)$ is presented as:

$$
A_{1}(e)=\left(P_{0}(e) A_{0}(e)\right) / e
$$

$A_{1}(e)$ in turn is expanded according to (38), and next $A_{10}$ is examined. If $A_{10}$ has Semisimple Null Structure, then $\mathrm{A}_{2}(\mathrm{e})$ is presented as:

$$
A_{2}(e)=\left(P_{1}(e) A_{1}(e)\right) / e=\left(P_{1}(e) P_{0}(e) A_{0}(e)\right) / e^{2}
$$

Next, $A_{2}(e)$ is expanded, and $A_{20}$ is examined for Semisimple Null Structure. The sequence is guaranteed always to terminate at some finite step. It will terminate in one of two ways. The algorithm stops with $A_{m}(e)$, if either (a) $A_{m o}$ fails to have Semisimple Null Structure or (b) $A_{m+1}$ is the zero operator.

By definition, $A(e)$ has Multiple Semisimple Null Structure if each of the $A_{k o}$ 's defined in the constructive procedure has Semisimple Null Structure. A(e) will have Multiple Semisimple Null Structure if and only if the algorithmic procedure terminates through condition (b) rather than through condition (a). Equivalently, in such a case: $\sum_{k=0}^{m} \operatorname{rank}\left(A_{k o}\right)=n \operatorname{nrank}(A(e))-$ i.e., the normal rank of $A(e)$ is "filled" by the series of approximating first-order parameter-independent matrices which derive from the algorithmic procedure. An A(e) which has Multiple Semisimple Null Structure is further defined to be Multiply Semistable if all the nonzero eigenvalues of all the $A_{k o}$ 's are open left-half-plane. 
The highpoint of the Coderch paper is the proof that A(e)'s being Muliply Semistable is an equivalent condition for system (29)'s possessing the multiple-time-scale property. When a system of type (29) is in fact Multiply Semistable, Coderch et al show that:

$$
\limsup _{e \downarrow 0} \sup _{t \geq 0}\left\|\exp (A(e) t)-\exp \left(\sum_{k=0}^{m} A_{k 0} e^{k} t\right)\right\|=O(e)
$$

In (41) "II II" may be any of the equivalent matrix norms on $C^{n \times n} . \operatorname{Exp}\left(\sum_{k=0}^{m} A_{k 0} e^{k} t\right)$ is now a uniform (i.e. $\sup _{\geq \geq 0}$ ) asymptotic (i.e. $\lim _{e \downarrow 0}$ ) approximation to $\exp (\mathrm{A}(\mathrm{e}) \mathrm{t})$. In consequence of $(41)$, the trajectories of the Multiply Semistable system of type (29) are successfully approximable by those of a system of type (31), and so the system in question is deemed itself to display multiple-time-scale behavior. The e-independent $A_{k o}$ matrices which are yielded by the algorithmic procedure are, furthermore, themselves the reduced-order models of $A(e)$ that correspond to the $m+1$ time-scales which suffice for approximating the full evolution of system (29)'s time dynamics. The partial time-scale decompositions corresponding to these reduced-order models have validity because, for $\mathrm{k}=0,1, \ldots, \mathrm{m}-1$, it holds that:

$$
\lim _{e \downarrow 0} \sup _{0<t<\infty}\left\|\exp \left(A(e)\left(t / e^{k}\right)\right)-P_{0} \ldots . . P_{k-1} \exp \left(A_{k 0} t\right)\right\|=O(e)
$$

In their paper, Coderch, Willsky, Sastry and Castanon have succeeded in giving a highly general time-domain result for the multiple-time-scale systems. In the next chapter, the work of D. W. Luse [18-21] will be shown to provide a frequency-domain virtual counterpart to this highly significant result. It is the frequency-domain rather than the time-domain formulation which opens the door to an analysis of the multiple-time-scale system from the standpoint of modem Algebraic Systems Theory. 


\section{CHAPTER II}

\section{FREQUENCY DOMAIN ANALYSIS OF THE MULTIPLE-TIME-SCALE SYSTEMS}

\subsection{REVIEW OF BASIC CONCEPTS IN THE ALGEBRAIC THEORY OF LINEAR DYNAMICAL SYSTEMS}

Before presenting D. W. Luse's work [18-21] on the frequency-domain characterization of the multiple-time-scale system, we review some basic concepts and techniques from the modem Algebraic Theory of multivariable linear dynamical systems. The transfer matrix of a multivariable lumped linear time-invariant system has the form of a rational matrix, $G(\mathrm{~s})$. The review therefore focusses on the deep algebraic structure of rational matrices. Two methods for revealing this deep structure are studied: (A) the Coprime Factorization of G(s) over Polynomial Matrices and (B) the conversion to the canonical McMillan Form of G(s). Whereas the Algebraic Theory derives, basically, from the innovative work of Rosenbrock [27], the present review also borrows some of its formuiations from more recent treatments of the subject by Kailath [10] and Chen [2], which enable the clarity and the economy of the exposition to be enhanced. In the following discussion, all transfer matrices are assumed to be proper, i.e. $g_{i j}(\infty)<\infty$ for each entry $g_{i j}(s)$ of the matrix $G(s)$. 


\subsubsection{Coprime Factorization over Polynomial Matrices of the Rational Transfer Matrix, G(s)}

Let $\mathbf{R}[\mathbf{s}]$ denote the Ring of polynomials in the indeterminate s over the Real Field. It is well known in Algebra that the Ring $R[s]$ is in fact a Principal Ideal Domain (PID). $R[s]$ is as well, with the usual degree function, $\operatorname{deg}(f)=$ degree of the polynomial $f(s)$, an Euclidean Domain. Because it is a Ring and not a Field, not all its nonzero elements are invertible, nor can the arbitrary element be divided by another arbitrary nonzero element with their quotient necessarily still defined as an element in R[s]. In an Euclidean Domain, the invertible elements, or units, are the elements of degree zero. For $\mathrm{R}[\mathrm{s}]$, these units are the nonzero constant polynomials.

By definition, $f$ is a divisor of $g$ in the Ring $R$ if there exists an $h$ in $R$ such that $g=f$. $h$ is defined to be a Greatest Common Divisor of $f$ and $g$ (abbrev: $G C D(f, g)$ ) if (i) $h$ is a common divisor of $f$ and $g$ and (ii) every other common divisor is in fact a divisor of $h$. (f,g) are defined to be a coprime pair if their GCD is a unit of $R$. In a Principal Ideal Domain a GCD of any two elements is guaranteed to exist and to be unique to within multiplication by units. In the Ring $R[s]$, if the GCD is required to be monic, then the GCD can in fact be uniquely specified. Furthermore, if $h$ is a GCD(f,g), the existence is guaranteed of elements $p$ and $q$ in the PID to satisfy the equation: $h=p f+q g$.

Let $\mathbf{R}(\mathbf{s})$ denote the Field of rational functions in the indeterminate $s$ with real coefficients. It is well known in Algebra that the Field of Quotients of $R[s]$, the smallest extension of $R[s]$ in which every nonzero element is invertible (as well, the smallest Field which includes $R[s]$ ), is $R(s)$. Generally speaking, when $F$ is the Field of Quotients of $R$ and $R$ is a Principal Ideal Domain, it is the case not only (i) that the quotient of any two elements in $R$ (provided denominator element is nonzero) will in fact be defined as an element of $F$, but also (ii) that every element of $\mathrm{F}$ can be factored as the quotient of two elements in $\mathrm{R}$ which are coprime in $R$. In the case where $R$ is $R[s]$ and $F$ is $R(s)$, condition (ii) gives the familiar "reduced-form" of fractions of 
polynomials. Technically, the reduction of a fraction involves the extraction of a GCD from numerator and denominator.

We now let $\mathbf{M}(\mathbf{R}[s])$ stand for the Ring of polynomial matrices, i.e. matrices whose elements are polynomials, and $\mathbf{M}(\mathbf{R}(\mathbf{s}))$ for the Ring of rational matrices, i.e. matrices whose elements are rational functions. The units of $M(R[s])$, called the unimodular matrices, are by definition those square polynomial matrices which have inverses in $M(R[s])$. A very useful equivalence is that $A$ in $M(R[s])$ is unimodular if and only if $\operatorname{det}(A)$ is a unit of $R[s]$, a nonzero constant polynomial. The units of $M(R(s))$ are the wider class of nonsingular matrices. Since $R(s)$ has a Field structure, every nonzero element of $R(s)$ is a unit. Consequently, every $A$ in $M(R(s))$ for which $\operatorname{det}(A) \neq 0$ has an inverse in $M(R(s))$. It is apparent that matrices which are unimodular in $M(R[s])$ will be nonsingular when considered as elements of $M(R(s))$. It is also evident that a nonsingular matrix $A$ in $M(R(s))$ will be nonsingular over the Complex Field for all complex values of $s$ except those $s_{i}$ which are zeros of $\operatorname{det}(\mathrm{A}(\mathrm{s}))$.

Due to the non-commutativity of the multiplication operation in the matrix case, left- and right- forms must be distinguished corresponding to those properties which have already been defined for scalar Rings. For matrices, if $A=B C$, we say that $C$ is a right-divisor of $A$ and that $A$ is a left-multiple of $C$. Now, if $N(s)$ and $D(s)$ are polynomical matrices with the same number of columns, and if $\mathrm{Q}(\mathrm{s})$ is a given polynomial matrix of the appropriate square dimension, we say that $\mathrm{Q}(\mathrm{s})$ is a Greatest Common Right Divisor (abbrev: GCDR) of $\mathrm{N}$ and D, if: (i) $\mathrm{Q}$ is a common right divisor of $\mathrm{N}$ and $\mathrm{D}$, and (ii) $\mathrm{Q}$ is a left-multiple of any other common right divisor. (N,D) are by definition Right-Coprime if any GCDR(N,D) is a unimodular matrix. Also, if $Q$ is a $\operatorname{GCDR}(\mathrm{N}, \mathrm{D})$, there will exist polynomial matrices $\mathrm{X}(\mathrm{s})$ and $\mathrm{Y}(\mathrm{s})$ such that: $\mathrm{Q}=\mathrm{XD}+\mathrm{YN}$. Here, the GCDR is unique to within multiplication by unimodular matrices. Consequently, when (N,D) are right-coprime, there will exist elements $\mathrm{X}$ and $\mathrm{Y}$ to satisfy the right Bezout Equation, $\mathrm{XD}+\mathrm{YN}=\mathrm{I}$. For GCDL and left-coprimeness, entirely analogous dual definitions are 
stipulable.

To achieve a more intuitive feeling for the coprimeness of polynomial matrices, consider that $(N, D)$ are right-coprime if and only if there do not exist polynomial matrices $A(s)$ and $B(s)$, with $A$ nonsingular and $\operatorname{deg}(\operatorname{det}(A(s)))$ strictly less that $\operatorname{deg}(\operatorname{det}(D(s)))$, to satisfy the relation: $A^{-1} B=N D^{-1}$. This compares with the more familiar statement for the scalar case: polynomials $d(s)$ and $n(s)$ are coprime if and only if there do not exist polynomials $a(s)$ and $b(s)$, with $a(s)$ nonzero and $\operatorname{deg}(a(s))$ strictly less than $\operatorname{deg}(d(s))$, such that: $b(s) / a(s)=n(s) / d(s)$-- i.e. the fraction $n(s) / d(s)$ cannot be any further "reduced".

An extremely important property which follows from the fact that $R(s)$ is the field of quotients of $R[s]$ is that: the arbitrary $G$ in $M(R(s))$ can be factored as a matrix fraction over polynomial matrices of the form $G=N D^{-1}$ for some right-coprime pair (N,D) in $M(R[s])$. Equally, it can be factored as a matrix fraction of the form $G=\bar{D}^{-1} \bar{N}$ for some left-coprime pair $(\bar{D}, \bar{N})$. This property is so basic to the Fractional Representation Approach that it has in effect given this approach its name! $D$ ( or $\bar{D}$ ) must of course be nonsingular, but it will fail to be unimodular if $G(s)$ belongs to the set-difference, $M(R(s))-M(R[s])$.

When given an arbitrary, i.e. not necessarily coprime, matrix-fraction description: $G=N_{1} D_{1}^{-1}$, the coprime factorization can be obtained from it as follows. Suppose that $Q_{1}$ is a $\operatorname{GCDR}\left(N_{1}, D_{1}\right)$. It holds that $Q_{1}$ will be nonsingular if $D_{1}$ is nonsingular. Then, in terms of $D_{0} \equiv D_{1} Q_{1}^{-1}$ and $N_{0} \equiv N_{1} Q_{1}^{-1}$, the right-coprime matrix fraction description, $G=N_{0} D_{0}^{-1}$, is obtained. This procedure shows the matrix meaning of "reduced-form fraction". 


\subsubsection{McMillan Form of $\mathrm{G}(\mathrm{s})$}

Two matrices over a given Ring are said to be equivalent if they can be related by unimodular matrices; that is to say, $A$ is equivalent to $B$ if and only if $A=U B V$ for some unimodular $U$ and V. It is well known in Algebra that any matrix over a Principal Ideal Domain is equivalent to a matrix in the canonical Smith Form:

$$
S(s)=\operatorname{diag}\left(h_{1}(s), h_{2}(s), \ldots, h_{r}(s)\right)
$$

In (1), the product $\left(h_{1} h_{2} \cdots h_{j}\right)$ represents the GCD of all $j \times j$ minors of $A$. Thus:

$$
h_{j}=\frac{G C D(\text { all j } \times j \text { minors })}{G C D(\text { all } 1 \times 1 \text { minors }) \times C D(\text { all } 2 \times 2 \text { minors }) \circ \cdots G C D(\text { all }(j-1) \times(j-1) \text { minors })}
$$

It is assumed in (1), for the sake of simplicity, that $\mathrm{A}$ is of square dimension $\mathrm{r} \times \mathrm{r}$ and has full rank. Clearly, $h_{j}$ divides $h_{j+1}$ for all the appropriate $j$ 's. The $h_{j}^{\prime} s$ are called the invariant factors of $A$ and are unique to within multiplication by units of the PID. When the PID is, specifically, $\mathrm{R}[\mathrm{s}]$, these invariant factors can be uniquely specified by requiring them to be monic. For any $R C F, G(s)=N(s) D(s)^{-1}$, and for any $L C F, G(s)=\bar{D}(s)^{-1} \bar{N}(s), N$ and $\bar{N}$ are equivalent and therefore have the same Smith Form; while $D$ and $\bar{D}$, if not having the same Smith Form (for they may be of different dimension), must still have the same invariant factors.

For a matrix over the Field of Fractions of the PID, a canonical McMillan Form is defined. Specifically, for the matrix $G(s)$ in $M(R(s))$, this form can be obtained through the following procedure. First, $G(s)$ is written as $G(s)=(d(s) I)^{-1} A(s)$, where $d(s)$ is the monic least common denominator of all the elements of $G(s)$. Then, the Smith Form of $A(s)$ is obtained and represented by $S(s)$. Finally, $(d(s) I)^{-1} S(s)$ is formed, with all polynomial factors common to numerator and denominator of any single entry cancelled, to render the resulting fractions 
coprime over R[s]. This procedure yields the McMillan Form:

$$
M(s)=\operatorname{diag}\left(b_{j}(s) / a_{j}(s)\right) \quad j=i, \ldots, r
$$

In (3) it is again assumed, for simplicity, that the matrix is of square dimension $\mathrm{r} \times \mathrm{r}$ and has full rank. In the McMillan form, $b_{j}$ divides $b_{j+1}$ and $a_{j+1}$ divides $a_{j}$ for all the relevant $j$ 's. As well, $a_{1}(s)=d(s)$

\subsubsection{Poles and Zeros of $\mathrm{G}(\mathrm{s})$}

Either (A) the Coprime Factorization over Polynomial Matrices or (B) the McMillan Form can now be used to reveal the deep algebraic structure of $G(s)$. A pole of $G(s)$ is defined as either: (A) a zero of $\operatorname{det}(\mathrm{D}(\mathrm{s}))$, where $\mathrm{D}(\mathrm{s})$ is any right- of left- matrix-denominator in a coprime factorization of $G(s)$, or (B) a zero of any of the $a_{j}(s)$ denominator-polynomials in the McMillan form of $G(s)$. A transmission-zero is defined either by: (A) a value of the complex variable $s$ for which $\mathrm{N}(\mathrm{s})$ loses full rank, where $\mathrm{N}(\mathrm{s})$ is any right- or left- matrix-numerator in a coprime factorization of $G(s)$, or $(B)$ a zero of any of the $b_{j}(s)$ numerator-polynomials in the McMillan Form of $G(s)$. Any value $s_{1}$ which causes $N(s)$ to lose full rank must be a zero of one of the invariant factors of $\mathrm{N}(\mathrm{s})$; in the special case that $\mathrm{G}(\mathrm{s})$ is square, this $\mathrm{s}_{1}$ is as well a zero of $\operatorname{det}(\mathrm{N}(\mathrm{s})$ ).

The above definitions entail the following algebraic properties for the poles and transmission-zeros of the MIMO transfer function. Every pole of $\mathrm{G}(\mathrm{s})$ must be a pole of some element of $G(s)$, and any pole of any element of $G(s)$ will be a pole of $G(s)$ as well. In particular, if $\mathrm{G}$ is proper, $\mathrm{G}$ cannot have a pole at infinity. The poles of MIMO transfer functions behave similarly to their SISO counterparts in this respect. The algebraic behavior of the MIMO transmission-zero, however, is more subtle than the behavior of SISO zeros. G(s) need not have 
transmission-zeros at the zeros of the elements of $\mathrm{G}(\mathrm{s})$; and, conversely, a transmission-zero of $\mathrm{G}(\mathrm{s})$ may fail to be a zero of any individual element. Algebraically, $\mathrm{G}(\mathrm{s})$ will have a transmission zero at $s_{1}$ iff every minor of some given order has a zero at $s_{1}$. The value $s_{1}$ which appears as a transmission-zero of $\mathrm{G}(\mathrm{s})$ may as well appear among $\mathrm{G}(\mathrm{s})$ 's poles -- without cancellation! Such is the complexity of the notion of pole-zero cancellation in the multivarible case, that a MIMO plant may contain coincident pole-zero pairs -- some of which do and some of which do not cancel as the overall transfer function is formed.

Any root of the Greatest Common Divisor of the numerators of all the entries of $\mathrm{G}(\mathrm{s})$, is called a blocking zero of $G(s)$. Clearly, $s_{0}$ is blocking-zero of $G(s)$ iff $G\left(s_{0}\right)$ is a null matrix. The blocking zeros appear as well as zeros of the smallest invariant factor of the matrix numerator $\mathrm{N}(\mathrm{s})$ in any coprime factorization of $\mathrm{G}(\mathrm{s})$. Thus, every blocking-zero is also a transmission-zero; however, except for the special case of SISO systems, the converse does not in general hold. The blocking-zeros behave like the zeros of the scalar transfer function, in that, assuming each element of $G(s)$ to be a reduced-form fraction over $R[s]$, a blocking-zero and a pole can never be coincident. In the sequel, "zero" shall refer to "transmission-zero" rather than "blocking-zero", unless expressly otherwise stated.

The Characteristic Polynomial (abbrev: CP) of G(s) can be defined as either (A) $\operatorname{det}(D(s))$, where $D(s)$ is any right- or left- matrix-denominator in a coprime factorization of $G(s)$, or (B) the product of the $a_{j}(s)$ denominator-polynomials in the McMillan Form of $G(s)$. The roots of the Characteristic Polynomial are thus the poles of $G(s)$. Operationally, the Characteristic Polynomial of $\mathrm{G}(\mathrm{s})$ is the Least Common Multiple of the denominators of all nonzero minors of $\mathrm{G}(\mathrm{s})$ of all orders, assuming each of these minors to have been expressed at the outset as a reduced-form fraction over $R[s]$. The McMillan Degree of $G(s)$ is defined as the degree of the Characteristic Polynomial of $\mathrm{G}(\mathrm{s})$. 
2.2 POLYNOMIAL MATRIX DESCRIPTION OF THE LINEAR DYNAMICAL SYSTEM

Rosenbrock's Polynomial-Matrix Description (PMD) of a multivariable linear dynamical system has the canonical form:

$$
\begin{gathered}
T(p) z(p)=U(p) u(p) \\
y(p)=V(p) z(p)+W(p) u(p)
\end{gathered}
$$

In (4) $\mathrm{T}, \mathrm{U}, \mathrm{V}$, and $\mathrm{W}$ are polynomial matrices in the differential operator and $\mathrm{T}$ is nonsingular in $M(R(p))$. The dynamical order of system (4) is defined as the degree of $\operatorname{det}(T(p))$ and will henceforth be represented by $\mathbf{n}$. $\mathbf{r}$ will represent the dimension of $\mathbf{T}$. It is assumed, for simplicity, that $r \geq n$.

\subsubsection{Redaction of the PMD into Canonical System-Matrix Form}

From system (4), a canonical "System Matrix" is assembled, as follows:

$$
P(s)=\left[\begin{array}{cc}
T(s) & U(s) \\
-V(s) & W(s)
\end{array}\right]
$$

The system Matrix can be viewed as having been generated by the following system of linear equations in the s-domain:

$$
\mathrm{P}(\mathrm{s})\left[\begin{array}{c}
\mathrm{z}(\mathrm{s}) \\
-\mathrm{u}(\mathrm{s})
\end{array}\right]=\left[\begin{array}{c}
0 \\
-\mathrm{y}(\mathrm{s})
\end{array}\right]
$$

The matrix function:

$$
\mathrm{G}(\mathrm{s})=\mathrm{V}(\mathrm{s}) \mathrm{T}(\mathrm{s})^{-1} \mathrm{U}(\mathrm{s})+\mathrm{W}(\mathrm{s})
$$


is defined to be the Transfer-Matrix associated with (5). (7) in fact describes the s-domain transfer function between $u$ and $y$ in system (4).

The PMD and its associated System Matrix serve as a common framework in which various descriptions of a linear dynamical system are presentable. For the system presented in StateSpace description, (5) takes the particular form:

$$
P(s)=\left[\begin{array}{cc}
s I-A & B \\
-C & D
\end{array}\right]
$$

The Transfer-Matrix associated with (8) is, of course:

$$
G(s)=C(s I-A)^{-1} B+D
$$

For the system given in the form of Matrix-Fraction Description (MFD):

$$
P(s)=\left[\begin{array}{cc}
D(s) & I \\
-N(s) & 0
\end{array}\right] \quad \text { or } \quad P(s)=\left[\begin{array}{cc}
\bar{D}(s) & \bar{N}(s) \\
-I & 0
\end{array}\right]
$$

and the corresponding Transfer Matrix is:

$$
\mathrm{G}(\mathrm{s})=\mathrm{N}(\mathrm{s}) \mathrm{D}(\mathrm{s})^{-1} \quad \text { or } \quad \mathrm{G}(\mathrm{s})=\overline{\mathrm{D}}(\mathrm{s})^{-1} \overline{\mathrm{N}}(\mathrm{s})
$$

By definition, the PMD (4) is irreducible and the associated System Matrix (5) has least order if:

$$
(\mathrm{T}(\mathrm{s}), \mathrm{U}(\mathrm{s})) \text { is left-coprime and } \quad(\mathrm{T}(\mathrm{s}), \mathrm{V}(\mathrm{s})) \text { is right-coprime }
$$

A number of equivalent algebraic criteria for condition (12) exist, including: $\left(i_{a}\right)$ [T $U$ ] has the Smith form [I 0], (ii $i_{2}$ [ $T \mathrm{U}$ ] has rank $\mathrm{r}$ for all complex values of $\mathrm{s}$, and ( $\mathrm{iii}_{\mathrm{a}}$ ) there exist rightcoprime polynomial matrices $(X(s), Y(s))$ to satisfy the Bezout Identity, $T X+U Y=I$. 
Analogous criteria $\left(\mathrm{l}_{\mathrm{b}}\right),\left(\mathrm{ii}_{\mathrm{b}}\right)$ and $\left(\mathrm{iii}_{\mathrm{b}}\right)$, pertaining to right-coprimeness and the $(\mathrm{T}, \mathrm{V})$ pair, can likewise be formulated. For the special case of System Matrices in State Space form, the condition corresponding to (12) reads:

$$
((\mathrm{sI}-\mathrm{A}), \mathrm{B}) \text { left-coprime } \quad \text { and } \quad((\mathrm{SI}-\mathrm{A}), \mathrm{C}) \text { right-coprime }
$$

For the System Matrix (8) to have least order, its underlying realization must thus be both controllable and observable -- in State-Space language, the realization must be "minimal".

\subsubsection{Decoupling Zeros and Reduction of the System Matrix to Least Order}

Suppose now that a given system-realization is not irreducible. Should this be a StateSpace realization, it would then have modes which cannot be actuated from the input and/or modes which cannot be detected at the output -- including, possibly, modes which are jointly uncontrollable and unobservable. For the general polynomial-matrix-described system, Rosenbrock speaks, correspondingly, of "input-decoupling zeros" (i.d. zeros), "output-decoupling zeros" (o.d. zeros) and "input-output decoupling zeros" (i.o.d. zeros). Algebraically, the i.d. zeros are defined as the zeros of invariant factors in the Smith Form of [T U]. The o.d. zeros are defined as zeros of invariant factors in the Smith Form of $\left[T^{t} V^{t}\right]^{t}$ It follows from the definition (12) that the general System Matrix of type (5) has least order if and only if it has no decoupling zeros.

Any non-irreducible PMD can be reduced to least order by having its decoupling zeros "removed". Let $s_{0}$ be an i.d. zero of $(5)$, so that $\left[T\left(s_{0}\right) U\left(s_{0}\right)\right]$ has rank strictly less than $r$. We suppose, for simplicity, that $s_{0}$ is real. There then exists a constant matrix $K$ such that in $K[T(s) U(s)]$ one of the rows (say, row $\mathrm{j}$ ) is divisible by $\left(\mathrm{s}-\mathrm{s}_{0}\right)$. Substituting $[\mathrm{KT} \mathrm{KU}]$ for $[\mathrm{T} U]$ in the System 
Matrix $P(s)$ and then dividing row $j$ by $\left(s-s_{0}\right)$ yields a new System Matrix $P_{1}(s)$, associated with the same $G(s)$, but having dynamic order exactly one lower. An analogous dual procedure for removing o.d. zeros can likewise be described.

Next, the i.o.d. zeros can receive their algebraic characterization. We may suppose that by the above procedure all i.d. zeros have been removed from $\mathrm{P}$, yielding the System Matrix $\mathrm{P}_{2}$. Suppose further that the set of o.d. zeros of $P_{2}$ is $\left\{\theta_{1, \ldots . .,}, \theta_{b}\right\}$ and that the set of o.d. zeros of $P$ is \{ $\left.\gamma_{1}, \ldots, \gamma_{c}\right\}$. Clearly, $\left\{\left(\theta_{i}\right)\right\}$ is a subset of $\left\{\left(\gamma_{i}\right)\right\}$. The i.o.d. zeros of $P$ are now defined as the setdifference $\left\{\left(\gamma_{i}\right)\right\}-\left\{\left(\theta_{i}\right)\right\}$. Operationally, they are those o.d. zeros of $P$ which no longer show up as o.d. zeros after the i.d. zeros have been removed. Conceptually, they are those zeros which are associated with the output as well as with the input. The i.o.d. zeros will be represented henceforth by the set $\left\{\delta_{1}, \ldots . ., \delta_{d}\right\}$.

When a State-Space realization fails to be minimal, it is well known that the modes of the system strictly outnumber the poles of the transfer function. For the general PMD, Rosenbrock identifies the modes of the system with the set of zeros of $\operatorname{det}(\mathrm{T}(\mathrm{s}))$, a set which he enumerates by $\left(\zeta_{1}, \ldots, \zeta_{n}\right)$. Correspondent to this specification, the set of poles of the associated transfer matrix, $\mathrm{G}(\mathrm{s})$, is given by:

$$
\left\{\left(\alpha_{i}\right)\right\}=\left\{\left(\zeta_{\mathrm{i}}\right)\right]-\left(\left[\left(\beta_{\mathrm{i}}\right),\left(\gamma_{\mathrm{i}}\right)\right]-\left\{\left(\delta_{\mathrm{i}}\right)\right\}\right)
$$

We may suppose, for specificity, that the order of the set $\left\{\left(\alpha_{i}\right)\right\}$ is a. Operationally, the poles of the transfer function are those modes of the system which remain after the system has been reduced as far as possible. Algebraically, what reduces the number of poles of $G(s)$ from the number of zeros of $\operatorname{det}(\mathrm{T}(\mathrm{s}))$ is, specifically, the total number of decoupling zeros. Since every i.o.d. zero is both an i.d. zero and an o.d. zero, the right-hand-most expression in (14) prevents the possible double book-keeping of having one and the same i.o.d. zero counted twice. A formula corresponding to definition (14) is: 


$$
a=n-((b+c)-d)
$$

The number of poles of the transfer matrix equals the number of modes of the system minus the number of decoupling zeros.

\subsubsection{Strict System Equivalence of System Matrices}

Rosenbrock sought the convenience of representing transformations of the system equations by corresponding operations on the System Matrix. Naturally, those transformations in which basic system-theoretic properties are preserved hold the highest interest in this regard.

System Matrices $P_{1}$ and $P_{2}$ are defined by Rosenbrock to be Strictly System Equivalent (SSE) if they can be related by a transformation of the sort:

$$
\left[\begin{array}{cc}
M(s) & 0 \\
X(s) & I
\end{array}\right]\left[\begin{array}{cc}
T_{1}(s) & U_{1}(s) \\
-V_{1}(s) & W_{1}(s)
\end{array}\right]\left[\begin{array}{cc}
N(s) & Y(s) \\
0 & I
\end{array}\right]=\left[\begin{array}{cc}
T_{2}(s) & U_{2}(s) \\
-V_{2}(s) & W_{2}(s)
\end{array}\right]
$$

In (16) $\mathrm{M}, \mathrm{N}, \mathrm{X}$ and $\mathrm{Y}$ are polynomial matrices, with $\mathrm{M}$ and $\mathrm{N}$ by requirement unimodular. Between systems in State-Space form, Strict System Equivalence corresponds to the familiar relation of System Similarity, in which:

$$
\left[\begin{array}{cc}
\mathrm{H} & 0 \\
0 & \mathrm{I}
\end{array}\right]\left[\begin{array}{cc}
\mathrm{sI}-\mathrm{A}_{1} & \mathrm{~B}_{1} \\
-\mathrm{C}_{1} & \mathrm{D}_{1}
\end{array}\right]\left[\begin{array}{cc}
\mathrm{H}^{-1} & 0 \\
0 & \mathrm{I}
\end{array}\right]=\left[\begin{array}{cc}
\mathrm{sI}-\mathrm{A}_{2} & \mathrm{~B}_{2} \\
-\mathrm{C}_{2} & \mathrm{D}_{2}
\end{array}\right]
$$

In (17), $H$ is required to be a nonsingular matrix over the Complex Field.

Rosenbrock shows that any relation of Strict System Equivalence can be generated by a finite sequence of elementary operations of the following types: (i) multiplication of any of the first $\mathrm{r}$ rows (columns) by a unit of $\mathrm{R}[\mathrm{s}$ ], i.e. by a nonzero constant, (ii) addition of a multiple, by 
an arbitrary element of $R[s]$, i.e. by an arbitrary polynomial, of any of the first $r$ rows (columns) to any other row (column), and (iii) interchange of any two among the first $r$ rows (columns). If $P_{1}$ and $P_{2}$ are SSE, then clearly they will have the same dimension and demonstrably they will have the same Transfer Matrix. Rosenbrock's detailed study of deep algebraic structure reveals that, in fact, the Smith forms of each of $T(s),[T(s) U(s)]$ and $\left[T(s)^{2} V(s)^{t}\right]^{2}$ are preserved under Strict System Equivalence. Consequently, the sets of i.d., o.d. and i.o.d. zeros and the set of poles of the system (likewise, the set of poles of the transfer matrix) are all SSE-invariant properties.

From these results, a conclusion is drawn, whose importance merits statement in Theorem form:

Theorem 1: If $P_{1}$ and $P_{2}$ are irreducible System Matrices, then $P_{1}$ and $P_{2}$ are SSE if and only if they are associated with the same $G(s)$.

In Theorem $1, P_{1}$ and $P_{2}$ are not required to be same-form system descriptions. It follows from the Theorem that under the irreducibility assumption, each of State-Variable, Matrix Fraction and Polynomial Matrix descriptions has the capability of representing $G(s)$ with the same essential information. If three different irreducible realizations of the same $G(s)$ were at hand, the first in the form of State-Space, the second of MFD and the third of general PMD description, the following important relationships would then hold among the three:

$$
\begin{gathered}
\operatorname{det}((\mathrm{SI}-\mathrm{A}) \sim \operatorname{det}(\mathrm{D}(\mathrm{s})) \sim \operatorname{det}(\mathrm{T}(\mathrm{s})) \sim \mathrm{CP}(\mathrm{G}(\mathrm{s})) \\
\operatorname{dim}(\mathrm{A})=\operatorname{deg}(\operatorname{det}(\mathrm{D}(\mathrm{s})))=\mathrm{r}=\mathrm{McMillan} \text { degree of } \mathrm{G}(\mathrm{s}) .
\end{gathered}
$$

In (18a), " " denotes the relation of equality up to multiplication by a unit of $R$ [s], i.e. up to multiplication by a constant. 


\subsubsection{System Matrices for Closed-Loop Configurations}

System Matrices for general systems in closed-loop feedback configurations can also be constructed. We here consider the simple feedback configuration of Figure 6 , a cascade of two multivariable systems enclosed in a unity feedback loop:

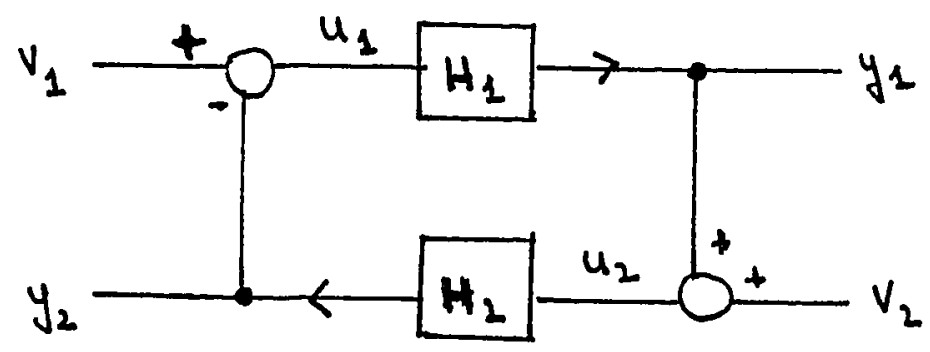

Figure 6

Rosenbrock showed that a system Matrix for this configuration can be written, in terms of the component System Matrices $P_{i}=\left[\begin{array}{cc}T_{i} & U_{i} \\ -V_{i} & W_{i}\end{array}\right](i=1,2)$ corresponding to $H_{1}$ and $H_{2}$, as:

$$
\mathrm{P}_{\mathrm{cl}}=\left[\begin{array}{ccccccccc}
\mathrm{T}_{1} & \mathrm{U}_{1} & 0 & 0 & 0 & 0 & 0 & 0 \\
0 & 0 & \mathrm{~T}_{2} & \mathrm{U}_{2} & 0 & 0 & 1 & 0 & 0 \\
-\mathrm{V}_{1} & \mathrm{~W}_{1} & 0 & 0 & -\mathrm{I} & 0 & 1 & 0 & 0 \\
0 & 0 & -\mathrm{V}_{2} & \mathrm{~W}_{2} & 0 & -\mathrm{I} & 0 & 0 \\
0 & \mathrm{I} & 0 & 0 & 0 & \mathrm{I} & -\mathrm{I} & 0 \\
0 & 0 & 0 & \mathrm{I} & -\mathrm{I} & 0 & 1 & 0 & -\mathrm{I} \\
\hdashline-\mathrm{V}_{1} & \mathrm{~W}_{\mathrm{I}} & 0 & 0 & 0 & 0 & 0 & 0 \\
0 & 0 & -\mathrm{V}_{2} & \mathrm{~W}_{2} & 0 & 0 & 1 & 0 & 0
\end{array}\right] \equiv\left[\begin{array}{cc}
\mathrm{T}_{\mathrm{CL}} & \mathrm{U}_{\mathrm{CL}} \\
-\mathrm{V}_{\mathrm{CL}} & \mathrm{W}_{\mathrm{CL}}
\end{array}\right]
$$

With $\left[v_{1}^{t} v_{2}^{t}\right]^{t}$ considered as the overall input vector, $\left[y_{1}^{t} y_{2}^{t}\right]^{t}$ the overall output vector and $\left[x_{1}^{t} x_{2}^{t} u_{1}^{t} u_{2}^{t} y_{1}^{t} y_{2}^{t}\right]^{t}$ as vector of the internal state (or "pseudo-state"), all the relevant PMD-form system equations are readily verified to be contained in the matrix equation: 


$$
P_{C Z}\left[x_{1}^{t}-u_{1}^{t} x_{2}^{t}-u_{2}^{t}-y_{1}^{t}-y_{2}^{t} \quad l \quad-v_{1}^{t}-v_{2}^{t}\right]^{t}=\left[\begin{array}{lll}
0^{t} & 1 & -y_{1}^{t}-y_{2}^{t}
\end{array}\right]^{t} \text {. }
$$

$\mathrm{H}_{1}(\mathrm{~s})$ and $\mathrm{H}_{2}(\mathrm{~s})$ are here assumed to be proper transfer matrices. $\mathrm{P}_{1}(\mathrm{~s})$ and $\mathrm{P}_{2}(\mathrm{~s})$, by assumption, provide least-order representations for them. "Well-posedness" of the closed-loop system, the guarantee that a given input shall determine a unique output, then requires that $(I+$ $\mathrm{H}_{1}(\mathrm{~s}) \mathrm{H}_{2}(\mathrm{~s})$ ) be nonsingular over $\mathrm{R}(\mathrm{s})$ (i.e. that $\operatorname{det}\left(\mathrm{I}+\mathrm{H}_{1}(\mathrm{~s}) \mathrm{H}_{2}(\mathrm{~s})\right) \neq 0$ ); while "regularity", the guarantee that the closed-loop transfer matrix shall remain proper as well, requires, more restrictively, that $\left(\mathrm{I}+\mathrm{H}_{1}(\infty) \mathrm{H}_{2}(\infty)\right)$ be nonsingular over the Complex Field (i.e. that det $\left(\mathrm{I}+\mathrm{H}_{1}(\infty) \mathrm{H}_{2}(\infty)\right.$ ) $\neq 0$ )). Given well-posedness and regularity, the following important relationship between poles of open- and closed-loop systems was shown by Rosenbrock to hold:

$$
\operatorname{det}\left(\mathrm{T}_{\mathrm{cl}}(\mathrm{s})\right)=\operatorname{det}\left(\mathrm{T}_{1}(\mathrm{~s})\right) \operatorname{det}\left(\mathrm{T}_{2}(\mathrm{~s})\right) \operatorname{edet}\left(\mathrm{I}+\mathrm{H}_{1}(\mathrm{~s}) \mathrm{H}_{2}(\mathrm{~s})\right)
$$

\subsection{W. LUSE'S ANALYSIS OF THE MULTIPLE FREQUENCY SCALE SYSTEM}

In the work of D.W. Luse [18-21], the matrix function, $\mathrm{H}(\mathrm{s}, \mathrm{e})$, of the complex frequency variable $s$ and the parameter $e$ is studied. Since $H(s, e)$ is to be the transfer matrix of a finitedimensional linear time-invariant dynamical system whose behavior depends upon a small parameter, it is to be rational in $\mathrm{s}$, and $\mathrm{e}$ is to be restricted to the range $\left[0, \mathrm{e}^{*}\right)$ for some small upper bound $\mathrm{e}^{*}$. Elsewise, a very general e-dependence is permitted. The sole restriction on $\mathrm{e}$ is that the coefficients of $s$ be taken from the Field:

$$
F_{e}=\left\{\frac{f(e)}{e^{m}}: f \text { is analytic at } e=0, m \text { is a pos. integer }\right\}
$$

$F_{e}$ is, namely, the Field of functions of e whose Laurent Expansion about $e=0$ has only a finite 
number of negative powers of $e$.

The coefficients are not required to be strictly analytic at $e=0$. However, in the case that the coefficients of an entry of $H(s, e)$ in fact have poles at $e=0$, the simple multiplication of the numerator and denominator of this entry by the suitable power of e suffices to remove all the negative powers from them. Thus, without loss of generality, we henceforth assume any matrix given as rational in $s$ over $F_{e}$ to have been normalized for e-analytic coefficients and to be nontrivially evaluable when e is set to zero. For any value $e_{1}$ of $e, H\left(s, e_{1}\right)$ will now be a rational matrix in s over the Complex Field.

\subsubsection{Algebraic Criteria for the Multiple-Frequency-Scale Property}

Luse submits a set of necessary and sufficient criteria for identifying the $H(s, e)$ of the foregoing type which displays two-frequency-scale behavior. At the outset, it is hypothesized that $H(s, e)$ is proper in s. The First Criterion then requires: that both $H(s, 0)$ and $H((p / e), e) ~ I ~ b e$ defined and proper over the Complex Field. $H((p / e), e)$ is simply a version of $H(s, e)$ scaled to the "fast" frequency variable:

$$
p=e s
$$

The $0(1)$ values of $p$ denote the same frequency range as the $0(1 / \mathrm{e})$ values of $s$. That is to say, the same frequency which looks like a very high frequency to $s$ looks like a low or normal frequency to $\mathrm{p}$. Thus, $\mathrm{p}$ is regarded as a "fast" frequency variable.

A Second Criterion is presented by Luse in several equivalent versions. A first version of the Second criterion is phrased in terms of the poles of $\mathrm{H}(\mathrm{s}, \mathrm{e})$. A second version, which does not require these poles to be computed, is phrased in terms of the Characteristic Polynomial of $\mathrm{H}(\mathrm{s}, \mathrm{e})$. 
A third version of the Second Criterion, not requiring even the Characteristic Polynomial to be computed, is phrased in terms of an algebraic form which each entry of $H(s, e)$ must satisfy. In this version, the Second Criterion requires that each $h_{i j}(s, e)$ be expressable as:

$$
h_{i j}(s, e)=\frac{n_{1 i j}(s, e)+s^{k} n_{2 i j}(e s, e)}{d_{1 i j}(s, e)+s^{k} d_{2 i j}(e s, e)}
$$

In (24), it is required that: (i) all four polynomials have coefficients analytic at $e=0$; (ii) $k=$ deg $\left(d_{1}(s, e)\right)=\operatorname{deg}\left(d_{1}(s, 0)\right) \geq \operatorname{deg}\left(n_{1}(s, e)\right) ;\left(\right.$ iii) $\operatorname{deg}\left(d_{2}(p, e)\right)=\operatorname{deg} d_{2}(p, 0) \geq \operatorname{deg} n_{2}(p, e) ;$ and (iv) the constant terms of $n_{2}$ and $d_{2}$ are both zero. (It is here assumed that before expansion into the form of $(24), h_{i j}(s, e)$ represents a coprime fraction over the Ring of polynomials with coefficients in $F_{e \cdot}$ )

In effect, Luse's Criteria provide an algebraic characterization (different from the characterization based directly on spectral grounds) of the situation in which $\mathrm{H}(\mathrm{s}, \mathrm{e})$ 's e-dependent poles can be clustered into distinct groups having disparate orders of magnitude. Such an $H(s, e)$ then behaves, in each of two distinct frequency ranges, like a distinct parameter-independent lowerorder dynamical system. Luse defines the $H_{S}(s)\left(S\right.$ for "slow") and the $H_{F}(p)(F$ for "fast"), which approximately describe $\mathrm{H}(\mathrm{s}, \mathrm{e})$ 's respective low-frequency and high-frequency behaviors, by:

$$
\mathrm{H}_{\mathrm{S}}(\mathrm{s}) \equiv \mathrm{H}(\mathrm{s}, 0) \quad \text { and } \quad \mathrm{H}_{\mathrm{F}}(\mathrm{p}) \equiv \mathrm{H}((\mathrm{p} / \mathrm{e}), \mathrm{e}) \underset{\in \rightarrow 0}{\mathrm{l}}
$$

Note that these two system descriptions are written in different frequency scales. When $\mathrm{H}(\mathrm{s}, \mathrm{e})$ satisfies Luse's Criteria, $H_{S}(s)$ and $H_{F}(p)$ are in fact well-defined and proper, and they may have no poles in common. By (24), it is further guaranteed that:

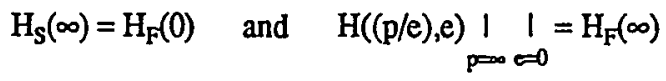


(26) provides matching-conditions for the point at which the adjacent frequency-ranges meet and for the point at which the higher frequency-scale meets the given $\mathrm{H}(\mathrm{s}, \mathrm{e})$ at infinity. These matching conditions are strictly valid when the parameter is zero and approximately valid when the parameter is small. Since $\mathrm{H}_{\mathrm{S}}(\mathrm{s})$ is proper, the matching conditions specifically rule out the possibility of $\mathrm{H}_{\mathrm{F}}(\mathrm{p})$ 's having poles at the origin.

Luse [21] has also generalized the above results to accomodate the multiple (i.e. three or more) frequency scale systems. His First Criterion in such cases generalizes to:

$$
H^{j}(p)=H\left(\left.\left(p / e^{j-1}, e\right)\right|_{e=0} \text { is defined and proper } \quad(j=1, \ldots, n+1)\right.
$$

Each of these $n+1$ e-independent lower-order systems now provides an approximate description of $\mathrm{H}(\mathrm{s}, \mathrm{e})$, the $\mathrm{j}^{\text {th }}$ system description having validity as an approximation in the $\mathrm{j}^{\text {th }}$ frequency range. Again, the descriptions for different frequency ranges can have no poles overlapping, and matching-conditions for adjacent frequency ranges (including the matching of $\mathrm{H}(\mathrm{s}, \mathrm{e})$ at the highest frequency with its highest frequency scale) are again guaranteed. For this multiplefrequency-scale system, the matching conditions take the form:

$$
H^{j}(\infty)=H^{j+1}(0) \quad(j=1, \ldots, n) \quad \text { and } \quad H((p / e), e) \text { । } \quad \mid=H^{n+1}(\infty)
$$

Specifically, $\mathrm{H}^{\mathrm{j}}(\mathrm{p})$ may have no poles at the origin for $\mathrm{j}=2, \ldots, \mathrm{n}+1$.

Luse further defines:

$$
\begin{aligned}
& \text { Lost Slow Poles of } \mathrm{H}(\mathrm{s}, \mathrm{e})=\text { roots of } \frac{\mathrm{CP}(\mathrm{H}(\mathrm{s}, \mathrm{e})) \underset{\mathrm{e}}{\mathrm{l}}}{\mathrm{CP}\left(\mathrm{H}_{\mathrm{S}}(\mathrm{s})\right)} \\
& \text { Lost Fast Poles of } H(s, e)=\text { nonzero roots of } \frac{\mathrm{CP}(\mathrm{H}((\mathrm{p} / \mathrm{e}), \mathrm{e})) \underset{\mathrm{\omega}}{\mathrm{l}}}{\mathrm{CP}\left(\mathrm{H}_{\mathrm{F}}(\mathrm{p})\right)}
\end{aligned}
$$


Note that in forming the numerators of these two fractons, first a Characteristic Polynomial is determined and then the parameter is set to zero; in forming the denominators, first the parameter is set to zero and then a Characteristic Polynomial is formed. Between these two procedures, an order of operations is reversed.

\subsubsection{Analogy between Frequency-Domain and Time-Domain Results}

It is apparent that Luse provides a frequency-domain analogue to the multiple time-scales and associated trajectory-approximating reduced-order models of Coderch, Willsky, Sastry amd Castanon [3], which were reviewed in Chapter I. Luse's Criteria for identifying and then decomposing a system's multiple-frequency-scale behavior, which are based on the algebraic properties of e-dependent transfer matrices, substitute in the frequency domain for the complicated perturbed-operator-theoretic iterative algorithm. The fact that each of the polynomials whose roots are defined as "lost-poles" may be nontrivial, i.e. the fact that some of the "normal" poles of the multiple-frequency-scale system may get "lost" at $e=0$, corresponds to the time-domain reduction of order of the singularly perturbed system at this distinguished value of the parameter.

Luse provides a structure for the approximation of the given $\mathrm{H}(\mathrm{s}, \mathrm{e})$ by the lower-order $\mathrm{H}_{S}$ (s) and $\mathrm{H}_{\mathrm{F}}$ (es), just as Coderch et al have provided bounds for the trajectory-approximating properties of the time-domain reduced-order models. Luse's determination on this matter has sufficient importance to merit statement in Theorem form:

Theorem 2: Provided that $\mathrm{H}_{S}(\mathrm{~s})$ and $\mathrm{H}_{\mathrm{F}}(\mathrm{p})$ have no purely imaginary poles and $\mathrm{H}(\mathrm{s}, \mathrm{e})$ has no purely imaginary lost poles:

$$
\sup _{w \in R}\left\|H(j w, e)-\left(H_{S}(j w)+H_{F}(j e w)-H_{S}(\infty)\right)\right\|=O(e)
$$


Theorem 2 will be referred to henceforth as "Luse's Approximation Result". In (31), "II II" stands for any matix norm on $\mathrm{C}^{\mathrm{p} \times \mathrm{q}}$. By Theorem $2, \mathrm{H}_{S}(\mathrm{~s})$ and $\mathrm{H}_{\mathrm{F}}(\mathrm{p})$ together provide a uniform (i.e.

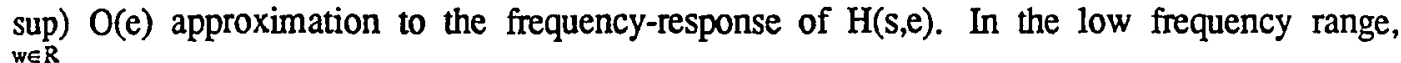
$H_{F}(j e w)$ looks like $H_{F}(0)$. By the matching condition, $H_{S}(\infty)=H_{F}(0)$; consequently, $\| \mathrm{H}(j w, e)-H_{S}$ (jw) $\|$ is small, i.e. $H(j w, e)$ looks like $H_{S}(j w)$. In the high frequency range, $H_{S}(j w)$ looks like $H_{S}(\infty)$ : consequently, $\left\|\mathrm{H}(j w, e)-H_{F}(j e w)\right\|$ is small, i.e. $H(j w, e)$ looks like $H_{F}(j e w)$. When $H_{S}(s)$ and $H_{F}(p)$ have no poles in fact over the entire closed right-half-plane, and also $H(s, e)$ has no closed-right-half-plane lost poles, the Maximum Modulus Theorem guarantees Luse's Approximation Result to hold over that entire region, i.e.:

$$
\sup _{\operatorname{Re} s \geq 0}|| H(s, e)-\left(H_{S}(s)+H_{F}(e s)-H_{S}(\infty)\right) \| \mid=0(e)
$$

In principle, an exact decomposition for the multiple-frequency-scale $\mathrm{H}(\mathrm{s}, \mathrm{e})$ is also possible. The "Laplace Expansion" for a proper rational function, $h(s)$, with coefficients over an arbitrary Field is given by:

$$
h(s)=\frac{n(s)}{f_{0}(s) f_{1}(s) . f_{n}(s)}=\frac{r_{0}(s)}{f_{0}(s)}+\ldots . .+\frac{r_{n}(s)}{f_{n}(s)}+k
$$

In (33) $n, f_{0}, \ldots, f_{s}$ are mutually coprime polynomials with coefficients over the given Field, $\mathrm{k}$ is a (uniquely determined) Field element and each of the (uniquely determined) rational functions $r_{j} / f_{j}$ is strictly proper. Since the Expansion applies to rational functions with coefficients over an arbitrary Field, it is guaranteed to apply to rational functions with coefficients over $F_{e}$. In this light, the proper multiple-frequency-scale $\mathrm{H}(\mathrm{s}, \mathrm{e})$ must be exactly and uniquely decomposable as:

$$
H(s, e)=H_{1}(s, e)+H_{2}(e s, e)+\ldots . .+H_{n+1}\left(e^{n} s, e\right)+A(e)
$$


In (34), $\mathrm{A}$ is a constant matrix of e-dependent Field elements and the other matrices are all strictly proper in s, each of them having its poles in precisely one of the characterizable frequency ranges. This exact decomposition, involving the factorization of polynomials with variable coefficients, might not be computable in given cases. The Approximation Result, on the other hand, is always readily utilizable. Here is again an analogue to the time-domain results of Coderch, Willsky, Sastry and Castanon. The latter authors do not work with the exact blockdiagonalization of the state-transition matrix of the multiple-time-scale system. They work rather with parameter-independent simplified models of the "true" blocks, which are obtainable through the constructive procedure of the iterative algorithm, and which serve well enough for the purposes of trajectory-approximation when the value of the parameter is small.

\subsubsection{Representation of the Explicit Singularly Perturbed System}

In an important special case, $\mathrm{H}(\mathrm{s}, \mathrm{e})$ stands for the transfer matrix of the explicitly singularly perturbed system. That system is here re-introduced as system (35):

$$
\begin{gathered}
{\left[\begin{array}{c}
\dot{x} \\
\dot{e} z
\end{array}\right]=\left[\begin{array}{cc}
A_{11} & A_{12} \\
A_{21} & A_{22}
\end{array}\right]\left[\begin{array}{l}
x \\
z
\end{array}\right]+\left[\begin{array}{l}
B_{1} \\
B_{2}
\end{array}\right] u} \\
y=C_{1} x+C_{2} z
\end{gathered}
$$

The Rosenbrock System Matrix for system (35) is, clearly:

$$
P(s, e)=\left[\begin{array}{cc:c}
s I-A_{11} & -A_{12} & B_{1} \\
-A_{21} / e & s I-A_{22} / e & B_{2} / e \\
--- & --- & --- \\
-C_{1} & -C_{2} & 0
\end{array}\right]
$$

It is helpful here to recall that the system of equations: 


$$
P(s, e)\left[x^{2} z^{2} \quad 1-u^{t}\right]^{t}=\left[\begin{array}{lll}
0^{t} & 1 & -y^{t}
\end{array}\right]^{t}
$$

is precisely the set of equations which represent (35) in the Complex Frequency Domain.

In view of the defining relations presented in (25), the System Matrices for the slow and fast approximating systems $H_{S}(s)$ and $H_{F}(p)$ corresponding to system $(35)$ are $P(s, 0)$ and $\left.P((p / e), e)\right|_{c=0}$ respectively. A simple substitution of zero for e in (36) would leave non-analytic coefficients in the second row of the System Matrix. Therefore, to form the System Matrix $\mathrm{P}(\mathrm{s}, 0)$ for $\mathrm{H}_{\mathrm{s}}(\mathrm{s})$, the second row is multiplied by e before e is set to zero -- a permissible operation, since it preserves Strict System Equivalence. Likewise, to form the System Matrix $P((p / e), e) \underset{e=0}{\mid}$ for $\mathrm{H}_{\mathrm{F}}(\mathrm{p})$, after substituting $\mathrm{p} / \mathrm{e}$ for $\mathrm{e}$, both first and second rows are multiplied by e before setting e to zero. Resultantly:

$$
\begin{aligned}
& P(s, 0)=\left[\begin{array}{cc:c}
s I-A_{11} & -A_{12} & B_{1} \\
-A_{21} & -A_{22} & B_{2} \\
\hdashline-C_{1} & -C_{2} & 0
\end{array}\right]
\end{aligned}
$$

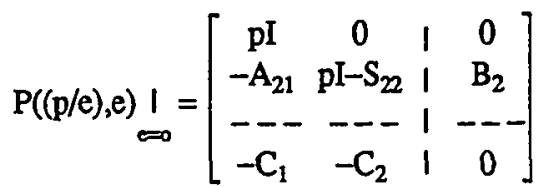

Applying (7), consistently with the rules for computing the inverse of a partitioned matrix, the transfer matrix corresponding to (38) is determined as:

$$
\begin{aligned}
H_{S}(s) & =\left(C_{1}-C_{2} A_{22}^{-1} A_{21}\right)\left(s I-\left(A_{11}-A_{12} A_{22}^{-1} A_{21}\right)\right)^{-1}\left(B_{1}-A_{12} A_{22}^{-1} B_{2}\right)-C_{2} A_{22}^{-1} B_{2} \\
& \equiv C_{0}\left(s I-A_{0}\right)^{-1} B_{0}+D_{0}
\end{aligned}
$$

and the transfer matrix corresponding to (39) is determined as: 


$$
H_{F}(p)=C_{2}\left(p I-A_{2}\right)^{-1} B_{2}
$$

Not surprisingly, $\mathrm{H}_{S}(\mathbf{s})$ is the transfer matrix of Chapter I's "reduced-order" subsystem, while $H_{F}(p)$ (with fast frequency variable $p$ corresponding to the fast time variable $\tau$ ) is the transfer matrix of Chapter I's "boundary-layer" subsystem.

\subsubsection{Representation of the General Multiple Frequency Scale System}

For adumbrating the general multiple-frequency-scale system within the System Matrix framework, Luse chooses a particular form of representation which will prove the most useful in the developments which follow. As previously shown, given the transfer matrix $H(s)$ with coefficients over Real or Complex Fields which, by hypothesis, sustains the right coprime factorization $\mathrm{H}(\mathrm{s})=\mathrm{V}(\mathrm{s}) \mathrm{T}(\mathrm{s})^{-1}$, one of the canonical forms for its System Matrix is the least-order right Matrix Fraction Description:

$$
P(s)=\left[\begin{array}{cc}
T(s) & I \\
-V(s) & 0
\end{array}\right]
$$

It is a general feature of Luse's work first to make Rosenbrock's Theory explicit for System Matrices in which the coefficients may come from an arbitrary Field, and secondly to apply the Theory to the specific case in which those coefficients lie in the Field $F_{e}$. As Luse shows, in $F_{e}$ the parameter is introduced in a way which is sufficiently flexible to enable most of the results from Rosenbrock's Theory still to be valid. Accordingly, there corresponds to any multiplefrequency-scale H(s,e) a least-order System Matrix in the right-coprime MFD form:

$$
P(s, e)=\left[\begin{array}{cc}
T(s, e) & I \\
-V(s, e) & 0
\end{array}\right]
$$


By Theorem 1, (43) must be as good as any other choice of irreducible representation, from the standpoint of conveyance of essential information about the system.

If, in the System Matrix (43) for a given system, any of the coefficients have poles at $e=0$, the appropriate columns will be multiplied by suitable powers of e to make these coefficients analytic. Let $P_{1}(\mathrm{~S}, \mathrm{e})$ then denote the System Matrix (43) with columns cleared. To the System Matrix $\mathrm{P}((\mathrm{p} / \mathrm{e}), \mathrm{e})$ which corresponds to the scaled transfer matrix $\mathrm{H}((\mathrm{p} / \mathrm{e}), \mathrm{e})$, there likewise corresponds a cleared least-order version, $\overline{\mathrm{P}}_{1}(\mathrm{p}, \mathrm{e})$. The purpose of the clearing is to ensure that $P_{1}(s, 0)$ and $\bar{P}_{1}(p, 0)$ are in fact well-defined System Matrices over the Complex Field -- representing, respectively, $H_{S}(s)$ and $H_{F}(p)$. Due to the possible occurence of "lost" poles, $P_{1}(s, 0)$ and $\overline{\mathrm{P}}_{1}(p, 0)$ may fail, however, to be least-order System Matrices. There cannot be any inputdecoupling zeros in the right-MFD construction, but output-decoupling zeros may still possibly appear. Luse thus next provides that all o.d. zeros be removed from $P_{1}(s, 0)$ to yield $P_{1 s}(s)$ and from $\bar{P}_{1}(p, 0)$ to yield $P_{1 F}(p) . P_{1 S}(s)$ and $P_{1 F}(p)$ are then in fact least-order System Matrices representing, respectively, $H_{S}(s)$ and $H_{F}(p)$ in the canonical right-MFD form.

The "lost poles", which were defined initially in (29) and (30) in terms of Characteristic Polynomials, can now be specified in terms of the specific System Matrices which have just been

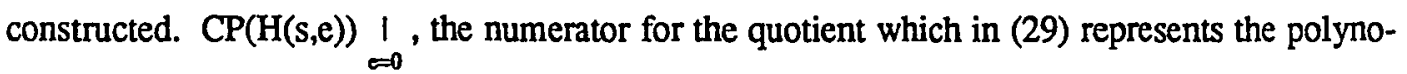
mial whose zeros are the lost slow poles, can be identified with the determinant of the upper left block, $T_{1}(s, e)$, of $P_{1}(s, e)$, evaluated at $e=0 . C P\left(H_{s}(s)\right)$, the denominator-polynomial for this quotient, can be identified with the determinant of the upper left block, $T_{1 s}(s)$, of $P_{1 s}(s)$. Due to Luse's method of construction, which has made $P_{1 S}(s)$ a least-order or "reduced" version of $P_{1}(s, 0)$, the denominator in (29) differs from the numerator only in the extraction of all decoupling zeros. Due to his choice of the right-MFD canonical form, the only possible decoupling zeros are o.d. zeros. Resultantly, through this particular choice of representations, the lost slow poles of $H(s, e)$ are displayed as the output decoupling zeros of $P_{1}(s, 0)$. In summary: 


$$
\text { lost slow poles of } H(s, e)=\text { roots of } \frac{\operatorname{det}\left(T_{1}(s, 0)\right)}{\operatorname{det}\left(T_{1 S}(s)\right)}=0 . d \text {. zeros of } P_{1}(s, 0)
$$

Working analogously with the upper left block, $\overline{\mathrm{T}}_{1}(\mathrm{p}, 0)$, of the scaled System Matrix, $\bar{P}_{1}(p, e)$, evaluated at $e=0$, and with the upper left block, $T_{1 F}(p)$, of $P_{1 F}(p)$ yields the corresponding result:

$$
\text { lost fast poles of } H(s, e)=\text { nonzero roots of } \frac{\operatorname{det}\left(\bar{T}_{1}(p, 0)\right)}{\operatorname{det}\left(T_{1 F}(p)\right)}=\text { nonzero o.d. zeros of } \bar{P}_{1}(p, 0)
$$

The lost fast poles of $\mathrm{H}(\mathrm{s}, \mathrm{e})$ are the (nonzero) output decoupling zeros of $\overline{\mathrm{P}}_{1}(\mathrm{p}, 0)$.

\subsubsection{Derivation of Luse's Closed-Loop Theorem}

We finally consider closed-loop applications in which, specifically, two-frequency-scale systems are involved.

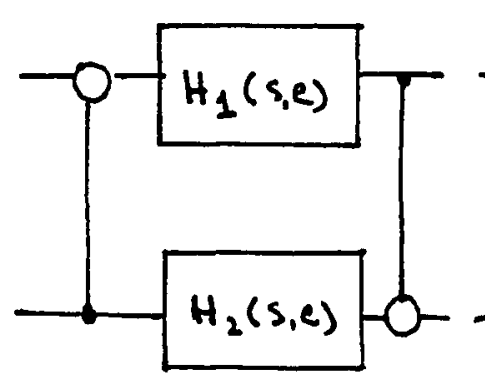

Figure 7a

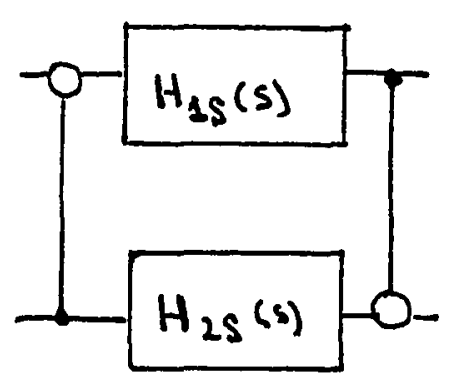

Figure $7 \mathrm{~b}$

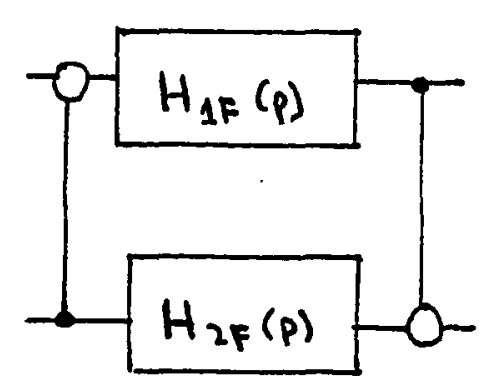

Figure 7c

In Figure $7, H_{j}(s, e)(j=1,2)$ are understood to be two-frequency-scale systems, with $H_{j s}(s)$ and $\mathrm{H}_{\mathrm{jF}}(\mathrm{p})$ their respective reduced-order low- and high-frequency approximations. The paradigmatic closed-loop System Matrix, (19), will now be filled for each of the feedback systems, 7a, 7b and 7c, as follows: 
(A) Closed-Loop system Matrix $\mathrm{P}_{\mathrm{cl}}(\mathrm{s}, \mathrm{e})$ for Figure 7a: $\mathrm{H}_{1}(\mathrm{~s}, \mathrm{e})$ is considered to be represented by the least-order right-MFD-form System Matrix $P_{1}(s, e)$ of the previous discussion, and $\mathrm{H}_{2}(\mathrm{~s}, \mathrm{e})$ likewise by $\mathrm{P}_{2}(\mathrm{~s}, \mathrm{e})$. The relevant blocks of $\mathrm{P}_{1}(\mathrm{~s}, \mathrm{e})$ and $\mathrm{P}_{2}(\mathrm{~s}, \mathrm{e})$ are then inserted into (19) to yield the closed-loop System Matrix $\mathrm{P}_{\mathrm{cl}}(\mathrm{s}, \mathrm{e})$ corresponding to the feedback system of Figure 7a.

(B) Closed-Loop System Matrix $\mathrm{P}_{\mathrm{cls}}(\mathrm{s})$ for Figure $7 \mathrm{~b}: \mathrm{H}_{1 \mathrm{~S}}(\mathrm{~s})$ is considered to be represented by the least-order right-MFD-form System Matrix $\mathrm{P}_{1 \mathrm{~s}}(\mathrm{~s})$ of the previous discussion, and $H_{2 s}(s)$ likewise by $P_{2 s}(s)$. The relevant blocks of $P_{1 s}(s)$ and $P_{2 s}(s)$ are inserted into (19) to yield System Matrix $P_{c c s}(s)$ corresponding to Figure $7 \mathrm{~b}$.

(C) Closed-Loop System Matrix $P_{\mathrm{clF}}(\mathrm{p})$ for Figure 7c: $P_{\mathrm{clF}}(\mathrm{p})$, the closed-loop System Matrix of type (19) which represents Figure 7c, is built out of the blocks of the previous discussion's least-order right-MFD-form System Matrix $P_{1 F}(p)$, representing $H_{1 F}(p)$, and the blocks of the corresponding $P_{2 F}(p)$ which represents $H_{2 F}(p)$.

(D) Closed-Loop System Matrix $\overline{\mathrm{P}}_{\mathrm{cl}}(\mathrm{p}, \mathrm{e})$ for the scaled version of Figure 7a: The closedloop System Matrix $\overline{\mathrm{P}}_{\mathrm{cl}}(\mathrm{p}, \mathrm{e})$, using for its components the blocks of the $\overline{\mathrm{P}}_{1}(\mathrm{p}, \mathrm{e})$ of the previous discussion and those of an analogous $\overrightarrow{\mathrm{P}}_{2}(\mathrm{p}, \mathrm{e})$ derived from $\mathrm{H}_{2}(\mathrm{~s}, \mathrm{e})$, represents a scaled System Matrix for the feedback system of Figure 7a.

Luse shows that the well-posedness and regularity of these four constructions are guaranteed, provided that both $\left(\mathrm{I}+\mathrm{H}_{1 \mathrm{~S}}(\infty) \mathrm{H}_{2 \mathrm{~S}}(\infty)\right)$ and $\left(\mathrm{I}+\mathrm{H}_{1 \mathrm{~F}}(\infty) \mathrm{H}_{2 \mathrm{~F}}(\infty)\right)$ are nonsingular over the Complex Field.

From the application of Rosenbrock's result (21) to the closed loop system of Figure 7b, it is determined that:

$$
\operatorname{det}\left(\mathrm{T}_{\mathrm{clS}}(\mathrm{s})\right)=\operatorname{det}\left(\mathrm{T}_{\mathrm{IS}}(\mathrm{s})\right) \operatorname{det}\left(\mathrm{T}_{2 \mathrm{~S}}(\mathrm{~s}) \propto \operatorname{det}\left(\mathrm{I}+\mathrm{H}_{1 \mathrm{~S}}(\mathrm{~s}) \mathrm{H}_{2 \mathrm{~S}}(\mathrm{~s})\right)\right.
$$


Applied to Figure $7 \mathrm{a}$ and evaluated at $\mathrm{e}=0$, the result (21) yields:

$$
\operatorname{det}\left(\mathrm{T}_{\mathrm{cl}}(\mathrm{s}, 0)\right)=\operatorname{det}\left(\mathrm{T}_{1}(\mathrm{~s}, 0)\right) \operatorname{det}\left(\mathrm{T}_{2}(\mathrm{~s}, 0)\right) \operatorname{det}\left(\mathrm{I}+\mathrm{H}_{1}(\mathrm{~s}, 0) \mathrm{H}_{2}(\mathrm{~s}, 0)\right)
$$

By the definition of $\mathrm{H}_{S}(\mathrm{~s})$, the third terms on the right hand sides of (46) and (47) are identical. By result (44) of the previous discussion, $\operatorname{det}\left(T_{j}(s, 0)\right) \quad(j=1,2)$ is the product of $\operatorname{det}\left(T_{j s}(s)\right)$ and the o.d. polynomial of $P_{j}(s, 0)$, the latter polynomial being itself the lost slow polynomial of $H_{j}(s, 0)$. Consequently:

$$
\operatorname{det}\left(\mathrm{T}_{\mathrm{cl}}(\mathrm{s}, 0)\right)=\left(\sum_{j=1}^{2}\left(\text { lost slow poly of } \mathrm{H}_{\mathrm{j}}(\mathrm{s}, 0)\right)\right) \bullet \operatorname{det}\left(\mathrm{T}_{\mathrm{clS}}(\mathrm{S})\right)
$$

With analogous reasoning, result (45) of the previous discussion is applied to the closed loop System Matrix $P_{1 F}(p)$ corresponding to Figure $7 c$ and to the scaled System Matrix $P_{c 1}(p, e)$ for the closed loop system of Figure 7a. Resultantly:

$$
\left.\operatorname{det}\left(T_{c l}(p, 0)\right)=p^{k} \bullet \stackrel{2}{(\pi=1}\left(\text { lost fast poly of } H_{j}(s, 0)\right)\right) \bullet \operatorname{det}\left(T_{c l F}(p)\right)
$$

In (49), the factor $p^{k}$ covers the requirement for $H_{F}(p)$ to have no poles at $p=0$. Specifically, $\mathrm{k}=\sum_{\mathrm{i}=1}^{2} \operatorname{deg}\left(\operatorname{det}\left(\mathrm{T}_{\mathrm{i}}(\mathrm{s}, 0)\right)\right)$.

By (48) and (49), under well-posedness and regularity conditions, the knowledge of the poles of the closed-loop slow and fast subsystems depicted in Figures $7 \mathrm{~b}$ and $7 \mathrm{c}$, together with knowledge of all the open loop lost poles, "fast" and "slow", provides exact knowledge of the poles of the overall closed-loop system pictured in Figure $7 \mathrm{a}$-- when the value of the parameter is zero. For the purposes of singular perturbational analysis in the frequency domain, paramount interest lies in the approximate knowledge of the poles of Figure 7a, for cases in which the value of the parameter is nonzero but still small. Because all the relevant quantities in the underlying 
structures vary continuously with the parameter, the attainment of such a result is possible. The Theorem which expresses this highly important and useful result will henceforth be referred to as "Luse's Closed-Loop Theorem":

Theorem 3: Assuming that $\left(\mathrm{I}+\mathrm{H}_{1 \mathrm{~s}}(\infty) \mathrm{H}_{2 \mathrm{~s}}(\infty)\right)$ and $\left(\mathrm{I}+\mathrm{H}_{1 \mathrm{~F}}(\infty) \mathrm{H}_{2 \mathrm{~F}}(\infty)\right)$ are nonsingular, all poles of the closed-loop system of Figure $7 \mathrm{a}$ are either within $(1+0(e))$ times $\left[\left(s_{j}\right)\right\}$ or within $(1+0(e))$ times $\left\{\left(p_{i} / e\right)\right\}$, where:

$\left\{\left(s_{\mathrm{i}}\right)\right\}$ denotes the set of poles of the corresponding closed-loop slow subsystem, Figure $7 \mathrm{~b}$, plus the lost slow poles of $H_{j}(s, e)(j=1,2)$, and:

$\left[\left(\mathrm{p}_{\mathrm{i}}\right)\right]$ denotes the set of poles of the corresponding closed-loop fast subsystem, Figure 7c, plus the lost fast poles of $H_{j}(s, e)(j=1,2)$.

This Theorem will be revisited and reinterpreted in Chapter IV, with a view towards that separate-and-composite dynamic output controller design strategy for the multiple-time-scale systems which is sought. 


\section{CHAPTER III}

\section{VIDYASAGAR'S FRACTIONAL REPRESENTATION OF LINEAR SYSTEMS}

This chapter looks at the work of M. Vidyasagar and his associates [29-34], most particularly at Vidyasagar's recent Control System Synthesis [32]. Before the relevant applications of this work to the problem at hand can be made, certain topological as well as algebraic preliminaries need to be covered. The algebraic matters for review extend and generalize the previous chapter's framework for frequency-domain analysis of multivariable dynamical systems. The topological matters, on the other hand, are new to the present discussion. Vidyasagar examines the singularly perturbed system in the context of topological considerations; his own interest in this system reflects, basically, a topological rather than an algebraic way of regarding it.

\subsection{THE ALGEBRAIC FORMALISM}

\subsubsection{The Ring of Stable Transfer Functions and the Ring of Stable Transfer Matrices}

Algebraically, all Vidyasagar's results rest upon the fact that the set of rational functions which have poles neither in the closed right-half-plane nor at the point at infinity -- i.e. the set of transfer functions of proper, stable, lumped, linear, time-invariant SISO systems -- forms a Commutative Domain with Identity, under the usual operations of addition and multiplication of rational functions. This basic set shall be referred to as $\mathbf{S}(\mathbf{s})$, or more abbreviatedly simply as $\mathbf{S}$, 
and $\mathrm{C}_{\mathrm{e}}^{+}$will represent the closed right-hand-plane including the point at infinity. With the function:

$$
\operatorname{deg}(\mathrm{f})=\# \text { of } \mathrm{C}_{e}^{+} \text {zeros of } \mathrm{f}(\mathrm{s})
$$

defined on $\mathrm{S}$ and taken for degree function, $\mathrm{S}(\mathrm{s})$ becomes an Euclidean Domain. Because it is an Euclidean Domain, it is ipso facto a Principal Ideal Domain and an Unique Factorization Domain.

The units of $S$, as elements of degree zero, are those members which have no $\mathrm{C}_{e}^{+}$zeros, i.e. those SISO transfer functions which are minimum phase and properly invertible. Clearly, $f$ is a divisor of $\mathrm{g}$ in $\mathrm{S}$ iff for every $\mathrm{C}_{\mathrm{e}}^{+}$zero of $\mathrm{f}, \mathrm{g}$ has the same zero with at least the same multiplicity. Furthermore, $(f, g)$ will be coprime in $S$ iff they have no common $\mathrm{C}_{e}^{+}$zeros, i.e. if they have no common right-half-plane zeros and at least one of them has relative degree zero. Since $S$ is a Principal Ideal Domain, any two elements of $\mathrm{S}$ are guaranteed to have a Greatest Common Divisor, which is unique to within multiplication by the units of $S$. Since $S$ is an Euclidean Domain, a Division Algorithm can be performed in which, given $f$ and $g$, one finds $q$ (for "quotient") and $r$ (for "remainder") in $S$, with $\operatorname{deg}(r)$ strictly less than $\operatorname{deg}(g)$, to satisfy the relation: $f=g q+r$. The algorithm as well in a finite number of steps finds a Greatest Common Divisor of $f$ and g. When $(f, g)$ are coprime, the algorithm also yields the Bezout Elements, i.e. the elements $(x, y)$ which satisfy the equation: $x f+y g=1$.

The Field of Quotients of $S(s)$ is precisely the Field $R(s)$, the Field of transfer functions of arbitrary lumped linear time-invariant SISO systems, proper and stable or not. To be sure, $R(s)$ is more familiarly looked upon as the Field of Quotients of $R[s]$, as where an arbitrary rational function is expressed in "reduced" form as a quotient of coprime polynomials. But, $\mathrm{R}(\mathrm{s})$ is as well the Field of Quotients of S(s), therefore the arbitrary rational function can equally well be represented as the quotient of two stable and proper rational functions, at least one of them properly 
invertible, with no common RHP zeros -- the representation being unique to within multiplication by other proper and stable rational functions which are properly invertible and have no RHP zeros. For example:

$$
h=\frac{(s+a)(s-b)^{2}}{(s-c)(s+d)}=\frac{(s+a)(s-b)^{2} /(s+c)^{3}}{(s-c)(s+d) /(s+e)^{3}} \equiv \frac{f_{1}}{g_{1}}=\frac{(s-b)^{2} /(s+a)(s+d)}{(s-c) /(s+a)^{2}} \equiv \frac{f_{2}}{g_{2}}
$$

In (2), both $f_{1} / g_{1}$ and $f_{2} / g_{2}$ represent $h$ as a reduced-form fraction in $S$-- that is to say, each of these fractions gives a coprime factorization of $h$ over the Ring $S(s)$. Notice that the $C_{e}^{+}$zeros of $h$ (but not necessarily those zeros of $\mathrm{h}$ which are open left-half-plane) necessarily appear as zeros in the numerator of the coprime fraction; likewise, the $\mathrm{C}_{e}^{+}$poles of $\mathrm{h}$ (but not necessarily those poles which lie in the complement of $\mathrm{C}_{e}^{+}$) necessarily appear as zeros in the denominator of the coprime fraction.

$\mathbf{M}(\mathbf{S}(\mathrm{s}))$-- or, abbreviatedly, $\mathbf{M ( S )}$-- will denote the set of matrices whose entries come from S. $M(S)$ represents the set of transfer matrices of those MIMO lumped linear time-invariant systems which are, restrictively, stable and proper. $M(S(s))$, like $M(R(s))$ and $M(R[s])$, is a noncommutative Ring under the usual matrix addition and multiplication. Since S(s) is a Principal Ideal Domain, the algebra of matrices in $\mathrm{M}(S)$ is guaranteed to be analogous in all basic respects to that of the polynomial matrices. Thus, for example, the existence of Smith Forms for the elements of $M(S)$ is guaranteed. Furthermore, since $R(s)$ is the Field of quotients of $S$, coprime factorizations of the elements of $M(R(s))$ over $M(S)$ must also be achievable. Specifically, any $P(s)$ in $M\left(R(s)\right.$ ) must be expressable as a matrix fraction $P(s)=N(s) D(s)^{-1}$ with $(N, D)$ right-coprime in $M(S)$, and, equivalently, as a matrix fraction $P(s)=\bar{D}(s)^{-1} \bar{N}(s)$ with $(\bar{D}, \bar{N})$ left-coprime in $M(S)$. Vidyasagar has chosen to work with coprime fractions over the Ring of stable transfer matrices, rather than with coprime fractions over polynomial matrices, as was the style of Rosenbrock. Vidyasagar has devised algorithmic procedures for obtaining the GCD's, the Bezout elements and 
the Coprime Factorizations pertinent to the Ring $M(S)$, which closely parallel those procedures for the Ring $M(R[s])$ which Rosenbrock had earlier developed. The advantages which Vidyasagar is able to reap from having made this particular choice of an algebraic formalism can now be demonstrated.

\subsubsection{Parametrizations of Stabilizing Controllers and Stabilized Closed-Loop Performance}

Consider again the basic mulitvariable closed-loop configuration which was introduced in the previous chapter. That same configuration, re-drawn and in part re-labelled, reappears below in Figure 8.

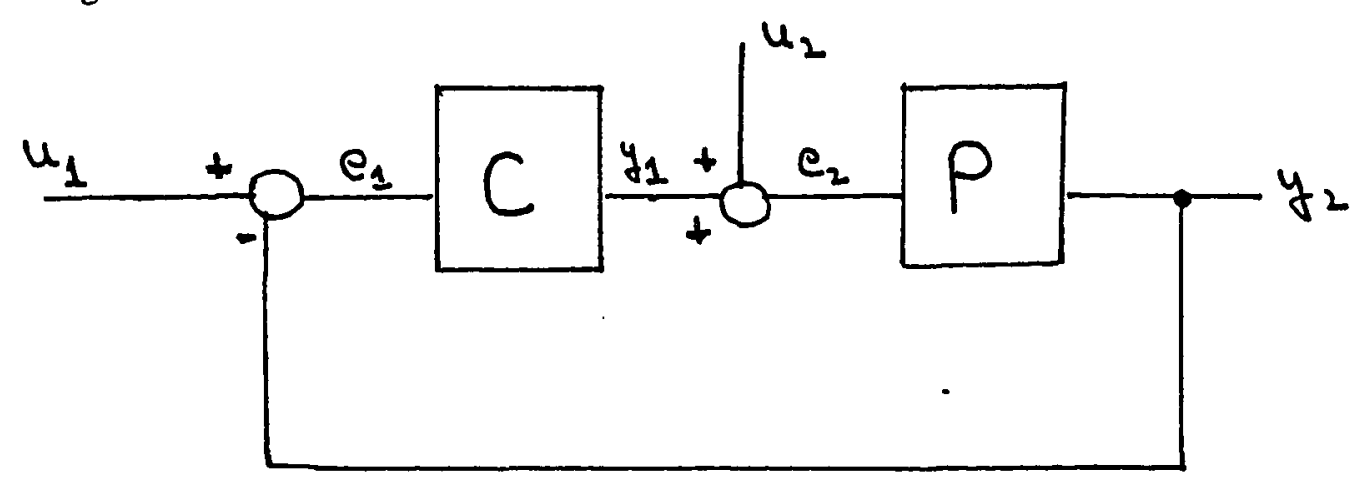

Figure 8

The transfer relationship between $\left(u_{1}^{t} u_{2}^{t}\right)^{t}$ and $\left(e_{1}^{t} e_{2}^{t}\right)^{2}$ can be expressed by:

$$
\left[\begin{array}{l}
e_{1} \\
e_{2}
\end{array}\right]=\left[\begin{array}{cc}
(I+P C)^{-1} & -(I+P C)^{-1} P \\
C(I+P C)^{-1} & I-C(I+P C)^{-1} P
\end{array}\right]\left[\begin{array}{l}
u_{1} \\
u_{2}
\end{array}\right] \equiv H(P, C)\left[\begin{array}{l}
u_{1} \\
u_{2}
\end{array}\right]
$$

$\mathrm{P}$ and $\mathrm{C}$ are, in general, members of $\mathrm{M}(\mathrm{R}(\mathrm{s}))$. The overall closed-loop system of Figure 8 is considered to be stable iff $\mathrm{H}(\mathrm{P}, \mathrm{C})$ is a matrix which belongs specifically to $M(S)$. Though the configuration is essentially symmetrical in $\mathrm{P}$ and $\mathrm{C}$, it is convenient to regard $\mathrm{P}$ as the plant and $\mathrm{C}$ 
as the compensator. The flexibility of the configuration is such that the compensator can be regarded as lying either in the forward or the feedback path. In the case that $H(P, C)$ in fact belongs to $M(S)$, the plant $P$ is said to be stabilized by the compensator $C$.

The transfer matrix between $\left(u_{1}^{t} u_{2}^{t}\right)^{z}$ and $\left(y_{1}^{t} y_{2}^{t}\right)^{t}$, designated by $W(P, C)$, is expressable as:

$$
W(P, C)=\left[\begin{array}{cc}
0 & -I \\
I & 0
\end{array}\right](I-H(P, C))
$$

By dint of (4), W(P,C) is a stability matrix if and only if $H(P, C)$ is one; in fact, the sets of poles of the two matrices are one and the same. Conventionally, the assumption is made that $\operatorname{det}(\mathrm{I}+$ $\mathrm{P}(\infty) \mathrm{C}(\infty)) \neq 0$, in order to guarantee well-posedness and regularity for the closed-loop system. Since the component systems $\mathbf{P}$ and $\mathrm{C}$ have been represented by their input-output maps, it is specifically BIBO or "extemal" stability of the closed-loop system which the stability of H(P,C) guarantees. The added assumption that both $\mathrm{P}$ and $\mathrm{C}$ have no unstable hidden modes, i.e. that each of them is both stabilizable and detectable, is therefore also conventionally made. In this event, when $P$ and $C$ are represented in MFD form by their coprime factorizations over $M(S)$, BIBO stability and complete asymptotic stability (i.e. "internal" stability) are equivalent conditions. For bounded inputs, not alone all the outputs, but as well all the intemal signals within the stable system, are then guaranteed to remain bounded.

An analogy between scalar and matrix cases helps to introduce Vidyasagar's first major algebraic result. In the scalar case, where $P=n_{p} / d_{p}$ and $C=n_{d} / d_{c}$, the transfer function from $u_{2}$ to $y_{2}$ is given, clearly, by $g=n_{p} d_{c}\left(d_{p} d_{c}+n_{p} n_{c}\right)^{-1}$. Assuming that these fractional representations for $P$ and $\mathrm{C}$ are indeed presented in the form of coprime factorizations over $\mathrm{S}, \mathrm{g}$ will be stable if and only if $\left(d_{p} d_{c}+n_{p} n_{c}\right)$ is an invertible element of $S$. A corresponding result attains in the matrix case. Starting out with the RCF $\left(N_{p}, D_{p}\right)$ of $P$ and the LCF $\left(\bar{D}_{c}, \bar{N}_{c}\right)$ of $C$, Vidyasagar shows that $\mathrm{H}(\mathrm{P}, \mathrm{C})$ is stable if and only if: 


$$
\Delta \equiv \bar{N}_{c} N_{p}+\bar{D}_{c} D_{p} \quad \text { is unimodular in } M(s)
$$

$H(P, C)$ can in fact be rewritten as:

$$
H(P, C)=\left[\begin{array}{ll}
I & 0 \\
0 & 0
\end{array}\right]+\left[\begin{array}{c}
-N_{p} \\
D_{p}
\end{array}\right] \Delta^{-1}\left[\begin{array}{ll}
\bar{N}_{c} & \bar{D}_{c}
\end{array}\right]
$$

Thus, $\Delta$ is a "retum-difference" matrix for Figure 8. Algebraically, $\operatorname{det}(\Delta(\mathrm{s}))$ is a Characteristic Polynomial of H(P,C), corresponding to the factorization of its components in terms of "polynomials" which belong to $\mathrm{S}$. As Vidyasagar suggests, in connection with the matrices over $\mathrm{S}$, "Characteristic Determinant" is a less confusing designation than "Characteristic Polynomial". The key point is that the $C_{e}^{+}$zeros of the Characteristic Determinant, det $(\Delta(s))$, are precisely the $\mathrm{C}_{\mathrm{e}}^{+}$poles of $\mathrm{H}(\mathrm{P}, \mathrm{C})$.

A second major algebraic result is the parametrization of all stabilizing compensators for P. Suppose that $\left(N_{p}, D_{p}\right)$ and $\left(\bar{D}_{p}, \bar{N}_{p}\right)$ are respectively an RCF and a LCF of $P$ and that $X$ and $Y$ satisfy the (right) Bezout Equation:

$$
\mathrm{XN}_{\mathrm{p}}+\mathrm{YD}_{\mathrm{p}}=\mathrm{I}
$$

By condition (5), $\mathrm{Y}^{-1} \mathrm{X}$ is a Left Coprime Factorization of one possible stabilizing compensator for $\mathrm{P}$. Vidyasagar goes on to show that a characterization of the set of all compensators which have the capability to stabilize $P$ is provided, subsequently, by:

$$
\operatorname{Stab}(P) \equiv\{C \in M(R(S)): H(P . C) \in M(S)\}=\left\{\left(Y-R \bar{N}_{p}\right)^{-1}\left(X+R \bar{D}_{p}\right): \operatorname{det}\left(Y-R \bar{N}_{p}\right) \neq 0\right\}
$$

In (8), $R$ is a free parameter which may range over all matrices (of the proper dimension) in $M(S)$. To yield the third major algebraic result, $H(P, C)$, the transfer matrix between $\left(u_{1}^{t} u_{2}^{t}\right)^{2}$ and 
$\left(e_{1}^{t} e_{2}^{b}\right)^{t}$, is re-written, in terms of the parameter $R$ and the $(X, Y)$ defined in (7):

$$
H(P, C)=\left[\begin{array}{cc}
I-N_{p}\left(X+R \bar{D}_{p}\right) & -N_{p}\left(Y-R \bar{N}_{p}\right) \\
D_{p}\left(X+R \bar{D}_{p}\right) & D_{p}\left(Y-R \bar{N}_{p}\right)
\end{array}\right]
$$

and $W(P, C)$, the transfer matrix between $\left(u_{1}^{t} u_{2}^{t}\right)^{t}$ and $\left(y_{1}^{t} y_{2}^{t}\right)^{t}$, is written:

$$
W(P, C)=\left[\begin{array}{cc}
D_{p}\left(X+R \bar{D}_{p}\right) & D_{p}\left(Y-R \bar{N}_{p}\right)-I \\
N_{p}\left(X+R \bar{D}_{p}\right) & N_{p}\left(Y-R \bar{N}_{p}\right)
\end{array}\right]
$$

The left-handed constructions dual to (5), (8), (9), and (10) are, of course, also obtainable. The determinant of the matrix:

$$
\tilde{\Delta} \equiv \overline{\mathrm{N}}_{\mathrm{p}} \mathrm{N}_{\mathrm{c}}+\overline{\mathrm{D}}_{\mathrm{p}} \mathrm{D}_{\mathrm{c}}
$$

defined in terms of a LCF of $P$ and a RCF of $C$, is again a Characteristic Determinant for $H(P, C)$. Thus, the unimodularity of $\tilde{\Delta}$ is an equivalent condition for the closed-loop stability of Figure 8. By this condition, the left Bezout equation:

$$
\overline{\mathrm{N}}_{\mathrm{p}} \overline{\mathrm{X}}+\overline{\mathrm{D}}_{\mathrm{p}} \overline{\mathrm{Y}}=\mathrm{I}
$$

yields $(\overline{\mathrm{X}}, \overline{\mathrm{Y}})$ as a RCF of a possible stabilizing compensator for $\mathrm{P}$. A formula which generates all the stabilizing compensators for $\mathrm{P}$ is now given in terms of the RCF provided by $7 \mathrm{~L}$, the given RCF of $\mathrm{P}$ and free matrix parameter $\mathrm{Q}$ :

$$
\operatorname{Stab}(P)=\left\{\left(\bar{X}+D_{p} Q\right)\left(\bar{Y}-N_{p} Q\right)^{-1}: Q \operatorname{in} M(S), \operatorname{det}\left(\bar{Y}-N_{p} Q\right) \neq 0\right\}
$$


The substitution of $8 \mathrm{~L}$ into expression (3) for $\mathrm{H}(\mathrm{P}, \mathrm{C})$, and into the corresponding expression for $\mathrm{W}(\mathrm{P}, \mathrm{C})$, yields:

$$
H(P, C)=\left[\begin{array}{ll}
\left.\overline{(Y}-N_{p} Q\right) \bar{D}_{p} & -\left(\bar{Y}-N_{p} Q\right) \bar{N}_{p} \\
\left(\bar{X}+D_{p} Q\right) \bar{D}_{p} & \left.I-\bar{X}+D_{p} Q\right) \bar{N}_{p}
\end{array}\right]
$$

and:

$$
W(P, C)=\left[\begin{array}{cc}
\left.\overline{(X}+D_{p} Q\right) \bar{D}_{p} & \left.\overline{(X}+D_{p} Q\right) \bar{N}_{p} \\
\left.\overline{(Y}-N_{p} Q\right) \bar{D}_{p}-I & \left.\overline{(Y}-N_{p} Q\right) \bar{N}_{p}
\end{array}\right]
$$

Note that (9L) and (10L) are written in terms of a given RCF and a given LCF of $P$, a RCF of one possible stabilizing compensator related through a left Bezout equation to the given LCF of $P$, and the free matrix parameter $Q$. The initial set of parameterizations, (9) and (10), was given in terms of the RCF and the LCF of P, a LCF of one possible stabilizing compensator related through a right Bezout equation to the given RCF, and the free matrix parameter $R$. After $\left(N_{p}, D_{p}\right),\left(\bar{D}_{p}, \bar{N}_{p}\right),(X, Y)$ and $\left.\overline{(Y}, \bar{X}\right)$ have all been posited, any given compensator in $S t a b(P)$ corresponds to one and only one stable R-matrix in the right-handed formulas (8) through (10), and to exactly one stable Q-matrix in the left-handed formulas (8L) through (10L).

Thus, the parametrizations of the set of all possible stable closed-loop performance characteristics for a given plant, i.e. of the set of all closed-loop behaviors which are achievable with a stabilizing controller, closely correspond to the parametrizations of the stabilizing compensators. Since the free parameters, $R$ and $Q$, have entered into $H(P, C)$ and $W(P, C)$ in a useful -specifically, affine -- way, these parametrizations already provide a starting point for the design of controllers by algebraic methods for the linear time-invariant multivariable plants. Stabilization is, generally speaking, only the most basic requirement which the compensation of a given plant must meet. In specific cases, further types of regulation are most likely also to be required -- e.g. tracking, disturbance-rejection, decoupling. In such contexts, the payoff that goes with 
Vidyasagar's peculiar choice of an algebraic formalism can now be stated. For having expressed the transfer matrix of the plant as a coprime fraction over the Ring of stable proper rational matrices, this approach to Control System Synthesis displays at all stages of an algebraic design procedure the maximal design freedom consistent with the maintainance of stability. This design freedom then stands to be exploited, with the tracking, disturbance-rejection and other regulation capabilities of the feedback system in view.

The above results thus provide an algebraic basis for design procedures which can be used on finite-dimensional linear time-invariant multivariable plants. A number of Vidyasagar's specific synthesis techniques will be examined in Chapter IV, where they will be adapted to the design of controllers for the multiple-time-scale systems.

\subsection{THE TOPOLOGICAL FORMALISM}

The "robustness" of a property of a given system has to do with how well that property is maintained when the system is continuously varied or perturbed, i.e. how well the property is maintained throughout some "neighborhood" of the nominal system. Topological considerations thus enter into Automatic Control when the issue of robustness of stabilization is to be addressed. Vidyasagar directs full and explicit attention to the topological preliminaries which must be covered before this issue can be precisely defined and rigorously analyzed.

\subsubsection{Topologization of the Rings $S(\mathrm{~s})$ and $M(S(s))$}

The set $\mathrm{S}(\mathrm{s})$ is already known to be a Ring with respect to addition and multiplication of rational functions, and it is clearly also a vector space with repect to the aforementioned addition 
and multiplication by scalars over either Real or Complex Fields. Now consider the following function defined on $\mathrm{S}(\mathrm{s})$ :

$$
\|\| \|_{s}: S \rightarrow R^{+}: f \rightarrow \sup _{\operatorname{Re}(s) \geq 0}|f(s)|
$$

The function $|\mathrm{I}| I_{\mathrm{S}}$ in fact satisfies the criteria for a norm, and so is suitable for converting $S$ into a topological space. By the Maximum Modulus Theorem, it further holds that:

$$
\|\mathrm{f}\| \|_{s}=\sup _{w \in R}|f(j w)|
$$

Thus, in the $\|\mathrm{I}\|_{\mathrm{s}}$-norm two stable SISO plants are "close" (i.e. $\|f-g\|_{s}$ is small) if and only if their frequency-responses compare at all frequencies.

In a space whose topology is norm-determined, all the basic topological determinations follow directly from the specification of the norm. Firstly, an open sphere, or e-neighborhood of $\mathrm{f}$ in $\mathrm{S}$ is defined by:

$$
B(f, e) \equiv\left\{g \text { in } S:|| f-g \mid \|_{s}<e, e>0\right\}
$$

A base for the norm topology is specified by the class of open spheres:

$$
\{B(f, e): f \text { in } S, e>0\}
$$

The open sets are, by definition, arbitrary unions of finite intersections of sets of the above types. Consequently, a set $A \subseteq S$ is open if and only if for all $f$ in $A$ there exists an e such that $B(f, e) \subseteq$ A. A useful topological finding is that the set of units is an open set in the normed linear space $(S,|||| S)$. 
Since a multiplication operation is defined on $S$, and since ||$f_{1} f_{2}\left|I_{S} \leq\right|\left|f_{1}\right| I_{S}|| f_{2} \mid I_{S}$ for all $\left(f_{1}, f_{2}\right)$ in $S$, the $1 / I_{S}$ norm actually gives to $S(s)$ the still stronger structure of a Normed Algebra. Furthermore, since addition on $S$ (i.e. the function: $S \times S \rightarrow S:(f, g) \rightarrow(f+g)$ ) and multiplication on $S$ (i.e. the function: $S \times S \rightarrow S:(f, g) \rightarrow f g$ ), and as well inversion of the set of units $U$ of $S$ (i.e. the function: $\left.U \rightarrow U: u \rightarrow u^{-1}\right)$, all are continuous operations, $\left(S, \| I I_{S}\right)$ has the structure of a Topological Ring as well. In short, the space $\left(S, I I \|_{S}\right)$ is endowed with highly articulable topological as well as algebraic structure.

The set $M(S)$ likewise becomes a Topological Ring, when it is equipped with the topology induced by the norm:

$$
\|\left.||\right|_{M(S)}: M(S) \rightarrow R^{+}: P \rightarrow \sup _{\operatorname{Re}(s) \geq 0}|| P(s)||_{2}
$$

In (15), \|\|$_{2}$ denotes the matrix norm on $\mathrm{C}^{\mathrm{pxq}},\|\mathrm{A}\|_{2} \equiv$ largest singular value of $\mathrm{A}$. This particular matrix norm has the significance of Operator Norm on $C^{\mathrm{pxq}}$ derived from the Euclidean norms on $C^{p}$ and $C^{q}$, and is also known in Functional Analysis as the "infinity norm":

$$
\|P\|_{\infty}=\sup _{u \text { in } L_{2}} \frac{\|\mathrm{Pu}\|_{2}}{\|u\|_{2}}=\sup _{w \text { in } R}\{\text { largest singular value of } P(j w)\}
$$

The norm (15) is thus induced, when the matrix is regarded as an operator on the space of square-integrable functions. In a physical interpretation, the operator is a transformation between finite energy input-output signal pairs, and the infinity norm is the maximum energy of the operator's response to any possible unit-energy signal.

In a highly fortuitous development, the norm-topology defined on $M(S)$ is shown to be precisely the product topology on $\mathrm{S}^{\mathrm{pxq}}$ (for appropriate dimensions $\mathrm{p}$ and $\mathrm{q}$ ) induced by the previously defined topology on $\mathrm{S}$. The base for this product topology is then specifiable as the class of 
all possible pxq products of open spheres in $\left(\mathrm{S},\|\|_{\mathrm{S}}\right)$. Consequently, the following useful property can be used: a sequence of matrices $\left(\mathrm{P}^{\mathrm{k}}\right)$ converges in the $\| \mathrm{I} \mathrm{I}_{\mathrm{M}(\mathrm{S})}$-norm to matrix $\mathrm{P}$ if and only if, componentwise, $\left(p_{\mathrm{ij}}^{\mathrm{k}}\right)$ (for each $(\mathrm{i}, \mathrm{j})$ ) converges to $\mathrm{p}_{\mathrm{ij}}$ in the given topology on $\mathrm{S}$. In short, the spaces $\left(\mathrm{S}, \| \mathrm{I} \mathrm{I}_{\mathrm{S}}\right)$ and $\left(\mathrm{M}(\mathrm{S}), \mathrm{I} \| \mathrm{I}_{\mathrm{M}(\mathrm{S})}\right)$ have the most excellent topological correspondence that could be prescribed for one another.

\subsubsection{Graph Topology on the Ring of Arbitrary Rational Matrices, $M(R(s)$ )}

On the broader set $R(s)$, the $\|/\|_{s}$-norm would not be well-defined, since any element of the set-difference $R(s)-S(s)$ "blows-up" in the closed right-hand-plane. Likewise, the $M(s)$-norm would not be well-defined on the broader set $M(R(s))$. Therefore, in venturing to topologize these larger sets, it is necessary to proceed more circumspectly than before.

The topology with which Vidasagar endows the larger set $M(R(s))$ will be called the Graph Topology. Characteristically for his version of the Fractional-Representation Approach, he proceeds in terms of the coprime factorizations over $M(S)$ of the arbitrary $P$ in $M(R(s))$. In the Graph Topology, an e-neighborhood of $\mathbf{P}$, or open sphere about $\mathbf{P}$, is defined by:

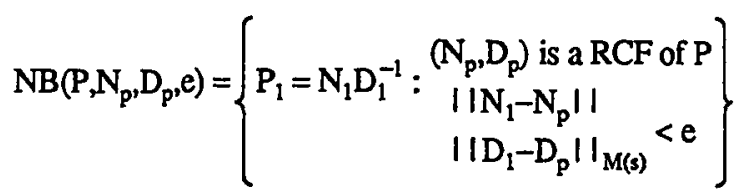

Consequently, as $\mathrm{P}$ is varied over all the elements (of the appropriate dimension) of $\mathrm{M}(\mathrm{R}(\mathrm{s})$ ), as $\left(N_{p}, D_{p}\right)$ is varied over all possible RCF's of $P$, and as $e>0$ is varied up to the point at which $\left(N_{1}, D_{1}\right)$ ceases to be a right-coprime pair, a base for the Graph Topology on $M(R(s))$ is described. The Graph Topology is now, abstracly, defined. (An analogous dual construction using the 
LCF's of $P$ is, of course, also possible; not surprisingly, it yields an equivalent topology on $\mathrm{M}(\mathrm{R}(\mathrm{s}))$.)

The Graph Topology is not itself a normed topology; the set which it topologizes remains less well structured than the normed linear space $\left(M(S), \|\left.||\right|_{M(s)}\right)$. Still, thanks to the possibility of obtaining the requisite coprime factorizations in (17), the Graph Topology on $M(R(s)$ ) is yet an appropriate extension of the normed topology on S. In the sense of the definition (17), a small perturbation of an unstable plant in $M(R(s))$ is referred to the simultaneous small perturbations of two corresponding stable plants -- which can be gauged in the normed topology on $M(S)$. With this observation, the payoff of Vidyasagar's having worked with coprime factorizations over stable rational functions in leading to a "natural" topology for the general class of lumped linear time-invariant MIMO systems becomes apparent.

The most significant results which Vidyasagar shows to follow from his definition of the Graph Topology are:

(i) Convergence in the Graph Topology can be specified in terms of convergence in the norm topology. Specifically, a sequence $\left(P_{i}\right)$ of plants in $M(R(s))$ converges to plant $P$ if and only if there exist RFC's $\left(\left(N_{i}, D_{j}\right)\right)$ of $P_{i}$ and $(N, D)$ of $P$ with $\left(N_{i}\right)$ converging to $N$ and $\left(D_{i}\right)$ converging to $\mathrm{D}$ in the norm topology on $\mathrm{M}(\mathrm{s})$.

(ii) Continuity at the point $\lambda_{0}$ of the function $P$ from an arbitrary topological space $\Lambda$ into $M(R(s))$ can be specified in terms of mappings into $M(s)$. Specifically, the function P: $\Lambda \rightarrow$ $\mathrm{M}(\mathrm{R}(\mathrm{s})): \lambda \rightarrow \mathrm{P}_{\lambda}$ is continuous at $\lambda_{0}$ if and only if there exist a family of RCF's $\left(\left(N_{\lambda}, D_{\lambda}\right)\right)$ of $P_{\lambda}$ such that each of the functions $N: \Lambda \rightarrow M(S): \lambda \rightarrow N_{\lambda}$ and $D: \Lambda \rightarrow M(S)$ : $\lambda \rightarrow D_{\lambda}$ is continuous at $\lambda_{0}$.

(iii) $M(S)$ is an open subset of $M(R(S)$ ) in the Graph Topology. On the subset $M(S)$ of $M(R(s))$, the Graph Topology and the norm topology are in fact equivalent. 
(iv) On the set-difference $M(R(s))-M(S)$, the Graph Topology fails to be the product topology on $R(s)^{p \times q}$, for the appropriate $p$ and $q$, generated by the Graph Topology on $R(s)$. However, by (iii), the Graph Topology must be a product topology when it is restricted to $M(S)$ alone. Likewise, $M(R(s))-M(S)$ with the Graph Topology fails to be a Topological Ring. Nevertheless, by (iii), M(S) with the Graph Topology is a Topological Ring.

The Graph of $\mathbf{P}$ is defined in the literature as the set of all possible stable input-output pairs associated with the operator $P$. If $\left(N_{p}, D_{p}\right)$ is an RCF of $P$, this notion can be formalized as:

$$
\text { Graph }(P) \equiv\left\{\left(N_{p} z, D_{p} z\right): z \text { is a Laplace Transformed element of } L_{2}^{q}[0, \infty)\right\}
$$

By result (i) above: $\left(\mathrm{P}_{\mathrm{i}}\right)$ converges to $\mathrm{P}$ in the Graph Topology if and only if (Graph $\left(\mathrm{P}_{\mathrm{i}}\right)$ ) converges to Graph $(P)$ in the transform-domain correspondent to $L_{2}^{+q}[0, \infty)$. In other words, two plants are close if and only if their Graphs are close. From this finding the Graph Topology assumes its name. Results (i) through (iv) jointly substantiate the Graph's Topology's claim to being the most natural and best possible extension to the class.of arbitrary plants of a normed topology which is strictly defined for the class of stable plants alone. It still remains to be shown that the Graph Topology is in fact useful: (a) in enabling the issue of robustness of stabilization to be discussed, and (b) in actually enhancing the design of robustly stabilizing controllers.

\subsubsection{The Issue of Robust Stabilizability}

Suppose now that a controller needs to be designed for a variable or imprecisely-known plant. For the sake of specificity, we let $\left(\mathrm{P}_{0}, \mathrm{C}\right)$ represent the nominal plant-compensator pair, and we allow that in the course of operation $\left(P_{0}, C\right)$ might be perturbed into $\left(P_{f}, C\right)$. For the stabilization of $P_{0}$ by $C$ to be deemed meaningfully robust, it is required: (i) that the pair $\left(P_{f}, C\right)$ remain 
stable, and as well (ii) that the set of perturbed performance-characteristics, $\mathrm{H}\left(\mathrm{P}_{\mathrm{f}}, \mathrm{C}\right)$, remain close to the nominal performance-characteristics, $\mathrm{H}\left(\mathrm{P}_{0}, \mathrm{C}\right)$, with the variations in the plant having affected the performance of the closed-loop system in a duly continuous manner. These notions are formalized by Vidyasagar in the definition that compensator $C$ in Stab $\left(\mathrm{P}_{\lambda_{0}}\right)$ robustly stabilizes the family of plants $\left(P_{\lambda}\right)$ iff (i) there exists a Neighborhood $N$ of $\lambda_{0}$ in the parameter space $\Lambda$ such that $C$ is in $S t a b\left(P_{\lambda}\right)$ for all $\lambda$ in $N$, and (ii) $H\left(P_{\lambda}, C\right)$, considered as a function from $\Lambda$ into $M(R(s))$, is continuous at the point $\lambda=\lambda_{0}$. In this formulation of the definition of Robust Stabilizability, the parameter space is required to be itself a topological space. Without any very significant loss of generality, $\Lambda$ can be taken, conventionally, as the subset $[0, \infty)$ of the Real line under the usual topology.

The key question to be asked is: given the family of plants $\left(P_{\lambda}\right)$ with $\lambda$ in some subset $N$ of $\Lambda$, can a robustly stabilizing compensator for this family be designed? With the following Theorem, Vidyasagar provides an answer to this question:

Theorem 1 [32,p241]: Suppose $\lambda \rightarrow P_{\lambda}$ is a function mapping a first countable topological space into $M(R(s))$ :

(i) Suppose that the function $\lambda \rightarrow P_{\lambda}$ is continuous at $\lambda=\lambda_{0}$ when $M(R(s))$ is equipped with the Graph Topology. Let $C$ be any element of Stab $\left(P_{\lambda}\right)$. Then, there exists a neighborhood $N$ of $\lambda_{0}$ such that $C$ is in Stab $\left(P_{\lambda}\right)$ for all $\lambda$ in $\mathrm{N}$, and moreover $\mathrm{H}\left(\mathrm{P}_{\lambda}, \mathrm{C}\right)$ is continuous (in $\mathrm{M}(\mathrm{S})$ ) as a function of $\lambda$ at $\lambda_{0}$.

(ii) Conversely, suppose there exists a $C$ in Stab $\left(P_{\lambda}\right)$ for all $\lambda$ in some neighborhood $N$ of $\lambda_{0}$, and that $H\left(P_{\lambda}, C\right)$ is continuous (in $M(S)$ ) as a function of $\lambda$ at $\lambda_{0}$. Then, the function $\lambda \rightarrow P_{\lambda}$ is continuous when $M(R(s))$ is equipped with the Graph Topology.

This important Theorem states that if and only if $\mathrm{P}$ is continuous in the Graph Topology at the point $\lambda_{0}$, will some (in fact, any) compensator in Stab $\left(\mathrm{P}_{\lambda_{0}}\right)$ be robustly stabilizing within some family of perturbations of the nominal plant. By the contrapositive to the "only if" part of this statement, if $P$ fails to be continuous at $\lambda_{0}$, then either (i) there exists no compensator in Stab $\left(P_{\lambda_{0}}\right)$ 
which has the stabilizing effect throughout some neighborhood $\mathrm{N}$ of $\lambda_{0}$, or (ii) the resulting closed-loop responses, as $\lambda$ varies throughout $\mathrm{N}$, will be discontinuously different. The "if" and "only if" parts of the Theorem, taken jointly, justify the Graph Topology's claim to being the weakest topology on $\mathrm{M}(\mathrm{R}(\mathrm{s})$ ) in which continuity makes feedback-stabilization robust against plant perturbations. Indeed, Theorem 1 gives to the property of continuity in the Graph Topology a natural and potentially useful interpretation in terms of the capability of designing robustly stabilizing compensators.

Theorem 1 can also be extended to a version which allows simultaneous variation in plant and compensator. If e (living in topological space A) represents the variable or uncertain plant parameter and $b$ (living in topological space $B$ ) represents the variable compensator parameter, the product space $A \times B$ can now play the role of $\Lambda$ in Theorem 1. The plant is then represented by the function $P: \Lambda \rightarrow M(R(s)):(e, b) \rightarrow P_{e}$, and the compensator by the function $C: \Lambda \rightarrow$ $M(R(s)):(e, b) \rightarrow C_{b}$. Under this construction, Theorem 1 states that if and only if $P$ and $C$ are both continuous at $\lambda_{0}$ (i.e. if and only if $P$ is continuous at $e_{0}$ and $C$ is continuous at $b_{0}$ ) will there exist a neighborhood $\mathrm{N}$ of $\lambda_{0}$ in the product space throughout which stability, together with continuity of the closed-loop response, is maintained. The neighborhood $\mathrm{N}$ denotes joint neighborhoods $N_{A}$ of $P_{c_{b}}$ and $N_{B}$ of $C_{b_{B}}$ such that for all perturbations of the plant through $N_{A}$ there exist corresponding satisfactorily stabilizing b-dependent compensators in $\mathbf{N}_{\mathbf{B}}$. In this sense alone are plant and compensator uncertainties to be understood as "coupled".

It follows from this extended version of Theorem 1 that if $P_{e}$ fails to be continuous at $e_{0}$ then no such coupled b-dependent family of compensators is to be found. However, by the original statement of the Theorem, if $\mathrm{P}$ is continuous at $\mathrm{e}_{0}$ then any fixed, i.e. b-independent, controller in $\operatorname{Stab}\left(\mathrm{P}_{\mathrm{e}_{0}}\right)$ performs the requisite task of robust stabilization. The extended version of Theorem 1 thus leads to a surprising and felicitous result. If a family of coupled compensators can be designed to robustly stabilize a given plant, the robust stabilization can equally be achieved 
through one fixed controller. For the purpose of achieving robust stabilization of a variable or uncertain plant, either a single compensator can be found to perform the task or else the task cannot be performed at all -- strictly speaking, a family of stabilizing compensators is never needed.

\subsubsection{Topology of the Explicit Singular Perturbations in the Frequency Domain}

Armed with the topological preliminaries which have just been covered, attention is now returned to the (explicit) singularly perturbed system. For convenience, that system is once again presented:

$$
\begin{gathered}
{\left[\begin{array}{l}
\dot{x} \\
\dot{z}
\end{array}\right]=\left[\begin{array}{cc}
A_{11} & A_{12} \\
A_{21} / e & A_{22} / e
\end{array}\right]\left[\begin{array}{l}
x \\
z
\end{array}\right]+\left[\begin{array}{c}
B_{1} \\
B_{2} / e
\end{array}\right] u=A(e)\left[\begin{array}{l}
x \\
z
\end{array}\right]+B(e) u} \\
y=\left[C_{1} C_{2}\right]\left[\begin{array}{l}
x \\
z
\end{array}\right] \equiv C\left[\begin{array}{l}
x \\
z
\end{array}\right]
\end{gathered}
$$

The reduced-order model of (19) is, once again, given by:

$$
\begin{gathered}
\dot{x}=\left(A_{11}-A_{12} A_{22}^{-1} A_{21}\right) x+\left(B_{1}-A_{12} A_{22}^{-1} B_{2}\right) u \equiv A_{0} x+B_{0} u \\
y=\left(C_{1}-C_{2} A_{22}^{-1} A_{21}\right) x-C_{2} A_{22}^{-1} B_{2} u \equiv C_{0} x+D_{0} u
\end{gathered}
$$

$P_{e}$, denoting the transfer matrix of system (19), is given by:

$$
P_{e}=C(S I-A(e))^{-1} B(e)
$$

And $\mathrm{P}_{0}$, denoting the transfer matrix of $(20)$, is given by:

$$
P_{0}=C_{0}\left(s I-A_{0}\right)^{-1} B_{0}+D_{0}
$$

As in earlier discussions, system (19) is spoken of as a singular perturbation off system (20). As well, $P_{c}$ is spoken of as a singular perturbation off $P_{0}$. Precisely, (19) represents a family of systems -- one particular member of the family corresponds to any specific value taken by the 
perturbation parameter. Following the sense of the discussion in earlier chapters, we say that the singularly perturbed system (19) is stabilized if all members of the family $\left(P_{e}\right)$, for e within some neighborhood $\left[0, \mathrm{e}^{*}\right)$ of $\mathrm{e}=\mathbf{0}$, are simultaneously stabilized by a given controller.

On the face of it, the problem of stabilizing the singularly perturbed system bears similarity to the problem of robustly stabilizing the variable or uncertain plant. Reflection on the precise definition of Robust Stabilization shows that the two are not equivalent problems, however. Stabilization of the singularly perturbed system is less stringent in requirement than robust stabilization of the variable plant. For the plant $P_{0}$ to be robustly stabilized, two conditions must be satisfied, the first of which alone is sufficient to ensure the associated singularly perturbed system $P_{e}$ 's being stabilized. By this token, the previous discussion, for having provided a necessary and sufficient topological condition for the robust stabilizability of (20), at the same time provides a sufficient topological condition for the stabilizability of (19): if the function $P_{e}$ is continuous in the Graph Topology at $e=0$, then any compensator which stabilizes the plant $\mathrm{P}_{0}$ will simultaneously stabilize all the members of a family of plants, $\left(\mathrm{P}_{\mathrm{e}}\right)$, living within a sufficiently small neighborhood of $\mathrm{P}_{0}$.

Unfortunately, the above condition is not always useful. That is to say, whether in a given case $P_{e}$ is in fact continuous at $e=0$ in the Graph Topology may not be an easy matter to determine. Since the Graph Topology is not a product topology on $M(R(s))$, the convergence of a sequence of matrices in this topology cannot be related in any simple way to the convergence of its component subsequences. The continuity of the matrix-valued function $P_{e}$ at $e=0$ neither implies nor is implied by the continuity at $e=0$ of the scalar-valued component functions $p_{i j}(e)$. The possibilities for computing continuity and convergence are made still more slight because $M(R(s))$ is not a Topological Ring. That is to say, $\left(P_{e}\right)$ 's converging to $P_{0}$ and $\left(Q_{e}\right)$ 's converging to $Q_{0}$ do not together imply $\left(P_{e}+Q_{e}\right)$ 's converging to $P_{0}+Q_{0}$. Besides addition, neither multiplication nor inversion is guaranteed to be continuous on $M(R(s))$. 
The Graph Topology indeed provides a coherent theoretical framework for the analysis of robust stabilizability and for the study of singular perturbations in the frequency domain. However, its usefulness to guarantee the stabilizability of given singularly perturbed systems in practical cases is adversely affected by the difficulty of concretely applying its notions of continuity and convergence. This serious drawback notwithstanding, continuity in the Graph Topology can practically be ascertained in several identifiable special cases, which are pertinent to the structure of the singularly perturbed system. Reapproaching the stabilization of the singularly perturbed system from the vantage point of these special cases, Vidyasagar is able to achieve a number of useful limited results.

\subsubsection{Strictly Proper Controllers for Singularly Perturbed Systems with Stable Fast Dynamics}

Vidyasagar's first special result concerning the design of controllers for the singularly perturbed system is given by Theorem 2:

Theorem 2 [32,p253]: Assuming that $A_{22}$ in (19) is both invertible and stable and that $\left(A_{0}, B_{0}, C_{0}\right)$ in (20) is both stabilizable and detectable, if $C_{2}\left(s I-A_{22}\right)^{-1} B_{2}=0$, then $P_{e}$ is continuous in the Graph Topology at $e=0$.

Under the above hypotheses (which, significantly, include open-loop stability of the system's fast dynamics), in the special case that either $B_{2}$ or $C_{2}$ is null, i.e. in the case that in (19) the fast modes are completely decoupled either from the input or from the output, any compensator in $\operatorname{Stab}\left(\mathrm{P}_{0}\right)$ stabilizes the singularly perturbed system $P_{e}$ for all values of the parameter in some neighborhood of $\mathrm{e}=\mathbf{0}$.

Vidysagar's second special result pertaining to the stabilization of the singularly perturbed system which has open-loop-stable fast dynamics is given as Theorem 3: 
Theorem 3 [32,p259]: Under the assumptions of Theorem 2, if $\mathrm{C}$ is a strictly proper controller in Stab $\left(\mathrm{P}_{0}\right)$, then (i) $\mathrm{C}$ is in Stab $\left(\mathrm{P}_{e}\right)$ for sufficiently small $\mathrm{e}$, and (ii) $\mathrm{H}\left(\mathrm{P}_{e}, \mathrm{C}\right)$ converges to $\mathrm{H}\left(\mathrm{P}_{0}, \mathrm{C}\right)$ uniformly over all compact subsets of the closed Right Half Plane.

When $\mathrm{C}$ is a strictly proper controller, the above hypotheses guarantee, alongside C's stabilizing the singularly perturbed system $\mathrm{P}_{e}$, a certain continuity condition on the family's resultant closed-loop response. The Theorem falls short of strictly guaranteeing the closed-loop response's continuity in the Graph Topology, since the convergence condition described by (ii) is not guaranteed uniformly over the entire extended Right Half Plane. Condition (ii) provides, nonetheless, a result which is eminently worthwhile in its own right.

Theorems 2 and 3, which Vidyasagar casts within a framework of topological analysis, compare with earlier results which were obtained through State Variable analysis by $\mathrm{H}$. K. Khalil [14]. The latter results were reviewed in Chapter I in connection with the time-domain analysis of the explicit singularly perturbed system. In Khalil's finding, the condition $\mathrm{B}_{2} \mathrm{~F}_{2} \mathrm{C}_{2}=0$ ensured basically the same results as guaranteed by the present Theorems of Vidyasagar. $F_{2}$ was a parameter associated with the dynamic compensator in Khalil's feedback scheme. This dynamic compensator ( (21) of Chapter I ) had, specifically, the transfer function:

$$
C(s)=F_{1}\left(s I-\left(G_{1}+G_{3} F_{1}\right)\right)^{-1}\left(G_{2}+G_{3} F_{2}\right)+F_{2}
$$

In the time-domain, when $F_{2}$ is a null matrix there is no static feedback from the output to the input of the plant; in the frequency-domain, $F_{2}$ 's being a null matrix is tantamount to the strict properness of the compensator.

A recent paper by J. O'Reilly [23] presents the following schematic representation of dynamic compensator control of the explicit singularly perturbed system: 


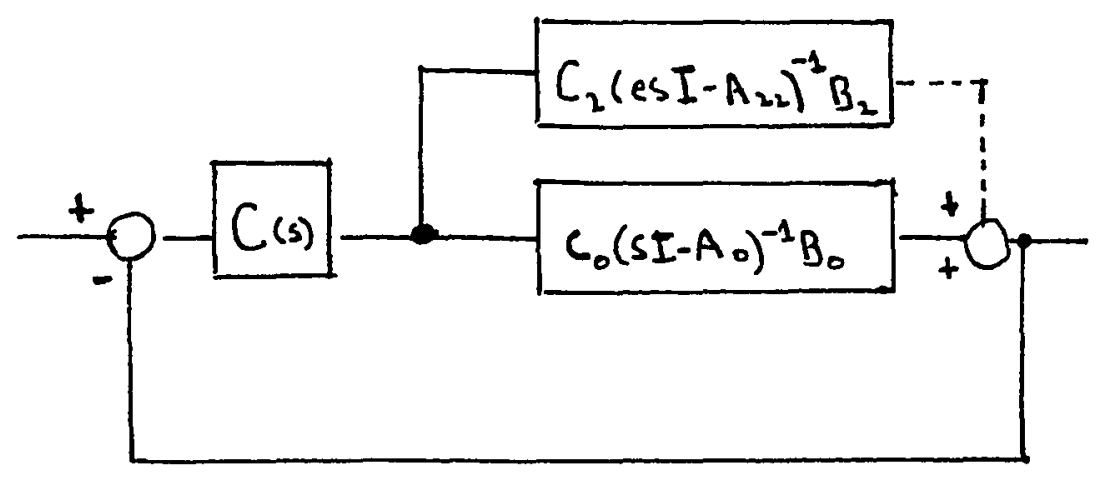

Figure 9

In Figure $9, \mathrm{P}_{\mathrm{e}}$ is represented by the parallel combination of two lower-order parameterindependent systems, refiecting the approximate frequency-domain decomposition:

$$
P_{e}(s)=C_{0}\left(s I-A_{0}\right)^{-1} B_{0}+C_{2}\left(e s I-A_{22}\right)^{-1} B_{2}+O(e)
$$

When $\mathrm{C}(\mathrm{s})$ is not strictly proper, the loop around $\mathrm{P}_{\mathrm{c}}$ 's high frequency dynamics may be regarded as effectively closed. In such a case, any high-frequency mode which is both controllable and observable with respect to $\left(A_{22}, B_{2}, C_{2}\right)$ will be shifted from its open-loop location as an eigenvalue of $\mathrm{A}_{22} / \mathrm{e}$ to the approximate closed-loop location of the corresponding eigenvalue of $\left(A_{22}+B_{2} F_{2} C_{2}\right) / e$. Thus, when the compensator is not strictly proper, the jointly controllable and observable fast modes have the capability to destabilize the closed-loop system, even should these modes be themselves in fact open-loop-stable. But when $C(s)$ is strictly proper, the loop around the high-frequency dynamics remains, effectively, open. In such a case, no fast mode of $P_{\varepsilon}$ will be affected by the output feedback to any greater extent than $O(e)$. Consequently, all the open-loop-stable high-frequency modes remain within some region of stability after the basic feedback loop is closed.

That strict propemess of the controller is necessary as well as sufficient to guarantee the result of Theorem 3, one further finding of Vidyasagar's, is stated in Theorem 4: 
Theorem 4 [33,p147]: Given a system of type (20), with transfer matrix $P_{0}$, if $C$ is a nonstrictly proper controller in Stab $\left(P_{0}\right)$,

there exists a triple (A, B, C) comprising a system of type (19), with $A_{22}$ a stability matrix, of which $P_{c}$ is the transfer matrix and $P_{0}$ the reduced-order model, such that for each $e>0, P_{e}$ is destabilized by $C$.

The quadruple $\left(A_{0}, B_{0}, C_{0}, D_{0}\right)$ which constitutes a given system of type (20) is correspondable to different (A, B, C) triples in a system of type (19). Any one of these triples now counts as a possible family of singular perturbations off the given quadruple. By Theorem 4, when a given system of type (20) is compensated with a nonstrictly proper feedback controller, there exists a family of singular perturbations off the system, one in fact whose high-frequency dynamics are open-loop stable, which this controller nevertheless destabilizes.

Theorem 4 bears import for a standard application of the singular-perturbational method, in which fast modes are not alone neglected or unmodelled but actually unknown. That is to say, the reduced-order model from which fast modes have been removed represents the sole knowledge of the system which is at the designer's disposal, rather than an artful choice of design-model made in the light of wider knowledge. Theorem 4 points out a hazard connected with the use of nonstrictly proper controllers in such an application. Theorem 3, on the other hand, permits strictly proper controllers to be designed in these applications, provided that the assumption of open-loop stability of the plant's (unspecified) high-frequency parasitics is warranted. 


\section{CHAPTER IV}

\section{DESIGN PROCEDURES FOR THE MULTIPLE-TIME-SCALE SYSTEMS}

\subsection{OVERVIEW OF THE ARGUMENT OF THE DISSERTATION}

In the previous Chapter, a particular formalism imposed upon the Ring of rational matrices in the work of M. Vidyasagar [29-34] has revealed the topological structure of the explicit singular perturbations in the frequency domain. Vidyasagar's results have permitted strictly proper reduced-order dynamic output controllers to be designed for the explicit singularly perturbed system only when that system's high-frequency dynamics either are known to be or may be presumed to be stable. Vidyasagar has shown specifically that when the system's fast modes are unknown and cannot be presumed stable, or when they are known specifically not to be stable, his design cannot be guaranteed to work.

In Chapter I's review of the pertinent literature on singular perturbations in the time domain, a paper by $\mathrm{H} . \mathrm{K}$. Khalil [14] presented the current state-of-the-art in the design of dynamic output controllers for the explicit singularly perturbed system. With regard to the design of such controllers for this system, Vidyasagar and Khalil have come to identical results. Both these authors restrict their discussion to the system which is explicitly singularly perturbed, and neither of them brings into consideration the possibility of designing dynamic controllers for the singularly perturbed plant which has instabilities in its fast or "parasitic" dynamics as well as in 
the slow or "normal" modes.

In Chapter I it was shown how, by state-feedback methods, controllers designed separately for the reduced-order "slow" and "fast" subsystems can be combined into composite controllers for such two-fold-unstable explicitly singularly perturbed plants. The decentralized design of the separate-and-composite type, which brings both slow and fast subsystems into play, represents a more comprehensive controller strategy, suited to a less restrictive application, than that considered by Vidyasagar and Khalil. Yet, to this writer's knowledge, no design strategy for the singularly perturbed system involving separate-and-composite dynamic output controllers has so far been reported in the literature.

In the interest of identifying a broader class of linear autonomous systems which display multiple-time-scale behavior, the implicit singularly perturbed system has also been introduced. In Chapter I, the implicit singularly perturbed system was characterized and studied from the time-domain point of view, and in Chapter II from the frequency-domain viewpoint. In these respective contexts, a pair of very deep theoretical results were brought to light -- the results of Coderch, Willsky, Sastry and Castanon [3] on the approximation, by a set of reduced-order parameter-independent State Transition Matrices, of the multiple-time-scale State Transition Matrix $\exp (\mathrm{A}(\mathrm{e}) \mathrm{t})$, and $\mathrm{D}$. W. Luse's [18-21] results conceming the approximation, by a set of lower-dimensional parameter-independent Transfer Matrices, of the multiple-frequency-scale Transfer Matrix $H(s, e)$. Specifically, Luse has proved a Theorem pertaining to the location of closed-loop poles in a feedback configuration of two-frequency-scale systems of the more general type in relation to the poles of corresponding lower-order parameter-independent closed-loop systems.

The present Chapter opens with a re-examination of Luse's Closed Loop Theorem. The Theorem is here seen to provide, in outline, a comprehensive separate-and-composite design strategy, involving dynamic output controllers, for the broadest class of linear autonomous multiple 
time scale systems. The body of the chapter then adapts a number of specific synthesis techniques derivative from the algebraic formalism of Vidyayagar for the pivotal role of converting the Theorem's implicitly suggested design strategy into a set of concrete and implementable design procedures. In Chapters I through III, through exposition and analysis of the theoretical work of the above-mentioned authors, the various pieces of the design puzzle have been honed. The present Chapter features the distinctively original work of the dissertation; here, the pieces are fused into a novel and practical synthesis.

\subsection{REEXAMINATION OF LUSE'S CLOSED-LOOP THEOREM}

Figure 10a below represents an actual feedback system, each of whose component systems has the two-frequency-scale property. Figures $10 \mathrm{~b}$ and $10 \mathrm{c}$ are respectively the lowfrequency and high-frequency reduced-order approximating models for the given feedback system.

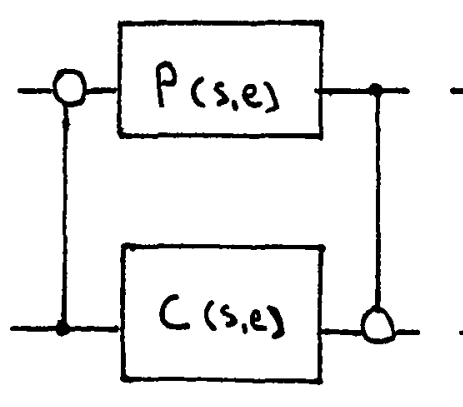

Figure 10a

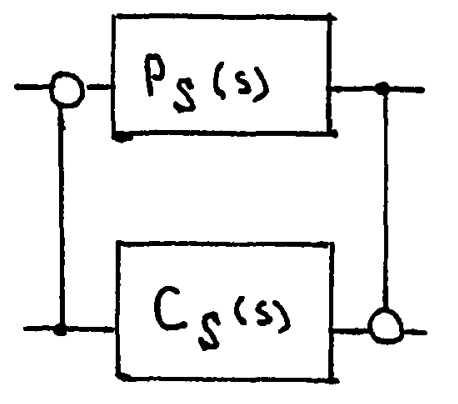

Figure 10b

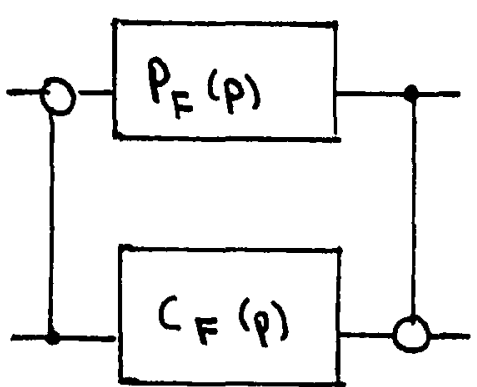

Figure 10c

Luse's Closed-Loop Theorem is here restated with reference to the above Figures: 
Theorem 1: Assuming that $\left(I+P_{S}(\infty) C_{S}(\infty)\right)$ and $\left(I+P_{F}(\infty) C_{F}(\infty)\right)$ are nonsingular,

all poles of the closed-loop system of Figure 10a are either within $(1+$ $0(e))$ times $\left\{\left(s_{i}\right)\right\}$ or within $(1+0(e))$ times $\left\{\left(p_{i} / e\right)\right\}$, where:

$\left\{\left(s_{\mathrm{i}}\right)\right\}$ denotes the set of poles of the closed-loop slow subsystem, Figure $10 \mathrm{~b}$, plus the lost slow poles of $\mathrm{P}(\mathrm{s}, \mathrm{e})$ and of $\mathrm{C}(\mathrm{s}, \mathrm{e})$, and:

$\left\{\left(p_{i}\right)\right\}$ denotes the set of poles of the corresponding closed-loop fast subsystem, Figure 10c, plus the lost fast poles of $\mathrm{P}(\mathrm{s}, \mathrm{e})$ and of $\mathrm{C}(\mathrm{s}, \mathrm{e})$.

An immediate Corollary to the Theorem has the form of a stability-result:

\section{Corollary 1: Under the conditions of Theorem 1,}

if slow and fast closed-loop subsystems and the sets of slow and fast lost poles are all stable,

then the closed-loop system of Figure 10a will be stable for all values of $\mathrm{e}$ in $\left(0, \mathrm{e}^{*}\right]$ for some upper bound $\mathrm{e}^{*}$.

By Luse's Theorem, the poles of the actual feedback system are, asymptotically (i.e. as the parameter approaches zero): (i) the set of open-loop lost poles of $\mathrm{P}(\mathrm{s}, \mathrm{e})$ and of $\mathrm{C}(\mathrm{s}, \mathrm{e})$, plus (ii) the set of poles of the closed-loop system depicted in Figure 10b, plus (iii) 1/e times the set of closed-loop poles in Figure 10c. By the Corollary, if the open-loop lost poles of the component systems in Figure 10a and the closed-loop poles in Figures $10 \mathrm{~b}$ and $10 \mathrm{c}$ all reside in the left half of the Complex Plane, the actual feedback system is stable at all sufficiently small values of the perturbation parameter.

It is here desired to turn the Corollary's stability result into a design principle for the given class of systems. Towards this end, it is apposite to start with the result of the Theorem and then work backwards. $\mathrm{P}(\mathrm{s}, \mathrm{e})$, in this context, represents a two-frequency-scale plant in need of regulation. The representation uses frequency-domain input-output techniques, without the avail of intemal system models for displaying possible hidden modes which might destabilize the feedback system. Therefore, it must be assumed at the outset that all lost poles of $\mathrm{P}(\mathrm{s}, \mathrm{e})$ are stable and also that its lower-order approximating models, $P_{S}(s)$ and $P_{F}(p)$, are both stabilizable and 
detectable. With these conditions met, a second system which is constructed to meet the hypotheses of the Corollary to the Theorem qualifies as a suitable stabilizing controller for $P(s, e)$.

Supposing that $C_{S}(s)$ is devised as a stabilizing and regulating compensator for $P_{S}(s)$ and that $C_{F}(p)$ is devised separately to stabilize and regulate $P_{F}(p)$, we desire to subsequently compose $\mathrm{C}_{\mathrm{S}}(\mathrm{s})$ and $\mathrm{C}_{\mathrm{F}}(\mathrm{p})$ into a single controller which satisfactorily stabilizes and regulates the twofrequency-scale system, $\mathrm{P}(\mathrm{s}, \mathrm{e})$. In order to be validated by Luse's Theorem, this composite controller must satisfy the following three conditions: (i) it must be itself a two-frequency-scale system, (ii) it must have no unstable lost poles, and (iii) its own reduced-order slow and fast approximating models must be, precisely, $\mathrm{C}_{S}(\mathrm{~s})$ and $\mathrm{C}_{\mathrm{F}}(\mathrm{p})$, In addition, the conditions $\operatorname{det}\left(I+C_{S}(\infty) P_{S}(\infty)\right) \neq 0$ and $\operatorname{det}\left(I+C_{F}(\infty) P_{F}(\infty)\right) \neq 0$ must hold, to preserve the well-posedness and the regularity of the feedback system.

Recall that the "fast" frequency variable has been scaled according to the relation, $p=e s$. Thus, the simplest composition of $C_{S}(s)$ and $C_{F}(p)$ is:

$$
C_{1}(s, e)=C_{s}(s)+C_{F}(e s)
$$

The candidate controller $\mathrm{C}_{1}$ has for its reduced-order low-frequency and high-frequency approximating models:

$$
\left.\mathrm{C}_{1}(\mathrm{~s}, 0)=\mathrm{C}_{\mathrm{S}}(\mathrm{s})+\mathrm{C}_{\mathrm{F}}(0) \quad \text { and } \quad \mathrm{C}_{1}(\mathrm{p} / \mathrm{e}), \mathrm{e}\right) \underset{\sim=0}{I}=\mathrm{C}_{\mathrm{F}}(\mathrm{p})+\mathrm{C}_{\mathrm{S}}(\infty)
$$

The compensator $\mathrm{C}_{1}$ thus fails to satisfy condition (iii). Happily, its deviation from the needed result is very simple and transparent, and the proper correction can immediately be made. We first impose as a matching condition the requirement that:

$$
\mathrm{C}_{\mathrm{S}}(\infty)=\mathrm{C}_{\mathrm{F}}(0)=\mathrm{K}
$$


In (3), $\mathrm{K}$ may be any constant Real matrix. We now compose $\mathrm{C}(\mathrm{s}, \mathrm{e})$ by:

$$
C(s, e)=C_{s}(s)+C_{F}(e s)-K
$$

The compensator $\mathrm{C}$ has for its reduced-order models:

$$
\mathrm{C}(\mathrm{s}, 0)=\mathrm{C}_{\mathrm{S}}(\mathrm{s}) \quad \text { and } \quad \mathrm{C}((\mathrm{p} / \mathrm{e}), \mathrm{e}) \underset{\sim=0}{\mathrm{I}}=\mathrm{C}_{\mathrm{F}}(\mathrm{p})
$$

The compensator $\mathrm{C}$, through satisfying the matching condition, indeed satisfies condition (iii).

The compensator $\mathrm{C}(\mathrm{s}, \mathrm{e})$ is now representable as the following parallel composition:

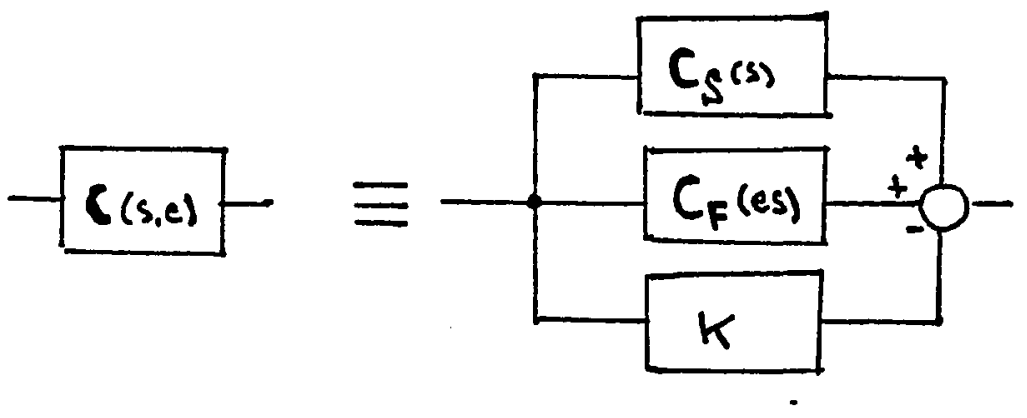

Figure 11

In a parallel composition of this sort, if no pole of any one of the component subsystems is a pole of any other component, then the composite system is guaranteed to have least order. The poles of $\mathrm{C}_{\mathrm{S}}(\mathrm{s})$ are $\mathrm{O}(1)$, and the poles of $\mathrm{C}_{\mathrm{F}}(\mathrm{es})$ are $\mathrm{O}(1 / \mathrm{e})$. These sets of poles, having distinct orders of magnitude, cannot possibly overlap. Therefore, Figure 11 indeed provides a least order representation of the candidate controller. The poles of $\mathrm{C}(\mathrm{s}, \mathrm{e})$, being precisely the set union of the poles of $C_{S}(s)$ with the poles of $C_{F}(p)$, are such that Luse's Second Criterion necessarily holds for the compensator $C(s, e)$. Provided, further, only that $C_{s}(s)$ and $C_{F}(p)$ are proper, Luse's First criterion is also satisfied. $C(s, e)$, composed out of $C_{S}(s)$ and $C_{F}(p)$ consistently with the matching condition 
(3), is indeed a two-frequency-scale system according to Luse's Criteria, and the present condition (i) as well holds. Because $\mathrm{C}(\mathrm{s}, \mathrm{e})$ has been explicitly constructed in this manner, it is represented exactly by the parallel connection in Figure 11, and it has no lost poles. $(\mathrm{P}(\mathrm{s}, \mathrm{e})$, on the other hand, in general experiences a decomposition into $\mathrm{P}_{\mathrm{S}}(\mathrm{s})$ and $\mathrm{P}_{\mathrm{F}}(\mathrm{p})$; in line with Luse's Approximation Result, it is then approximately represented by their parallel connection.) Having no lost poles, $\mathrm{C}(\mathrm{s}, \mathrm{e})$ has a fortiori no unstable lost poles (condition (ii)). $\mathrm{C}(\mathrm{s}, \mathrm{e})$ is now suitable as a controller for the two-frequency-scale plant $P(s, e)$, because it satisfies all the hypotheses of the stability Corollary to Luse's Theorem.

As stated, $\mathrm{C}_{\mathrm{S}}(\mathrm{s})$ and $\mathrm{C}_{\mathrm{F}}(\mathrm{p})$ are chosen separately to impart desirable characteristics to the closed-loop systems of Figures $10 \mathrm{~b}$ and $10 \mathrm{c}$ respectively. In a time-domain design which was reviewed in Chapter 1, the two separate matrices of state-feedback which contributed to the formation of a composite state-feedback control law could be chosen in complete independence of one another. The present separate-and-composite dynamic controller design, however, is complicated by the constraint which represents the matching-condition. In the typical application, the regulation needs of the steady-state, "slow" part of the plant are most likely to have the paramount importance. In such a case, $\mathrm{C}_{\mathrm{S}}(\mathrm{s})$ can be designed with complete freedom, and $\mathrm{C}_{\mathrm{F}}(\mathrm{p})$ later chosen subject to the constraint that $C_{F}(0)$ match $C_{S}(\infty)$. In other applications, the primary requirement might be to shape the system's transient fast dynamics in some specific way. Then, the order of the design procedure would be reversed, with the coupling-condition appearing rather as a constraint which the design of the slow controller must satisfy.

The special case in which $\mathrm{P}_{\mathrm{F}}(\mathrm{p})$ is already stable corresponds to that limited application which has already been studied in the work of Khalil and in the work of Vidyasagar. In this application, the fast controller, $\mathrm{C}_{\mathrm{F}}(\mathrm{p})$, in effect is chosen as identically zero, and the matching condition then constrains the slow controller, $C_{S}(s)$, to the requirement of strict properness -- exactly as Khalil and Vidysagar have prescribed! Luse's Theorem also dictates a complementary result for 
that application in which the slow subsystem, $P_{S}(s)$, is already stable. Here, in effect, the slow controller is chosen as identically zero, and the fast controller is thereby required by the matching condition to block the zero, or "dc", frequency. This dc-blocking controller is then guaranteed to prevent any of the jointly controllable and observable open-loop-stable slow modes from possibly destabilizing the feedback system.

Strict properness of the slow controllers and dc-blocking fast controllers can be made useful to the design also in the general case. The strategem of restricting the design in all cases to strictly proper controllers for slow subsystems and to dc-blocking controllers for fast subsystems will in fact be incorporated into the design procedures which are presently to be devised. The adoption of the strategem will ensure that the matching-condition (3) is always automatically satisfied. It provides the further mathematical convenience and economy of tying down the value of the matching-matrix to zero in every allowable design.

\subsection{IMPLEMENTATION ON THE EXPLICIT SINGULARLY PERTURBED SYSTEM}

Luse's Closed Loop Theorem applies to the implicit as well as the explicit singularly perturbed two-frequency-scale system. As promised, Vidyasagar's Fractional-Representation methods will be employed to convert the design strategy implicitly suggested by the Theorem into a set of concrete and implementable design procedures for the systems belonging to this general class. Luse has used the Rosenbrock System Matrix to represent the general two-frequencyscale system, $\mathrm{P}(\mathrm{s}, \mathrm{e})$, in terms of canonical Matrix Fraction descriptions over the Ring $\mathrm{R}[\mathrm{s}]$. He showed that such representations are in theory always achievable, and he made schematic use of these representations in furnishing the proofs for his important results, including the Closed Loop Theorem. But $\mathrm{P}(\mathrm{s}, \mathrm{e})$ needs to be representable in terms of specific coprime Matrix Fraction 
descriptions over the Ring $M(S)$, if Vidyasagar's methods are to be applicable. For a given system which is explicitly singularly perturbed, these requisite factorizations over $M(S)$ can be provided immediately and in full explicitness. Therefore, the design will first be concretized for the explicit singularly perturbed system.

\subsubsection{Obtaining Doubly Coprime Factorizations from State Space Representations}

In the special case where $\mathrm{P}(\mathrm{s}, \mathrm{e})$ derives from the state-variable description of an explicit singularly perturbed system, it has the form:

$$
P(s, e)=\left[C_{1} C_{2}\right]\left[s I-\left[\begin{array}{cc}
A_{11} & A_{12} \\
A_{21} / e & A_{22} / e
\end{array}\right]\right]^{-1}\left[\begin{array}{c}
B_{1} \\
B_{2} / e
\end{array}\right] \equiv C(s I-A(e))^{-1} B(e)
$$

The given plant is assumed, as before, to be strictly proper. The Transfer Matrices of the reduced-order approximating subsystems corresponding to (5) have, once again, the forms:

$$
P_{S}(s)=C_{o}\left(s I-A_{0}\right)^{-1} B_{0}+D_{0} \quad \text { and } \quad P_{F}(p)=C_{2}\left(p I-A_{22}\right)^{-1} B_{2}
$$

where:

$$
\begin{aligned}
& A_{0} \equiv A_{11}-A_{12} A_{22}^{-1} A_{21} \quad C_{0} \equiv C_{1}-C_{2} A_{22}^{-1} A_{21} \\
& \mathrm{~B}_{0} \equiv \mathrm{B}_{1}-\mathrm{A}_{12} \mathrm{~A}_{22}^{-1} \mathrm{~B}_{2} \quad \mathrm{D}_{\mathrm{o}} \equiv-\mathrm{C}_{2} \mathrm{~A}_{22}^{-1} \mathrm{~B}_{2}
\end{aligned}
$$

A Lemma formulated by Nett, Jacobson and Balas [22] provides those exact factorizations over M(s) pertinent to system (5) which will enable the full array of Vidyasagar's controller synthesis methods to be brought into play: 
Lemma 1 [22,p831]: Given the Transfer Matrix $P=C(s I-A)^{-1} B+E$, assuming that $(A, B, C)$ is stabilizable and detectable, choose matrices $\mathrm{K}$ and $\mathrm{L}$ which respectively make (A-BK) and (A-LC) stability matrices:

then, a RCF of $\mathrm{P}$ is given by:

$$
P=\left((C-E K)(S I-(A-B K))^{-1} B+E\right)\left(I-K(s I-(A-B K))^{-1} B\right)^{-1} \equiv N_{p} D_{p}^{-1}
$$

and a LCF is given by:

$$
P=\left(I-C(s I-(A-L C))^{-1} L\right)^{-1}\left(C(s I-(A-L C))^{-1}(B-L E)+E\right) \equiv \bar{D}_{p}^{-1} \bar{N}_{p}
$$

Furthermore, a pair $(X, Y)$ which satisfies the right Bezout Equation $X N$ $+\mathrm{YD}=\mathrm{I}$ is provided by:

$$
(X, Y)=\left(\left(K(S I-(A-L C))^{-1} L\right),\left(I+K\left(S I-(A-L C)^{-1}\right)(B-L E)\right)\right.
$$

and a pair $(\bar{X}, \bar{Y})$ which satisfies the left Bezout Equation $\bar{N} \bar{X}+\bar{D} \bar{Y}=I$ is provided by:

$$
(\bar{X}, \bar{Y})=\left(\left(K(S I-(A-B K))^{-1} L\right),\left(I+(C-E K)(s I-(A-B K))^{-1} L\right)\right)
$$

Lemma 1 in fact provides a Doubly Coprime Factorization of P. The Doubly Coprime Factorization refers, technically, to a pair of matrices $\left[\left[\begin{array}{cc}Y & X \\ -\bar{N} & \bar{D}\end{array}\right],\left[\begin{array}{cc}D & -\bar{X} \\ N & \bar{Y}\end{array}\right]\right)$ which satisfy the set of relations:

$$
\left[\begin{array}{cc}
\mathrm{Y} & \mathrm{X} \\
-\overline{\mathrm{N}} & \overline{\mathrm{D}}
\end{array}\right]\left[\begin{array}{cc}
\mathrm{D} & -\overline{\mathrm{X}} \\
\mathrm{N} & \overline{\mathrm{Y}}
\end{array}\right]=\left[\begin{array}{ll}
\mathrm{I} & 0 \\
0 & \mathrm{I}
\end{array}\right]
$$

When $R$ is any Principal Ideal Domain and $F$ is the Field of Quotients of $R$, any $P$ in $M(F)$ is guaranteed to support a Doubly Coprime Factorization over matrices in $M(R)$. The Doubly Coprime Factorization is unique in the sense that, given any RCF, $(N, D)$, and any $L C F,(\bar{D}, \bar{N})$, of $P$ and given any pair $(X, Y)$ which satisfies the right Bezout Equation in terms of $(N, D)$, there 
exists one and only one pair $(\bar{X}, \bar{Y})$ which satisfies the left Bezout Equation in terms of $(\bar{D}, \bar{N})$ and as well the specific relation $X \bar{Y}=Y \bar{X} . \quad(X, Y)$ and $(\bar{X}, \bar{Y})$ provide, in other words, respectively a LCF and a RCF of one and the same stabilizing compensator for $\mathrm{P}$.

Doubly Coprime Factorizations for $\mathrm{P}_{\mathrm{S}}(\mathrm{s})$ and $\mathrm{P}_{\mathrm{F}}(\mathrm{p})$ are immediately obtainable from the Lemma, once suitable controller-gains $\mathrm{K}_{\mathrm{s}}$ and $\mathrm{K}_{\mathrm{f}}$ and suitable observer-gains $\mathrm{L}_{s}$ and $\mathrm{L}_{\mathrm{f}}$ have been chosen in separate eigenvalue-placement problems. Specifically:

$$
\begin{aligned}
& P_{S}(s)=\left(\left(C_{0}-D_{0} K_{s}\right)\left(s I-A_{0}+B_{0} K_{s}\right)^{-1} B_{0}+D_{0}\right)\left(I-K_{s}\left(s I-A_{0}+B_{0} K_{s}\right)^{-1} B_{0}\right)^{-1} \equiv N_{s} D_{s}^{-1} \\
&=\left(I-C_{0}\left(s I-A_{0}+L_{s} C_{0}\right)^{-1} L_{s}\right)^{-1}\left(C_{0}\left(s I-A_{0}+L_{s} C_{0}\right)^{-1}\left(B_{0}-L_{s} D_{0}\right)+D_{0}\right) \equiv \bar{D}_{s}^{-1} \bar{N}_{s} \\
&\left(X_{s}, Y_{s}\right)=\left(\left(K_{s}\left(s I-A_{0}+L_{s} C_{0}\right)^{-1} L_{s}\right),\left(I+K_{s}\left(s I-A_{0}+L_{s} C_{0}\right)^{-1}\left(B_{0}-L_{s} D_{0}\right)\right)\right. \\
&\left(\bar{X}_{s}, \bar{Y}_{s}\right)=\left(\left(K_{s}\left(s I-A_{0}+B_{0} K_{s}\right)^{-1} L_{s}\right),\left(I+\left(C_{0}-D_{0} K_{s}\right)\left(s I-A_{0}+B_{0} K_{s}\right)^{-1} L_{s}\right)\right) \\
& P_{F}(p)=\left(C_{2}\left(p I-A_{22}+B_{2} K_{f}\right)^{-1} B_{2}\right)\left(I-K_{f}\left(p I-A_{22}+B_{2} K_{f}\right)^{-1} B_{2}\right)^{-1} \equiv N_{f} D_{f}^{-1} \\
&=\left(I-C_{2}\left(p I-A_{22}+L_{f} C_{2}\right)^{-1} L_{f}\right)^{-1}\left(C_{2}\left(p I-A_{22}+L_{f} C_{2}\right)^{-1} B_{2}\right) \equiv \bar{D}_{f}^{-1} \bar{N}_{f} \\
& \\
& \\
&\left(X_{F}, Y_{F}\right)=\left(\left(K_{f}\left(p I-A_{22}+L_{f} C_{2}\right)^{-1} L_{f}\right),\left(I+K_{f}\left(p I-A_{22}+L_{f} C_{f}\right)^{-1} B_{2}\right)\right) \\
&\left(X_{F}, Y_{F}\right)=\left(\left(K_{f}\left(p I-A_{22}+B_{2} K_{f}\right)^{-1} L_{f}\right),\left(I+C_{2}\left(p I-A_{22}+B_{2} K_{f}\right)^{-1} L_{f}\right)\right)
\end{aligned}
$$

\subsubsection{Stabilizing Controllers and Closed-Loop Designs for the Reduced-Order Subsystems}

The parametrizations of the stabilizing compensators and the achievable closed-loop designs for the slow and fast subsystems in terms of free matrix parameters immediately follow from the Doubly Coprime Factorizations, (12) and (13). The set of stabilizing compensators for the slow reduced-order model is given by:

$$
\begin{aligned}
\operatorname{Stab}\left(\mathrm{P}_{S}(\mathrm{~s})\right) & =\left\{\left(\mathrm{Y}_{s}-\mathrm{R}_{s} \bar{N}_{s}\right)^{-1}\left(\mathrm{X}_{s}+\mathrm{R}_{s} \overline{\mathrm{D}}_{s}\right): \mathrm{R}_{s} \text { in } \mathrm{M}(\mathrm{s}), \operatorname{det}\left(\mathrm{Y}_{s}-\mathrm{R}_{s} \bar{N}_{s}\right) \neq 0\right\} \\
& =\left\{\left(\bar{X}_{s}+\mathrm{D}_{s} \mathrm{Q}_{s}\right)\left(\overline{\mathrm{Y}}_{s}-\mathrm{N}_{s} \mathrm{Q}_{s}\right)^{-1}: \mathrm{Q}_{s} \operatorname{in} \mathrm{M}(\mathrm{s}), \operatorname{det}\left(\overline{\mathrm{Y}}_{s}-\mathrm{N}_{s} \mathrm{Q}_{s}\right) \neq 0\right\}
\end{aligned}
$$


And the set of stabilizing compensators for the fast subsystem is given by:

$$
\begin{aligned}
\operatorname{Stab}\left(\mathrm{P}_{\mathrm{F}}(\mathrm{p})\right) & =\left\{\left(\mathrm{Y}_{\mathrm{f}}-\mathrm{R}_{\mathrm{f}} \overline{\mathrm{N}}_{\mathrm{f}}\right)^{-1}\left(\mathrm{X}_{\mathrm{f}}+\mathrm{R}_{\mathrm{f}} \overline{\mathrm{D}}_{\mathrm{f}}\right): \mathrm{R}_{\mathrm{f}} \operatorname{in} \mathrm{M}(\mathrm{s}), \operatorname{det}\left(\mathrm{Y}_{\mathrm{f}}-\mathrm{R}_{\mathrm{f}} \overline{\mathrm{N}}_{\mathrm{f}}\right) \neq 0\right\} \\
& =\left\{\left(\overline{\mathrm{X}}_{\mathrm{f}}+\mathrm{D}_{\mathrm{f}} \mathrm{Q}_{\mathrm{f}}\right)\left(\overline{\mathrm{Y}}_{\mathrm{f}}-\mathrm{N}_{\mathrm{f}} \mathrm{Q}_{\mathrm{f}}\right)^{-1}: \mathrm{Q}_{\mathrm{f}} \text { in } \mathrm{M}(\mathrm{s}), \operatorname{det}\left(\overline{\mathrm{Y}}_{\mathrm{f}}-\mathrm{N}_{\mathrm{f}} \mathrm{Q}_{\mathrm{f}}\right) \neq 0\right\}
\end{aligned}
$$

The set of achievable closed-loop responses, $\left\{W\left(P_{S}(s), C(s)\right): C(s)\right.$ in Stab $\left.\left(P_{S}(s)\right)\right\}$, is now characterizable in terms of either of the free affine parameters, $R_{s}(s)$ or $Q_{s}(s)$ :

$$
\begin{aligned}
& W\left(P_{S}, R_{s}\right)=\left[\begin{array}{ll}
D_{s}\left(X_{s}+R_{S} \bar{D}_{S}\right) & D_{s}\left(Y_{S}-R_{S} \bar{N}_{S}\right)-I \\
N_{S}\left(X_{S}+R_{S} \bar{D}_{s}\right) & N_{S}\left(Y_{S}-R_{S} \bar{N}_{S}\right)
\end{array}\right] \\
& W\left(P_{S}, Q_{S}\right)=\left[\begin{array}{ll}
\left(X_{s}+D_{s} Q_{s}\right) \bar{D}_{s} & \left(X_{s}+D_{s} Q_{S}\right) \bar{N}_{s} \\
\left(\bar{Y}_{s}-N_{s} Q_{s}\right) \bar{D}_{s}-I & \left(\bar{Y}_{s}-N_{s} Q_{S}\right) \bar{N}_{s}
\end{array}\right]
\end{aligned}
$$

Likewise, the set, $\left\{W\left(P_{F}(p), C(p)\right): C(p)\right.$ in $\left.\operatorname{Stab}\left(P_{F}(p)\right)\right\}$, is characterizable in terms of either of the free affine parameters $R_{f}(p)$ or $Q_{f}(p)$ :

$$
\begin{aligned}
& W\left(P_{F}, R_{f}\right)=\left[\begin{array}{ll}
D_{F}\left(X_{f}+R_{f} \bar{D}_{f}\right) & D_{F}\left(Y_{f}-R_{f} \bar{N}_{f}\right)-I \\
N_{F}\left(X_{f}+R_{f} \bar{D}_{f}\right) & N_{F}\left(Y_{f}-R_{f} \bar{N}_{f}\right)
\end{array}\right] \\
& W\left(P_{F} Q_{f}=\left[\begin{array}{cc}
\left(\bar{X}_{f}+D_{f} Q_{f}\right) \bar{D}_{f} & \left(\bar{X}_{f}+D_{f} Q_{f}\right) \bar{N}_{f} \\
\left(\bar{Y}_{f}-N_{f} Q_{f}\right) \bar{D}_{f}-I & \left(\bar{Y}_{f}-N_{f} Q_{f}\right) \bar{N}_{f}
\end{array}\right]\right.
\end{aligned}
$$


In Chapter I, the compensation of system (5) through the controller-gain state-feedback matrix:

$$
K \equiv\left[K_{1} K_{2}\right]=\left[\left(I+K_{f} A_{22}^{-1} B_{2}\right) K_{s}+K_{f} A_{22}^{-1} A_{21} \quad K_{f}\right]
$$

yielded the stable State Matrix:

$$
A_{c o}=\left[\begin{array}{cc}
A_{11}-B_{1} K_{1} & A_{12}-B_{1} K_{2} \\
\left(A_{21}-B_{2} K_{1}\right) / e & \left(A_{22}-B_{2} K_{2}\right) / e
\end{array}\right]
$$

Analogously, the full-order observer designed for (5), implemented through the observer-gain state-feedback matrix:

$$
L \equiv\left[\begin{array}{l}
L_{1} \\
L_{2}
\end{array}\right]=\left[\begin{array}{c}
L_{s}\left(I+C_{2} A_{22}^{-1} L_{f}\right)+A_{12} A_{22}^{-1} L_{f} \\
L_{f}
\end{array}\right]
$$

produced the stabilized State Matrix:

$$
A_{o b}=\left[\begin{array}{ll}
A_{11}-L_{1} C_{1} & A_{12}-L_{1} C_{2} \\
\left(A_{21}-L_{2} C_{1}\right) / e & \left(A_{22}-L_{2} C_{2}\right) / e
\end{array}\right]
$$

The Lemma can be formally applied to system (5) itself, with the substitution of $A_{c o}$ for the Lemma's (A-BK) and of $A_{o b}$ for the Lemma's (A-LC). B and $C$ in the Lemma now represent $\left(B_{1}^{t}\left(B_{2} / e\right)^{t}\right)^{t}$ and $\left(C_{1} C_{2}\right)$ respectively. Assuming, once again, that $P$ is strictly proper, in the Lemma $\mathrm{E}$ is zero. The formal result reads:

$$
\begin{gathered}
P(s, e)=\left(C\left(s I-A_{c o}(e)\right)^{-1} B(e)\right)\left(I-K\left(s I-A_{c o}(e)\right)^{-1} \equiv N(s, e) D(s, e)^{-1}\right. \\
=\left(I-C\left(s I-A_{o b}(e)\right)^{-1} L\right)^{-1}\left(C\left(s I-A_{o b}(e)\right)^{-1} B(e)\right) \equiv \bar{D}(s, e)^{-1} \bar{N}(s, e) \\
(X(e), Y(e))=\left(\left(K\left(s I-A_{n b}(e)\right)^{-1} L\right),\left(I+K\left(s I-A_{o b}(e)\right)^{-1} B(e)\right)\right. \\
(\bar{X}(e), \bar{Y}(e))=\left(\left(K\left(s I-A_{o b}(e)\right)^{-1} L\right),\left(I+C\left(s I-A_{c o}(e)\right)^{-1} L\right)\right)
\end{gathered}
$$


The quantities corresponding to (14) through (17) which pertain directly to system (5) can also formally be obtained. We thus might consider, as an expression for the set of stabilizing compensators for $\mathrm{P}(\mathrm{s}, \mathrm{e})$ :

$$
\begin{aligned}
\operatorname{Stab}(P(s, e)) & =\left\{\left(X(e)-R_{c} \bar{N}(s, e)\right)^{-1}\left(X(e)+R_{e} \bar{D}(s, e)\right): \text { nonsingularity }\right\} \\
& =\left\{\left(\bar{X}(e)+D(s, e) Q_{e}\right)\left(\bar{Y}(e)-N(s, e) Q_{e}\right)^{-1}: \text { nonsingularity }\right\}
\end{aligned}
$$

In (23), $N(s, e), D(s, e), \bar{D}(s, e), \bar{N}(s, e), X(e), Y(e), \bar{X}(e)$ and $\bar{Y}(e)$ all derive from the formal Doubly Coprime Factorization (22), and $R_{e}$ and $Q_{e}$ are required to be stable matrices over the Field of rational functions with coefficients analytic in the perturbation parameter. From (23), an expression for $\{\mathrm{W}(\mathrm{P}(\mathrm{s}, \mathrm{e}), \mathrm{C}(\mathrm{s}, \mathrm{e})): \mathrm{C}(\mathrm{s}, \mathrm{e})$ in $\mathrm{Stab}(\mathrm{P}(\mathrm{s}, \mathrm{e}))\}$ could next formally be written as an affine function of $R_{e}(s)$ or $Q_{e}(s)$. Even with the parametrizing matrices $R_{e}$ and $Q_{e}$, very reasonably, restricted to rational functions over the Real Field, $\left\{\mathrm{W}\left(\mathrm{P}(\mathrm{s}, \mathrm{e}), \mathrm{R}_{\mathrm{e}}\right)\right\}$ and $\left\{\mathrm{W}\left(\mathrm{P}(\mathrm{s}, \mathrm{e}), \mathrm{Q}_{\mathrm{e}}\right)\right\}$ contain parameter-dependent expressions -- which are, more likely than not,untransparent, nonfactorizable and otherwise ill-conditioned for numerical treatment. In the present design, the exact structure is underlain not by $\left\{\mathrm{W}\left(\mathrm{P}(\mathrm{s}, \mathrm{e}), \mathrm{R}_{\jmath}\right)\right\}$ or $\left\{\mathrm{W}\left(\mathrm{P}(\mathrm{s}, \mathrm{e}), \mathrm{Q}_{\jmath}\right)\right\}$ but by $\left\{\mathrm{W}\left(\mathrm{P}(\mathrm{s}, \mathrm{e}), \mathrm{R}_{\mathrm{s}}, \mathrm{R}_{\mathrm{f}}\right)\right\}$ or $\left\{\mathrm{W}\left(\mathrm{P}(\mathrm{s}, \mathrm{e}), \mathrm{Q}_{\mathrm{s}}, \mathrm{Q}_{\mathrm{f}}\right)\right\}$ (or, possibly, by $\left\{\mathrm{W}\left(\mathrm{P}(\mathrm{s}, \mathrm{e}), \mathrm{R}_{\mathrm{s}}, \mathrm{Q}_{\mathrm{f}}\right)\right\}$ or by $\left.\left\{\mathrm{W}\left(\mathrm{P}(\mathrm{s}, \mathrm{e}), \mathrm{Q}_{s} \cdot \mathrm{R}_{\mathrm{f}}\right)\right\}\right)$. The latter again contain e-dependent expressions; furthermore, the parametrizing matrices which appear in them, because they pertain to the factorizations of the subsystems rather than to the factorization of $\mathrm{P}(\mathrm{s}, \mathrm{e})$ itself, do not in this case even enter into them in affine ways. Thanks to Luse's Theorem, however, provided that the value of the perturbation-parameter remains sufficiently small, the poles of $\mathrm{W}\left(\mathrm{P}(\mathrm{s}, \mathrm{e}), \mathrm{R}_{s}, \mathrm{R}_{\mathrm{f}}\right)$ or $\mathrm{W}\left(\mathrm{P}(\mathrm{s}, \mathrm{e}), \mathrm{Q}_{s}, \mathrm{Q}_{\mathrm{f}}\right)$ (or of $\mathrm{W}\left(\mathrm{P}(\mathrm{s}, \mathrm{e}), \mathrm{R}_{s}, \mathrm{Q}_{\mathrm{f}}\right)$ or $\mathrm{W}\left(\mathrm{P}(\mathrm{s}, \mathrm{e}), \mathrm{Q}_{s}, \mathrm{R}_{\mathrm{f}}\right)$ ) are sufficiently well approximable through the poles of the matrices $W\left(P_{s}, R_{s}\right), W\left(P_{s}, Q_{s}\right), W\left(P_{f}, R_{f}\right)$, and $W\left(P_{f}, Q_{f}\right)$-- which are given, quite conveniently, in (16) and (17). In consequence, the parameter-independent Doubly Coprime Factorizations of the reduced-order 
models suffice for the present design purposes; the intractable and problematic parameterdependent factorizations related to $\mathrm{P}(\mathrm{s}, \mathrm{e})$ itself need not receive any further consideration.

\subsubsection{The Constraint of Strict Propemess and the dc-Blocking Requirement}

By the requirement of strict properness, infinity is to be a blocking zero of $\mathrm{C}_{\mathrm{s}}(\mathrm{s})$, and by the dc-blocking requirement, zero is to be a blocking zero of $\mathrm{C}_{\mathrm{F}}(\mathrm{p})$. As defined in Chapter II, $\mathrm{s}_{1}$ is a blocking zero of $\mathrm{G}(\mathrm{s})$ iff $\mathrm{G}\left(\mathrm{s}_{1}\right)=0$. Algebraically, any extended open right half plane blocking zero of $G(s)$ was shown necessarily to appear as a zero of the smallest invariant factor in the Smith form of the numerator matrix of a coprime factorization of $G(s)$ over $M(R[s])$ or over $M(s)$. In the quest to incorporate into Vidyasagar's algebraic framework the constraints of strict properness for $\mathrm{C}_{S}(\mathrm{~s})$ and the dc-blocking property for $\mathrm{C}_{\mathrm{F}}(\mathrm{p})$, the significance of the blocking zeros of the general Transfer Matrix, G(s), will now be more deeply explored,

A starting point for this study is the following very general property which pertains to Matrix Topological Rings:

Lemma 2 [32,p111]: When $R$ is a Principal Ideal Domain, if $(A, B)$ is a pair in $M(R)$ with the same number of columns, $A$ being square and nonsingular, it follows that the set:

$$
V=\{Q \text { in } M(R): \operatorname{det}(A-Q B) \neq 0\}
$$

is a dense open subset of $M(R)$ in the product topology induced by any topology on $\mathrm{R}$ in which the set of units is open and inversion is a continuous operation.

Lemma 2 guarantees almost every element of $M(R)$ to belong to the set $V$. If a given element $Q_{1}$ happens not to lie within this set, arbitrarily small perturbations of $Q_{1}$ nonetheless do belong to it. With the stronger condition that $(\mathrm{A}, \mathrm{B})$ is a right-coprime pair in $\mathrm{M}(\mathrm{R})$, Vidyasagar obtains the 
following still stronger topological result, alongside an algebraic result:

Lemma 3 [32.p97]: With all quantities denoted as in Lemma 2, if (A,B) is a right-coprime pair in $M(R)$, then if $b$, representing the smallest invariant factor in the Smith form of B, is not a unit, it follows that:

(i) the complement of $V$ in $M(R)$ is empty, and

(ii) $\operatorname{det}((A-Q B), b)$ is a coprime pair in $R$ for all $Q$ in $M(R)$.

Because the matrix $\mathrm{Y}$ which pertains to a Doubly Coprime Factorization of $\mathrm{P}$ is nonsingular and has the same dimension as the column size of $\overline{\mathrm{N}}_{\mathrm{p}}$, by Lemma 2 the set:

$$
\left\{\mathrm{R} \text { in } \mathrm{M}(\mathrm{S}): \operatorname{det}\left(\mathrm{Y}-\mathrm{R} \overline{\mathrm{N}}_{\mathrm{p}}\right) \text { is nonsingular }\right\}
$$

is a dense open subset of $\mathrm{M}(\mathrm{S})$. For this reason, the nonsingularity condition which contrains the otherwise free choice of the parameter in the expressions for the stabilizing compensators for $P$ is satisfied, in the general case, by almost all stable matrices of the proper dimension. Because, furthermore, the pair $\left(\mathrm{Y}_{\mathrm{N}} \overline{\mathrm{N}}_{\mathrm{p}}\right)$ pertaining to the Doubly Coprime Factorization is demonstrably a right-coprime pair, Lemma 3 permits $S t a b(P)$ to be characterized altogether without the nonsingularity constraints in those cases where the smallest invariant factor of $\mathrm{N}_{\mathrm{p}}$ is not a unit, i.e. in cases where $P$ has one or more blocking zeros. The zeros of $\operatorname{det}\left(Y-R \bar{N}_{p}\right)$ are the poles of the Compensator and the zeros of $\mathrm{b}$ are the blocking zeros of the Plant. Statement (ii) of Lemma 3 thus guarantees each $C$ in $S t a b(P)$ to be analytic at each blocking zero of $P$. Since the strictly proper plant has a blocking zero at infinity, every compensator which stabilizes this plant must be analytic at infinity, i.e. it must be proper.

The following Theorem from Control System Synthesis extends the previous two Lemmas towards a practical result. This time Vidyasagar's Proof is presented along with the Theorem's statement, since it features a constructive procedure which will serve in the following design procedures: 
Theorem 2 [32,p114]: Given plant $P$ in $M(R(s)$ ) and given points ( $\left.s_{1}, \ldots, s_{n}\right)$ in $C_{e}^{+}$, none of them a pole of $P$,

there exists a stabilizing compensator for $\mathrm{P}$ which has blocking zeros at the points $\left(s_{1}, \ldots, s_{n}\right)$.

P:

Proof: At the outset, we assume the Doubly Coprime Factorization for

$$
\left[\left[\begin{array}{cc}
\mathrm{Y} & \mathrm{X} \\
-\overline{\mathrm{N}} & \overline{\mathrm{D}}
\end{array}\right],\left[\begin{array}{ll}
\mathrm{D} & -\overline{\mathrm{X}} \\
\mathrm{N} & \overline{\mathrm{Y}}
\end{array}\right]\right)
$$

Select an element $f$ in $S$ such that: (i) $f$ has a zero at each of the $\left(s_{i}\right)$, and (ii) $(f, \operatorname{det}(\bar{D}))$ are coprime in $S$. Let $d$ henceforth represent $\operatorname{det}(\bar{D})$.

By coprimeness, there exist elements $a_{1}$ and $b_{1}$ in $S$ such that: $a_{1} d+b_{1} f=1$. As well, there exist elements $a$ and $b$ in $S$ such that $a d+b f=x$, for all $x$ in $S$. That is to say, for all $x$ in $S$, there exists an element a in $S$ such that $f$ divides ( $x-a d)$. Now, select a matrix $A$ in $M(S)$ such that each element of the matrix (X-Ad) is divisible by $\mathrm{f}$.

Define the matrix $R$ by: $R \equiv-A \bar{D}^{\text {adj }}$. Then, $R \bar{D}=-A d$, and hence $(X+$ $R \bar{D})$ is a multiple of $f$. Since $f$ by construction vanishes at each of the $\left(s_{i}\right),(X+$ $R \bar{D})\left(s_{i}\right)=0$ for $i=1, \ldots, n$. DNN. Thus:

By the Doubly Coprime Factorization, $\mathrm{XN}+\mathrm{YD}=\mathrm{I}$, and also, $\overline{\mathrm{N}} \mathrm{D}=$

$$
(X+R \bar{D}) N+(Y-R \bar{N}) D=I
$$

and consequently $C=(Y-R \bar{N})^{-1}(X+R \bar{D})$ is a stabilizing compensator for $P$. $C$ is, specifically, a controller for $P$ which vanishes at each of the $\left(s_{i}\right)$ !

The compensator $\mathrm{C}$ is in fact well defined. Since $(X-R \bar{D})\left(s_{i}\right)=0,(Y-R \bar{N})\left(s_{j}\right) D\left(s_{i}\right)=I$. Consequently, for each of the $\left(s_{j}\right)$, $\operatorname{det}\left((Y-R N)\left(s_{j}\right)\right) \neq 0$, thus $(Y-R \bar{N})$ is nonsingular in $M(R(s))$.

By Theorem 2, every plant with no pole at infinity has a compensator with a blocking zero at infinity -- i.e. every proper plant has a strictly proper compensator. As well, every plant with no pole at zero has a dc-blocking compensator. Each of these results will be put to use in the design procedures related to Luse's Theorem, since the $\mathrm{P}_{\mathrm{S}}(\mathrm{s})$ which calls for a strictly proper com- 
pensator is (in general, nonstrictly) proper, and the strictly proper $\mathrm{P}_{\mathrm{F}}(\mathrm{p})$ for which a dc-blocking compensator is to be designed is guaranteed to have no pole at $\mathrm{p}=0$.

Clearly, the set of functions which have zeros at each of the $\left(s_{i}\right)$ is an Ideal in the Ring or Algebra S(s), and likewise the set of matrices which have blocking zeros at each of the $\left(s_{i}\right)$ is an Ideal in the Ring or Algebra $M(S(S))$. The Ideal in $S$ will be represented by $I$ and the Ideal in $M(S)$ will be represented by $M(I)$. The element $f$ chosen in the constructive procedure in the proof of Theorem 1 is, moreover, a generator of I. Using these facts, it is possible to obtain expressions for the set of all ( $\mathrm{s}_{\mathrm{i}}$-blocking compensators which the constructive procedure in the proof of Theorem 1 might have yielded. Letting $\hat{X}$ and $\hat{Y}$ now stand, repectively, for that specific $(X+R \bar{D})$ and $(Y-R \bar{N})$ which the procedure has first generated, it is submitted that:

$$
\left\{C \text { in Stab }(P): C\left(s_{i}\right)=0\right\}=\left\{(\hat{Y}-R \bar{N})^{-1}(\hat{X}+R \bar{D}): R \text { in } M(I)\right\}
$$

In (25), the set of stabilizing compensators for $P$ which have blocking zeros at each of the $\left(s_{i}\right)$ is characterized; the nonsingularity condition is permissibly omitted from the statement of this result.

\section{Proof of Claim (25):}

If $C$ is a member of the right-hand set in (25), then (since $M(I)$ is a subset of $M(S)$ ) by the basic result on the parametrization of the stabilizing compensators, $C$ is a stabilizing compensator for $P$ Since $\hat{X}$ and $R$ both belong to $M(I)$, it follows $(M(I)$ being an Ideal) that $(\hat{X}+R \bar{D})$ as well belongs to $M(I)$; consequently, $\mathrm{C}$ has a blocking zero at each of the $\left(\mathrm{s}_{\mathrm{i}}\right)$.

Conversely, if $\mathrm{C}$ is a stabilizing compensator for $\mathrm{P}$ which has blocking zeros at each of the $\left(s_{\mathfrak{i}}\right)$, then (since $\hat{X} N+\hat{Y} D=I$ ) by the basic result on the parametrization of the stabilizing compensators this compensator has the form $(\hat{\mathrm{Y}}-\mathrm{R} \overline{\mathrm{N}})^{-1}(\hat{\mathrm{X}}+\mathrm{R} \overline{\mathrm{D}})$ for some $\mathrm{R}$ in $M(S)$. $(\hat{\mathrm{X}}+\mathrm{R} \overline{\mathrm{D}})$, being the numerator-matrix in a coprime factorization over $M(S)$ of the $\left(s_{i}\right)$-blocking compensator, clearly belongs to $M(I)$. $\hat{X}$ by construction belongs to $M(I)$, therefore (since $M(I)$ is an Ideal) $R \bar{D}$ as well belongs to $M(I)$, and also $R \bar{D} \bar{D}^{\mathrm{adj}}$, or Rd. Now, $d$ is known 
to have no zeros among the $\left(s_{i}\right)$, since the $\left(s_{i}\right)$ may not be poles of $P$. Therefore, the $R$ in $M(S)$ which characterizes $C$ actually belongs to $M(\mathrm{I})$.

$\mathbf{I}_{\infty}$ shall henceforth represent the Ideal of strictly proper functions in $S$, and $I_{0}$ the Ideal in $S$ of functions which vanish at $s=0$. I, unsubscripted, will henceforth be used in contexts where either $\mathrm{I}_{\infty}$ or $\mathrm{I}_{0}$ might be designated. By (25), the set of all strictly proper stabilizing compensators for $P_{S}(s)$ is given by:

$$
\begin{aligned}
\left\{\mathrm{C}_{\mathrm{S}}(\mathrm{s}) \text { in } \operatorname{Stab}\left(\mathrm{P}_{\mathrm{S}}(\mathrm{s})\right): \mathrm{C}_{\mathrm{S}}(\infty)=0\right\} & =\left\{\left(\mathrm{Y}_{2}-\mathrm{R}_{2} \overline{\mathrm{N}}_{s}\right)^{-1}\left(\mathrm{X}_{2}+\mathrm{R}_{2} \mathrm{D}_{s}\right): \mathrm{X}_{2} \text { in } \mathrm{M}\left(\mathrm{I}_{\infty}\right) ; \mathrm{N}_{s} \mathrm{X}_{2}+\mathrm{D}_{s} \mathrm{Y}_{2}=\mathrm{I} ; \mathrm{R}_{2} \text { in } \mathrm{M}\left(\mathrm{I}_{\infty}\right)\right\} \\
& =\left\{\left(\overline{\mathrm{X}}_{2}+\mathrm{D}_{2} \mathrm{Q}_{2}\right)\left(\overline{\mathrm{Y}}_{2}-\mathrm{N}_{s} \mathrm{Q}_{2}\right)^{-1}: \overline{\mathrm{X}}_{2} \text { in } \mathrm{M}\left(\mathrm{I}_{\infty}\right) ; \overline{\mathrm{X}}_{2} \overline{\mathrm{N}}_{\mathrm{s}}+\overline{\mathrm{Y}}_{2} \overline{\mathrm{D}}_{s}=\mathrm{I} ; \mathrm{Q}_{2} \text { in } \mathrm{M}\left(\mathrm{I}_{\infty}\right)\right\}
\end{aligned}
$$

Likewise, the set of all $d c$-blocking stabilizing compensators for $\mathrm{P}_{\mathrm{F}}(\mathrm{p})$ is given by:

$$
\begin{aligned}
\left\{C_{F}(p) \text { in } \operatorname{Stab}\left(P_{F}(p)\right): C_{F}(0)=0\right\} & =\left\{\left(Y_{3}-R_{3} \bar{N}_{f}\right)^{-1}\left(X_{3}+R_{3} \bar{D}_{f}\right): X_{3} \text { in } M\left(I_{0}\right) ; N_{f} X_{3}+D_{f} Y_{3}=I ; R_{3} \text { in } M\left(I_{0}\right)\right\} \\
& =\left\{\left(\bar{X}_{3}+D_{f} Q_{3}\right)\left(\bar{Y}_{3}-N_{f} Q_{3}\right)^{-1}: \bar{X}_{3} \text { in } M\left(I_{0}\right) ; \bar{X}_{3} \bar{N}_{f}+\bar{Y}_{3} \bar{D}_{f}=I ; Q_{3} \text { in } M\left(I_{0}\right)\right\}
\end{aligned}
$$

In the design procedures which are about to be presented, the elements $\left(\mathrm{X}_{3}, \mathrm{Y}_{3}\right)$ and $\left(\overline{\mathrm{X}}_{3}, \overline{\mathrm{Y}}_{3}\right)$ in $(27)$ will be obtained through the constructive procedure in the Proof of Theorem 1. The $\mathrm{X}$ and $\overline{\mathrm{X}}$ which are obtained directly from Lemma 1 are already strictly proper, therefore the $\left(\mathrm{X}_{3}, \mathrm{Y}_{\mathrm{s}}\right)$ and $\left(\overline{\mathrm{X}}_{3}, \overline{\mathrm{Y}}_{3}\right)$ in $(12)$ can be immediately applied as the elements $\left(\mathrm{X}_{2}, \mathrm{Y}_{2}\right)$ and $\left(\overline{\mathrm{X}}_{2}, \overline{\mathrm{Y}}_{2}\right)$ in $(26)$.

The sets of possible closed-loop designs for the reduced-order subsystems, subject to the constraints of strict properness on the slow compensator and dc-blocking property on the fast compensator are now given, in the right-handed version, by: 


$$
\begin{aligned}
& W\left(P_{S}, R_{2}\right)=\left[\begin{array}{ll}
D_{s}\left(X_{2}+R_{2} \bar{D}_{s}\right) & D_{s}\left(Y_{2}-R_{2} \bar{N}_{s}\right)-I \\
N_{s}\left(X_{2}+R_{2} \bar{D}_{s}\right) & N_{s}\left(Y_{2}-R_{2} \bar{N}_{s}\right)
\end{array}\right] \\
& W\left(P_{F}, R_{3}\right)=\left[\begin{array}{ll}
D_{f}\left(X_{3}+R_{3} \bar{D}_{f}\right) & D_{f}\left(Y_{3}-R_{3} \bar{N}_{f}\right)-I \\
N_{f}\left(X_{3}+R_{3} \bar{D}_{f}\right) & N_{f}\left(Y_{3}-R_{3} \bar{N}_{f}\right)
\end{array}\right]
\end{aligned}
$$

and, in the left-handed version, by:

$$
\begin{aligned}
& W\left(P_{S}, Q_{2}\right)=\left[\begin{array}{cc}
\left(\bar{X}_{2}+D_{s} Q_{2}\right) \bar{D}_{s} & \left(\bar{X}_{2}+D_{s} Q_{2}\right) \bar{N}_{s} \\
\left(\bar{Y}_{2}-N_{s} Q_{2}\right) \bar{D}_{s}-I & \left(\bar{Y}_{2}-N_{s} Q_{2}\right) \bar{N}_{s}
\end{array}\right] \\
& W\left(P_{F}, Q_{3}\right)=\left[\begin{array}{cc}
\left(\bar{X}_{3}+D_{f} Q_{3}\right) \bar{D}_{f} & \left.\bar{X}_{3}+D_{f} Q_{3}\right) \bar{N}_{f} \\
\left(\bar{Y}_{3}-N_{f} Q_{3}\right) \bar{D}_{f}-I & \left(\bar{Y}_{3}-N_{f} Q_{3}\right) \bar{N}_{f}
\end{array}\right]
\end{aligned}
$$

The performance characteristics in (28) derive immediately from (16) and (17), with $\left(X_{2}, Y_{2}, R_{2}\right)$ substituted for $\left(\mathrm{X}_{3}, \mathrm{Y}_{\mathrm{g}}, \mathrm{R}_{\mathrm{s}}\right)$ and with $\left(\mathrm{X}_{3}, \mathrm{Y}_{3}, \mathrm{R}_{3}\right)$ substituted for $\left(\mathrm{X}_{\mathrm{f}}, \mathrm{Y}_{\mathrm{f}}, \mathrm{R}_{\mathrm{f}}\right)$, while those in (29) derive from (16) and (17), with $\left(\overline{\mathrm{X}}_{2}, \overline{\mathrm{Y}}_{2}, \mathrm{Q}_{2}\right)$ substituted for $\left(\overline{\mathrm{X}}_{\mathrm{s}}, \overline{\mathrm{Y}}_{\mathrm{s}}, \mathrm{Q}_{8}\right)$ and with $\left(\overline{\mathrm{X}}_{3}, \overline{\mathrm{Y}}_{3}, \mathrm{Q}_{3}\right)$ substituted for $\left(\overline{\mathrm{X}}_{\mathrm{f}}, \overline{\mathrm{Y}}_{\mathrm{f}}, \mathrm{Q}_{\mathrm{f}}\right)$. Within the present notation, the Bezout elements $\mathrm{X}_{2}$ and $\overline{\mathrm{X}}_{2}$ are strictly proper, and the parameters $R_{2}$ and $Q_{2}$ are required to be chosen strictly proper, the Bezout elements $X_{3}$ and $\bar{X}_{3}$ have the dc-blocking property, and the parameters $R_{3}$ and $Q_{3}$ are required to be chosen with the dc-blocking property. $\left(\mathrm{Y}_{2}, \overline{\mathrm{Y}}_{2}, \mathrm{Y}_{3}, \overline{\mathrm{Y}}_{3}\right)$ are, of course, the corresponding Bezout elements for $\left(\mathrm{X}_{2}, \overline{\mathrm{X}}_{2}, \mathrm{X}_{3}, \overline{\mathrm{X}}_{3}\right)$. With the attainment of formulas (26) through (29), a given plant of type (5) is concretely set up for any and all computations required for the fractional-representation-based decentralized dynamic controller design procedures making use of strictly proper controllers for slow subsystems and dc-blocking controllers for fast subsystems. 


\subsubsection{Design Procedure \#1: Stabilization}

If the mere stabilization of $P(s, e)$ is required, then any chosen $R_{2}(s)$ or any chosen $Q_{2}(s)$ in $M\left(I_{\infty o}\right)$ and any chosen $R_{3}(p)$ or any chosen $Q_{3}(p)$ in $M\left(I_{0}\right)$ may be substituted into formulas (26) through (29), and the problem is forthwith solved. The null matrix belongs both to $M\left(I_{\infty}\right)$ and to $\mathrm{M}\left(\mathrm{I}_{0}\right)$, and is the simplest choice for any of these four parametrizing matrices. Based upon such choices, a very simple design procedure which achieves the basic stabilization of the singularly perturbed system $\mathrm{P}(\mathrm{s}, \mathrm{e})$ of type (5) is formalizable as follows:

\section{DESIGN-PROCEDURE I}

Step 1: Use (12) and (13) to obtain the Doubly Coprime Factorizations, $\left(N_{s}, D_{s}, \bar{N}_{s}, D_{s}, X_{s}, Y_{s}, \bar{X}_{s}, \bar{Y}_{s}\right)$ and $\left(N_{f}, D_{f}, \bar{N}_{f}, \bar{D}_{f}, X_{f}, Y_{f}, \bar{X}_{f}, \bar{Y}_{f}\right)$ of the reduced-order models $P_{S}(s)$ and $P_{F}(p)$ corresponding to $P(s, e)$.

Step 2: Use the constructive procedure of the proof of Theorem 2 to obtain a dc-blocking $\mathrm{X}_{3}$, and obtain its corresponding Bezout element element $Y_{3}$.

Step 3: Form the controllers $C_{s}(s)=Y_{s}(s)^{-1} X_{s}(s)$ and $C_{F}(p)=Y_{3}(p)^{-1} X_{3}(p)$.

Step 4: Connect the component systems $\mathrm{P}(\mathrm{s}, \mathrm{e}), \mathrm{C}_{\mathrm{s}}(\mathrm{s})$ and $\mathrm{C}_{\mathrm{F}}(\mathrm{es})$ into the configuration:

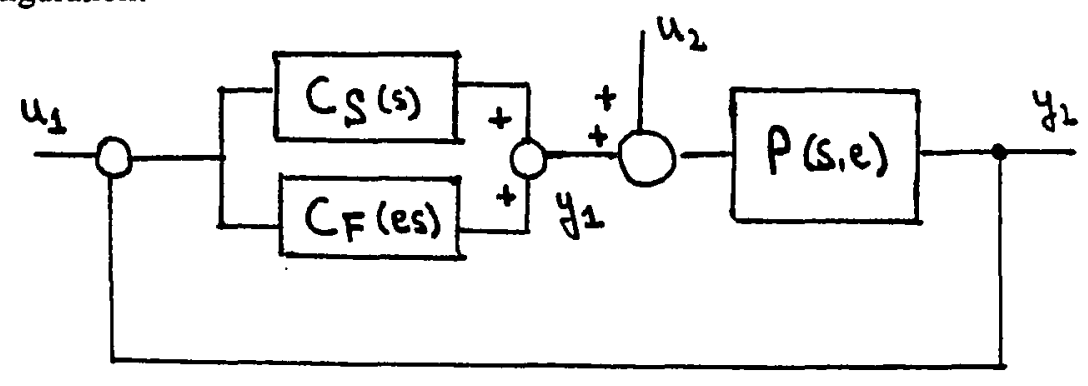

In the above basic feedback configuration, the transfer relationship between $\left(u_{1}^{t} u_{2}^{b}\right)^{t}$ and $\left(y_{1}^{t} y_{2}^{t}\right)^{t}$ will have been stabilized. 
For illustrating the present design procedure, and as well two further design procedures which are subsequently to be formalized, one particular system is chosen to run through the several examples. For the convenience of hand calculation, this system is low-dimensional, and it represents the scalar rather than the multivariable case. All steps in the procedures are both mathematically and conceptually extremely simple. Since the multiplication operation is commutative in the scalar case, the distinction between right- and left-parametrizations collapses, and the formulas (7) through (10), (12) through (17) and (26) through (29) are immediately simplified. For multivariable applications, the designs are presented specifically in right-handed form, with the R-dependent controllers. Corresponding design procedures involving the lefthanded Q-dependent controllers are, of course, likewise formulable.

\section{EXAMPLE 1:}

We introduce the singularly perturbed system:

$$
\begin{gathered}
{\left[\begin{array}{c}
\dot{x} \\
\dot{z}
\end{array}\right]=\left[\begin{array}{cc}
4 & 2 \\
1 / \mathrm{e} & 1 / \mathrm{e}
\end{array}\right]\left[\begin{array}{l}
\mathrm{x} \\
\mathrm{z}
\end{array}\right]+\left[\begin{array}{c}
1 \\
1 / \mathrm{e}
\end{array}\right] \mathrm{u} \equiv\left[\begin{array}{cc}
\mathrm{A}_{11} & \mathrm{~A}_{12} \\
\mathrm{~A}_{21} / \mathrm{e} & A_{22} / \mathrm{e}
\end{array}\right]\left[\begin{array}{l}
\mathrm{x} \\
\mathrm{z}
\end{array}\right]+\left[\begin{array}{c}
\mathrm{B}_{1} \\
\mathrm{~B}_{2} / \mathrm{e}
\end{array}\right] \mathrm{u}} \\
\mathrm{y}=\left[\begin{array}{l}
12
\end{array}\right]\left[\begin{array}{l}
\mathrm{x} \\
\mathrm{z}
\end{array}\right] \equiv\left[\begin{array}{ll}
\mathrm{C}_{1} & \mathrm{C}_{2}
\end{array}\right]\left[\begin{array}{l}
\mathrm{x} \\
\mathrm{z}
\end{array}\right]
\end{gathered}
$$

This system is both controllable and observable. Its exact transfer function can be computed as:

$$
P(s, e)=C(s I-A(e))^{-1} B(e)=\frac{(2+e) s-5}{e s^{2}-(4 e+1) s+2}
$$

The system's exact poles are computable from the quadratic formula:

$$
\mathrm{s}_{1,2}=2+\frac{1}{2 \mathrm{e}} \pm \frac{1}{2 \mathrm{e}} \sqrt{1+16 \mathrm{e}^{2}}
$$

Note that the system is unstable. One of the exact poles is slightly smaller than $s_{1}=2$, and the other is slightly larger than $s_{2}=(1 / e)+2$. The approximating reduced-order models, as calculated 
by Luse's methods, are:

$$
\begin{gathered}
P(s, 0)=\frac{2 s-5}{-s+2} \\
P\left(\frac{p}{e}, e\right]_{e=0}=\frac{(2+e) \frac{p}{e}-5}{e \frac{p^{2}}{e^{2}}-(1+4 e) \frac{p}{e}+2} l_{c=0}=\frac{2}{p-1}
\end{gathered}
$$

The approximate poles are thus located at $s=2$ and at $\mathrm{p}=\mathrm{es}=1$.

From the time domain viewpoint, since $A_{0} \equiv A_{11}-A_{12} A_{22}^{-1} A_{21}=2, B_{0} \equiv B_{1}-A_{12} A_{22}^{-1} B_{2}=-1$, $C_{0} \equiv C_{1}-C_{2} A_{22}^{-1} A_{21}=-1$ and $D_{0} \equiv-C_{2} A_{22}^{-1} B_{2}=-2$, the slow reduced-order subsystem is given by:

$$
\begin{aligned}
& \dot{x}=2 x-u \\
& y=-x-2
\end{aligned}
$$

and has the transfer function:

$$
P_{S}(s)=\frac{C_{0} B_{0}}{s-A_{0}}+D_{0}=\frac{(-1)(-1)}{s-2}-2=\frac{-2 s+5}{s-2}
$$

This unstable slow subsystem is both controllable and observable. Using $A_{22}=1, B_{2}=1$ and $C_{2}=2$, the fast reduced-order subsystem is given by:

$$
\begin{gathered}
\dot{z}=z+u \\
y=2 z
\end{gathered}
$$

and has the transfer function:

$$
\mathrm{P}_{\mathrm{F}}(\mathrm{p})=\frac{\mathrm{B}_{2} \mathrm{C}_{2}}{\mathrm{p}-\mathrm{A}_{22}}==\frac{2}{\mathrm{p}-1}
$$

The fast subsystem, which is also unstable, is as well also both controllable and observable. Since the normal rank of the given system is 2 and since each of the reduced-order subsystems has rank 1 , this system has no "lost" poles.

Lemma 1 is now used to obtain Doubly Coprime Factorizations for the slow and fast sub- 
systems. The convenient choice of $K_{s}=-3$ for the slow controller-gain yields $A_{0}-B_{0} K_{s}=-1$; the choice of $L_{s}=-3$ for the slow observer-gain yields $A_{0}-L_{s} C_{0}=-1$. The consequent Doubly Coprime Factorization for the slow subsystem is rendered by:

$$
\begin{array}{ll}
\mathrm{N}_{s}=\overline{\mathrm{N}}_{\mathrm{s}}=\frac{5-2 \mathrm{~s}}{\mathrm{~s}+1}=\mathrm{n}_{\mathrm{s}} & \mathrm{X}_{\mathrm{s}}=\overline{\mathrm{X}}_{\mathrm{s}}=\frac{9}{\mathrm{~s}+1}=\mathrm{x}_{\mathrm{s}} \\
\mathrm{D}_{\mathrm{s}}=\overline{\mathrm{D}}_{\mathrm{s}}=\frac{s-2}{\mathrm{~s}+1}=\mathrm{d}_{\mathrm{s}} & \mathrm{Y}_{\mathrm{s}}=\overline{\mathrm{Y}}_{\mathrm{s}}=\frac{\mathrm{s}+22}{\mathrm{~s}+1}=\mathrm{y}_{\mathrm{s}}
\end{array}
$$

The convenient choices of $K_{f}=2$ for the fast controller-gain, yielding $A_{22}-B_{2} K_{f}=-1$, and of $L_{f}=1$ for the fast observer-gain yielding $A_{22}-L_{1} C_{2}=-1$, renders the Doubly Factorization for the fast subsystem:

$$
\begin{array}{ll}
\mathrm{N}_{\mathrm{f}}=\overline{\mathrm{N}}_{\mathrm{f}}=\frac{2}{\mathrm{p}+1}=\mathrm{n}_{\mathrm{f}} & \mathrm{X}_{\mathrm{f}}=\overline{\mathrm{X}}_{\mathrm{f}}=\frac{2}{\mathrm{p}+2}=\mathrm{x}_{\mathrm{f}} \\
\mathrm{D}_{\mathrm{f}}=\overline{\mathrm{D}}_{\mathrm{f}}=\frac{\mathrm{p}-1}{\mathrm{p}+1}=\mathrm{d}_{\mathrm{f}} & \mathrm{Y}_{\mathrm{f}}=\overline{\mathrm{Y}}_{\mathrm{f}}=\frac{\mathrm{p}+3}{\mathrm{p}+1}=\mathrm{y}_{\mathrm{f}}
\end{array}
$$

Since $x_{s}$ is already strictly proper, one strictly proper stabilizing controller for $P_{S}(s)$ is given by:

$$
\mathrm{C}_{\mathrm{s}}(\mathrm{s})=\frac{\mathrm{x}_{\mathrm{s}}}{\mathrm{y}_{\mathrm{s}}}=\frac{9 /(\mathrm{s}+1)}{(\mathrm{s}+22) /(\mathrm{s}+1)}=\frac{9}{\mathrm{~s}+22}
$$

Consequently, the set of all strictly proper stabilizing controllers for the slow subsystem is given by:

$$
\left\{C \text { in } \operatorname{Stab}\left(P_{s}(s)\right): C(\infty)=0\right\}=\left\{\frac{x_{s}+R d_{s}}{y_{s}-R_{s}}\right\}=\left\{\frac{9+R(s-2)}{(s+22)-R(5-2 s)} ; R \text { in } S(s) \text { and strictly proper }\right\}
$$

$\mathrm{x}_{\mathrm{f}}$, obtained from the Lemma, does not possess the dc-blocking property. To obtain a dcblocking Bezout element related to the fast subsystem, the constructive procedure of the Proof of Theorem 1 must be used. We shall choose for $f$, that dc-blocking member of $S(p)$ which is to be 
coprime in $S(p)$ with $d_{f}$ :

$$
\mathrm{f}=\frac{\mathrm{p}}{\mathrm{p}+1}
$$

By elementary polynomial methods, it is possible to find elements $a_{1}$ and $b_{1}$ in $S(p)$ which satisfy the Bezout equation $a_{1} d_{f}+b_{1} f=1$, namely:

$$
a_{1} d_{f}+b_{1} f \equiv\left(\frac{p-1}{p+1}\right]\left(\frac{p-1}{p+1}\right)+\left[\frac{4}{p+1}\right]\left(\frac{p}{p+1}\right)=1
$$

Therefore, the elements $a$ and $b$ which satisfy the relation $a d_{f}+b f=x$ are:

$$
\mathrm{a}=\frac{2}{\mathrm{p}+1} \cdot \frac{\mathrm{p}-1}{\mathrm{p}+1} \quad \mathrm{~b}=\frac{2}{\mathrm{p}+1} \cdot \frac{4}{\mathrm{p}+1}
$$

By construction, $\mathrm{f}$ divides $\left(\mathrm{x}-\mathrm{ad}_{\mathrm{f}}\right)$.

It is next required to form $\hat{x}=x_{f}+r d_{f}$ and $\hat{y}=y_{F}-m_{f .}$ In the general Proof, the matrix $R$ is equal to the matrix $-A D^{\text {adj; }}$; but in the SISO case, $R$ is represented by the scalar $r, A$ is represented by the scalar a and the adjoint of the matrix, by default, is unity. Consequently, $r=-a$, and:

$$
\begin{gathered}
\hat{x} \equiv x_{f}+r d_{f}=\left(\frac{2}{p-1}\right]-\left[\frac{2(p-1)}{(p+1)^{2}}\right]\left[\frac{p-1}{p+1}\right]=\frac{8 p}{(p+1)^{3}} \\
\hat{y} \equiv y_{f}-m_{f}=\left[\frac{p+3}{p+1}\right]+\left(\frac{2(p-1)}{(p+1)^{2}}\right]\left[\frac{2}{p+1}\right]=\frac{p^{3}+5 p^{2}+11 p-1}{(p+1)^{3}}
\end{gathered}
$$

One dc-blocking stabilizing compensator for the fast subsystem, $P_{F}(p)$, is now known to be:

$$
C_{F}(p)=\frac{\hat{x}}{\hat{y}}=\frac{8 p}{p^{3}+5 p^{2}+11 p-1}
$$

Note that this stabilizing compensator is not itself stable! Also note that this compensator, a compensator for a first-order system, is itself third-order!

Finally, the set of all dc-blocking stabilizing compensators for $P_{F}(p)$ is given by:

$$
\left\{\operatorname{Cin} \operatorname{Stab}\left(P_{F}(p)\right): C(0)=0\right\}=\left\{\frac{\hat{x}+R d_{f}}{\hat{y}-R n_{f}}\right\}=\left\{\frac{8 p+R(p-1)(p+1)^{2}}{\left(p^{3}+5 p^{2}+11 p-1\right)-R\left(2(p+1)^{2}\right)} ; R \text { in } S(s), R(0)=0\right\}
$$


Steps 1 through 3 of the Design Procedure are now completed. The implementation of Step 4 is immediate, and leads to the stabilized system:

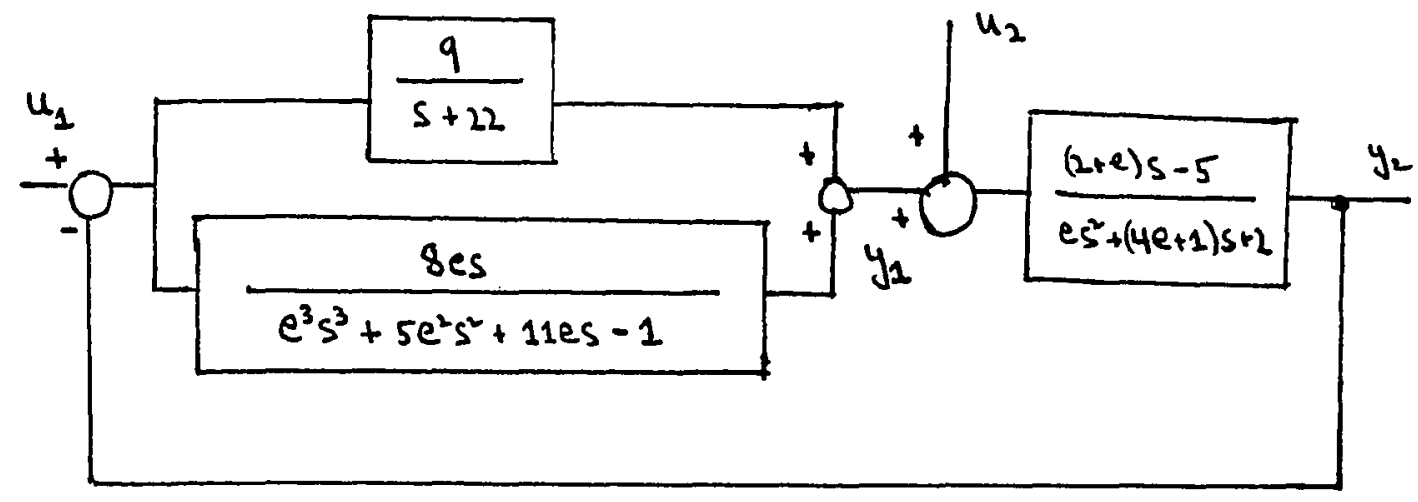

4.2.5 Design Procedure \#2: Pole Placement within a Specified Subregion of the Complex Plane

Usually, more than the mere stabilization of the plant is required. We next suppose that it is required to place the closed-loop poles in some specified subregion of the open left half plane, allowing this region to be shaped as follows:

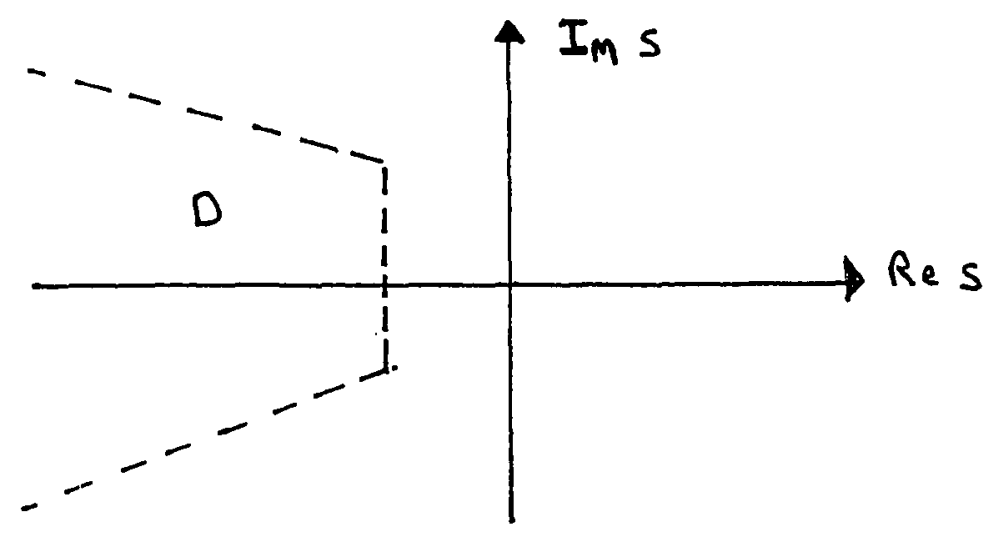

Figure 12 
Algebraically, the permitted regions are described by the expressions:

$$
\{D=\{s: \operatorname{Re} S<-a,|\operatorname{Im} s|<b|\operatorname{Re} s|\}: a, b \text { are positive Real numbers }\}
$$

The point at infinity is understood to be contained in the forbidden region, $D^{c}$, the complement of D in the Complex Plane. When mere stability was called for, the permittted region was the open left half plane, and the forbidden region was $\mathrm{C}_{\mathrm{e}}^{+}$, the closed extended right half plane. Now that a more stringent form of stability may be required, the forbidden region is enlarged and the permitted region is made more restrictive. By having its poles placed in the region $D$, the plant is meted to more specific design criteria than stability alone; in physical terms, placement of the poles in D guarantees some minimum damping and some maximum settling time on the responses of the feedback system.

To further formalize these matters, $S_{D}$ is defined as the Ring of proper rational transfer functions which have all their poles in the region D. The Ring S represents, of course, that special case in which the Region D is the open left half plane. Like $S, S_{D}$ is made into an Euclidean Domain with the degree function:

$$
\operatorname{deg}(): S_{D} \rightarrow Z^{+}: f \rightarrow \text { number of zeros of } f \text { in } D^{c}
$$

Again like $S, S_{D}$ is topologized in terms of the norm:

$$
|| f||=\sup _{\sin D^{*}}|f(s)|
$$

For fairly obvious technical reasons, D must be an open subset of the Complex Plane which is symmetric with respect to the Real axis. Then, modulo at most a few technical considerations, all the basic algebraic properties and all the basic topological properties of $S$ pertain also to $S_{\mathrm{D}}$. In particular, the arbitrary matrix in $M\left(R(s)\right.$ ) supports coprime factorizations over $M\left(S_{D}\right)$, and 
consequently for any plant $\mathrm{P}$ it is possible to obtain expressions for

$$
\operatorname{Stab}_{D}(P) \equiv\left\{C \text { in } M(R(s)): H(P, C) \text { in } M\left(S_{D}\right)\right\}
$$

in terms of free affine parameters which are matrices in $M\left(S_{D}\right)$. L. Pernebo [24-25], who builds a complete and extremely sophisticated algebraic theory of compensator design upon the properties of $S_{D}$, refers to $S_{D}$ as a Ring of "generalized polynomials". Algebraically, the elements of $S_{D}$ are akin to the polynomials, which, like them, form an Euclidean Domain, rather than to the rational functions, which are Field-structured.

The square matrix A over the Complex Field is defined as D-stable if all its eigenvalues lie in D. The pair $(A, B)$ pertaining to the State Space realization of a given system is defined as D-stabilizable if all the noncontrollable modes (i.e. all the input decoupling zeros) lie in D; and the pair $(\mathrm{A}, \mathrm{C})$ is defined as D-detectable if all the nonobservable modes (i.e all the output decoupling zeros) lie in $\mathrm{D}$. When the pair $(\mathrm{A}, \mathrm{B})$ is $\mathrm{D}$-stabilizable, there exists a Complex matrix $\mathrm{K}$ such that $(A-B K)$ is D-stable; likewise, if the pair $(A, C)$ is D-detectable, there exists a Complex matrix $\mathrm{L}$ such that (A-LC) is D-stable.

Lemma 1 can in this context be read as providing Doubly Coprime Factorizations over $S_{D}$ for plants whose slow and fast subsystems are both D-stabilizable and D-detectable. Quite simply, if, in the statement of Lemma $1,(A, B)$ and $(A, C)$ are required restrictively to be $D$ stabilizable and D-detectable, and if the matrices $\mathrm{K}$ and $\mathrm{L}$ are chosen to make respectively (ABK) and (A-LC) D-stable, then all eight matrices yielded by the Lemma -- N, D, $\bar{N}, \bar{D}, X, Y, \bar{X}$ and $\bar{Y}$-- remain specifically in $M\left(S_{D}\right)$. In this way, the Lemma adapts to the present purposes of pole placement within the specified subregion. A design procedure for achieving such poleplacement which is only slightly more complicated than Design Procedure I is thus formalizable as follows: 


\section{DESIGN-PROCEDURE II}

Step 1: Assuming that $\left(A_{0}, B_{0}\right)$ is D-stabilizable and $\left(A_{0}, C_{0}\right)$ is $D$ detectable, select $K_{s}$ to make $A_{0}-B_{0} K_{s} D$-stable and $L_{5}$ to make $A_{0}-L_{5} C_{0} D$ stable. Assuming that $\left(A_{22}, B_{2}\right)$ is D-stabilizable and $\left(A_{22}, C_{2}\right)$ is D-detectable, select $K_{f}$ to make $A_{22}-B_{2} K_{f} D$-stable and $L_{f}$ to make $A_{22}-L_{f} C_{2} D$-stable.

On these bases, obtain from (12) and (13) the Doubly Coprime Factorizations $\left(N_{s}, D_{s}, \bar{N}_{s}, \bar{D}_{s}, X_{s}, Y_{s}, \bar{X}_{s}, \bar{Y}_{s}\right)$ and $\left(N_{f}, D_{f}, \bar{N}_{f}, \bar{D}_{f}, X_{f}, Y_{f}, \bar{X}_{f}, \bar{Y}_{f}\right)$ for the reduced order models $\mathrm{P}_{\mathrm{S}}(\mathrm{s})$ and $\mathrm{P}_{\mathrm{F}}(\mathrm{p})$ corresponding to $\mathrm{P}(\mathrm{s}, \mathrm{e})$.

Step 2: Through the selection of the generating element $f$ which is coprime in $S_{D}$ with $\operatorname{det}\left(\bar{D}_{f}\right)$, follow the constructive procedure of the Proof of Theorem 2 to obtain a dc-blocking $X_{3}$ in $S_{D}$, and obtain its corresponding Bezout element $Y_{3}$.

Step 3: Form the controliers $C_{S}(s)=Y_{s}(s)^{-1} X_{s}(s)$ and $C_{F}(p)=Y_{3}(p)^{-1} X_{3}(p)$.

Step 4: Connect the component systems $P(s, e), C_{s}(s)$ and $C_{F}(e s)$ into the configuration:

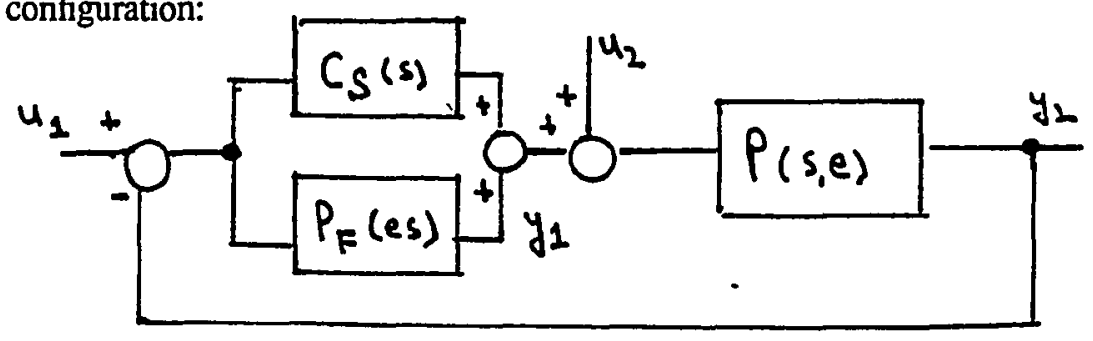

In the above basic feedback configuration, the poles of the transfer function between $\left(u_{1}^{\mathfrak{t}} u_{2}^{\mathfrak{t}}\right)^{\mathfrak{t}}$ and $\left(y_{1}^{\mathfrak{l}} y_{2}^{\mathfrak{t}}\right)^{\mathfrak{t}}$ will have been placed within the region $D$.

\section{Example 2:}

Continuing with the system of Example 1, we here suppose the permitted region to be described by:

$$
\mathrm{D}=\{\mathrm{s}: \operatorname{Re} \mathrm{s}<-2.5,|\operatorname{Im} \mathrm{s}|<|\operatorname{Re} \mathrm{s}|\}
$$

The convenient choices of $K_{3}=-5$ and $L_{3}=-5$ for the slow controller gain and the slow observer gain yield $A_{0}-B_{0} K_{s}=-3$ and $A_{0}-L_{s} C_{0}=-3$. The slow subsystem thus supports the Doubly Coprime 
Factorization:

$$
\begin{array}{ll}
N_{s}=\bar{N}_{s}=\frac{5-2 s}{s+3}=n_{s} & X_{s}=\bar{X}_{s}=\frac{25}{s+3}=x_{s} \\
D_{s}=\bar{D}_{s}=\frac{s-2}{s+3}=d_{s} & Y_{s}=\bar{Y}_{s}=\frac{s+58}{s+3}=y_{s}
\end{array}
$$

$x_{s}$, as anticipated, is strictly proper. Thus, one strictly proper D-stabilizing controller for $P_{S}(s)$ is given by:

$$
C_{s}(s)=\frac{x_{3}}{y_{s}}=\frac{25}{s+58}
$$

and the set of all strictly proper D-stabilizing controllers is given by:

$$
\left\{\mathrm{C} \text { in } \mathrm{Stab}_{\mathrm{D}}\left(\mathrm{P}_{\mathrm{S}}(\mathrm{s})\right): \mathrm{C}(\infty)=0\right\}=\left\{\frac{25+\mathrm{R}(\mathrm{s}-2)}{(\mathrm{s}+58)+\mathrm{R}(2 \mathrm{~s}-5)}: \mathrm{R} \text { in } \mathrm{S}_{\mathrm{D}}, \mathrm{R}(\infty)=0\right\}
$$

The convenient choices of $\mathrm{K}_{\mathrm{f}}=4$ for the fașt controller gain, yielding $A_{22}-B_{2} K_{F}=-3$ and of $L_{F}=2$ for the fast observer gain, yielding $A_{22}-L_{F} C_{2}=-3$, determine the following Doubly Coprime Factorization over $S_{D}$ of the fast subsystem:

$$
\begin{array}{ll}
N_{f}=\bar{N}_{f}=\frac{2}{p+3}=n_{f} & X_{f}+\bar{X}_{f}=\frac{8}{p+3}=x_{f} \\
D_{f}=\bar{D}_{f}=\frac{p-1}{p+3}=d_{f} & Y_{f}=\bar{Y}_{f}=\frac{8}{p+3}=y_{f}
\end{array}
$$

To obtain a $d c$-blocking Bezout element in $S_{D}$, we shall follow the constructive procedure of the Proof, choosing, for the generating dc-blocking member of $S_{D}(p)$ :

$$
f=\frac{p}{p+3}
$$


Elementary polynomial methods next find the elements:

$$
a_{1}=\frac{p-9}{p+3} \quad \text { and } \quad b_{1}=\frac{16}{p+3}
$$

to satisfy the Bezout equation, $a_{1} d_{r}+b_{1} f=1$. Therefore, the elements $a$ and $b$ which satisfy the relation $\mathrm{ad}_{\mathrm{f}}+\mathrm{bf}=\mathrm{x}$, and which thus enable $\mathrm{f}$ to be a divisor of $\mathrm{a}-\mathrm{d}_{\mathrm{f}}$, are:

$$
a=\frac{8}{p+3} \bullet \frac{p-9}{p+3} \quad b=\frac{8}{p+3} \bullet \frac{16}{p+3}
$$

Next to be computed are:

$$
\begin{gathered}
\hat{x}=x_{f}-a d_{f}=\frac{8}{p+3}-\frac{8(p-9)}{(p+3)^{2}} \bullet \frac{p-1}{p+3}=\frac{128 p}{(p+3)^{3}} \\
\hat{y}=y_{f}+a n_{f}=\frac{p+7}{p+3}+\frac{8(p-9)}{(p+3)^{2}} \bullet \frac{2}{p+3}=p^{3}+13 p^{2}+\frac{67 p-81}{(p+3)^{3}}
\end{gathered}
$$

One dc-blocking D-stabilizing controller for the fast subsystem $\mathrm{P}_{\mathrm{f}}(\mathrm{p})$ is thus known to be:

$$
C_{F}(p)=\frac{\hat{x}}{\hat{y}}=\frac{128 p}{p^{3}+13 p^{2}+67 p-81}
$$

and the set of all dc-blocking $D$-stabilizing controlers for $P_{F}(p)$ is given by:

$$
\left\{C \text { in } \operatorname{Stab}_{D}\left(P_{F}(p)\right): C(0)=0\right\}=\left\{\frac{128 p+R(p-1)(p+3)^{2}}{\left(p^{3}+13 p^{2}+67 p-81\right)-R\left(2(p+3)^{2}\right)}: R \text { in } S_{D}, R(0)=0\right\}
$$

Now the feedback system can be assimilated: 


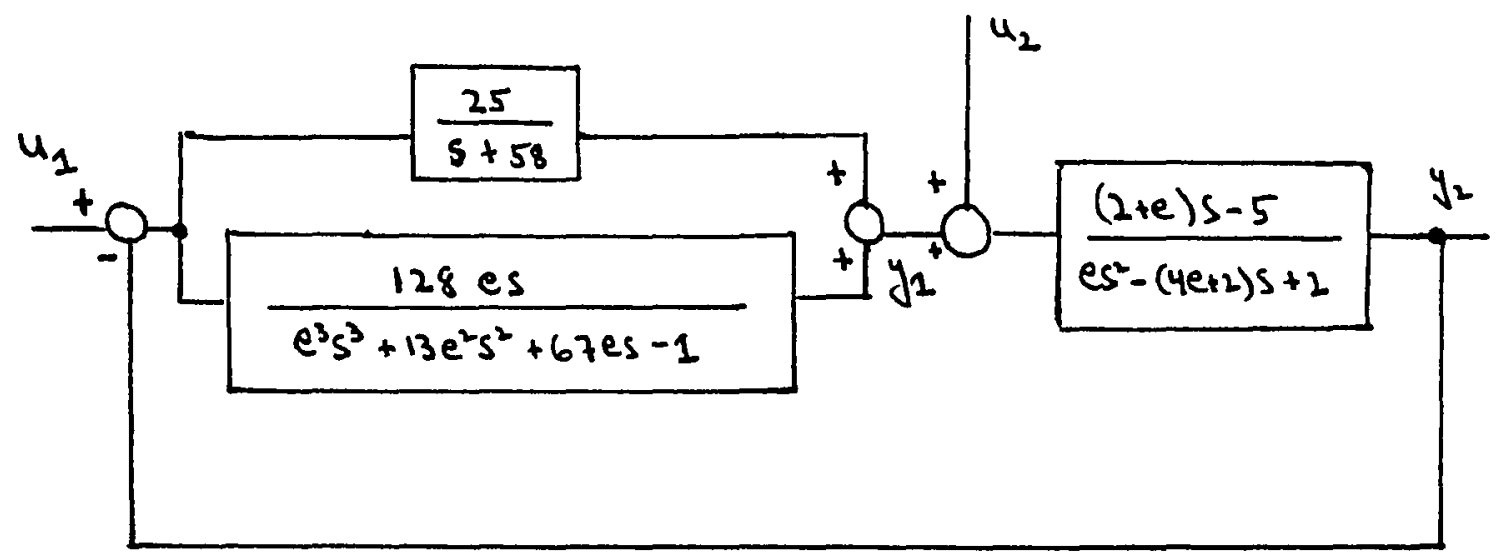

This feedback system is guaranteed to be overdamped, with none of its time-constants any longer than $(2.5)^{-1}$.

\subsubsection{Design Procedure \#3: Arbitrary Pole Placement by Algebraic Methods}

We next suppose that it is desired to further regulate $\mathrm{P}(\mathrm{s}, \mathrm{e})$, by placing both the slow poles and the fast poles at particular locations. In studying what pole-placement for the multivariable singularly perturbed system specifically entails, it behooves us to make use of the full algebraic formalism that is already at hand. The Transfer Matrices with which we have been working, generically denoted by $W(P, C)$, represent the transfer relationship between $\left(u_{1}^{t} u_{2}^{t}\right)^{t}$ and $\left(y_{1}^{t} y_{2}^{t}\right)^{t}$ in the basic configuration:

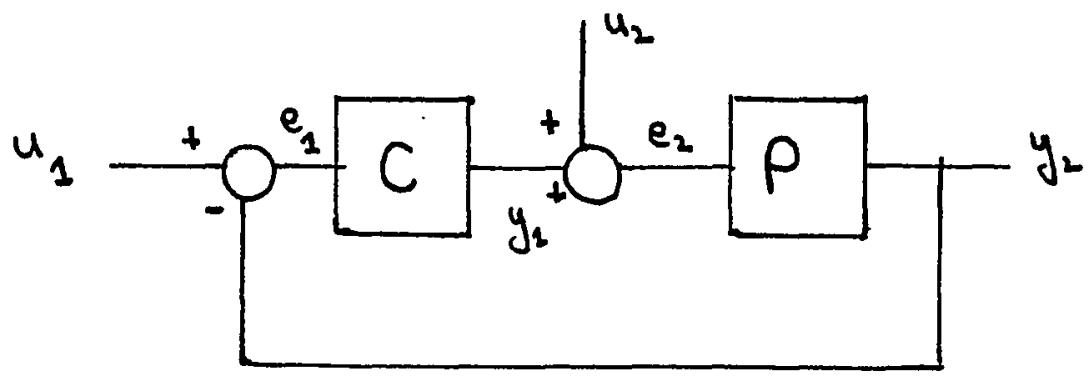

Figure 13 
The $(1,1),(1,2),(2,1)$ and $(2,2)$ submatrix entries of $W(P, C)$ thus describe, respectively, the four individual transfer relationships:

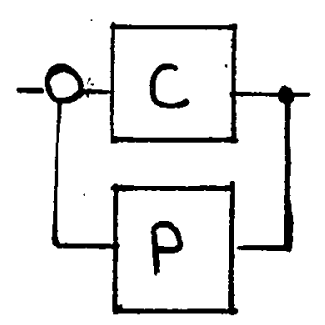

Figure 14a

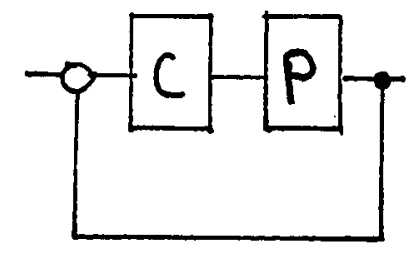

Figure 14b

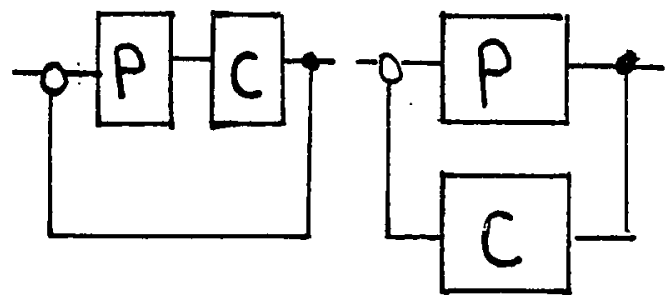

Figure 14c
Figure 14d

We may suppose now, for specificity, that a given matrix $R_{2}$ in $M\left(L_{\infty}\right)$ imparts desirable characteristics to the transfer matrix $\left(\mathrm{N}_{3} \mathrm{X}_{2}+\mathrm{N}_{3} \mathrm{R}_{2} \overline{\mathrm{D}}_{3}\right.$, the $(2,1)$ entry of $\mathrm{W}\left(\mathrm{P}_{\mathrm{S}} \mathrm{R}_{2}\right)$ in (28), and that likewise a given matrix $R_{3}$ in $M\left(I_{0}\right)$ imparts desirable characteristics to the transfer matrix $\left(N_{f} X_{3}+N_{f} R_{s} \bar{D}_{f}\right)$, the $(2,1)$ entry of $W\left(P_{F}, R_{3}\right)$. Then, compensators formed, in the light of (26) and (27), as $C_{S}(s)=\left(Y_{2}-R_{2} \bar{N}_{s}\right)^{-1}(s)\left(X_{2}+R_{2} D_{s}\right)(s)$ and $C_{F}(p)=\left(Y_{3}-R_{3} \bar{N}_{f}\right)^{-1}(p)\left(X_{3}+R_{3} D_{f}\right)(p)$ for the respective plants $P_{S}(s)$ and $P_{F}(p)$ will achieve these desirable transfer relationships in the configuration of Figure 14c, which corresponds to the $(2,1)$ entries. Once the parameters $R_{2}$ and $R_{3}$ have been identified, the determination of the compensators is immediate. The difficult matter is determination of the matrices $R_{2}$ and $R_{3}$ which realize agreed-upon "good" transfer relationships in given cases.

If $G_{s}(s)$ is a transfer matrix in $M\left(I_{\infty}\right)$ whose poles are desired as the slow poles of the closed-loop singularly perturbed system, and if $G_{f}(p)$ is a transfer matrix in $M\left(I_{0}\right)$ whose poles are e times the ones desired for its fast poles -- i.e. if $G_{s}(s)$ and $G_{f}(p)$ are the models that we wish the transfer murrices of the closed-loop slow and fast subsystems to match -- it is required to find the parametrizing strictly proper and dc-blocking matrices $R_{2}(s)$ and $R_{3}(p)$ which enable the closedloop system to match these models. In the general matrix case, it is not an entirely simple matter 
to solve for $\mathrm{R}$ in the equation:

$$
G(s)=N(s) X(s)+N(s) R(s) \bar{D}(s)
$$

In the first place, $\mathrm{N}$ is not necessarily square and may easily fail to be right-invertible. Secondly, and more seriously, $\overline{\mathrm{D}}^{-1}$, though guaranteed to exist in $M(R(s))$, is not guaranteed to belong to $\mathbf{M}(\mathbf{S})$-- yet $\mathrm{Q}$ is to reside in the specific subset $\mathrm{M}(\mathrm{I})$ of $\mathrm{M}(\mathrm{S})$ ! It is not at all clear that a straightforward algebraic solution for this $Q$ exists.

One recourse in this situation is to turn from Algebra to Functional Analysis, and to seek, by the suitable choice of $R$ in $M(I)$, to minimize the norm of the error-matrix, $E=G-(N X+N R \bar{D})$. An optimization problem having the structure:

$$
\min _{R \text { in } M(S)} J(R)=|| F-G R H \mid I_{\infty} \quad(F, G, H \text { in } M(S))
$$

is in fact a subject of attention in the current Control literature, under the heading of $\mathrm{H}_{\infty}-$ optimization or $\mathrm{H}_{\infty}$-Control Theory. The presently considered optimization problem would have the somewhat more restrictive form:

$$
\min _{\mathbf{R} \text { in } M(\mathbb{M})} J(R)=\|F-G R H\|_{\infty} \quad(G, H \text { in } M(S), F \text { in } M(I))
$$

These problems are actually quite complicated -- the pursuit of solutions is such an active current area of research precisely because most of the problems of this type are not yet satisfactorily solved.

Towards simplifying the above problem, and persisting in the quest for a purely algebraic solution, albeit a less straightforward one, we consider one further theorem from Control System Synthesis for adoption into a design procedure: 
Theorem 2 [32,p120]: Given plant $P$ in $M(R(s))$ and compensator $C$ in $M\left(R(s)\right.$ ) which stabilizes $P$. Let $P_{1}$ denote $P(I+C P)^{-1}$, the transfer matrix from $u$ to $y$ of the system:

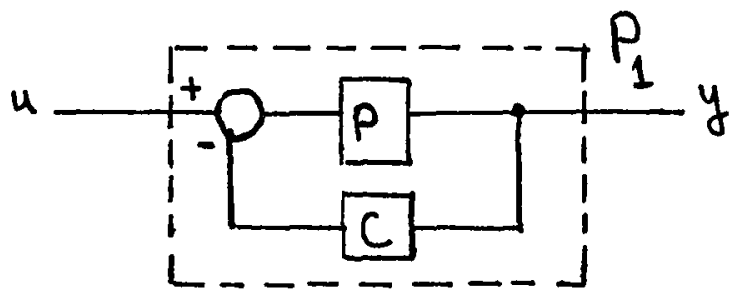

It follows that: $C+S t a b\left(P_{1}\right) \subseteq S t a b(P)$, with set-equality attaining if and only if $C$ belongs to $M(S)$.

Theorem 2 states that whenever the compensator $C_{1}$ stabilizes $P_{1}$, the parallel combination $\left(C_{1}+C\right)$ in fact stabilizes $P$. If $C$ is itself stable, then every stabilizing compensator for $P$ is in fact synthesizable in this manner. Consistently with our earlier conventions, $C_{1}$ must be considered as compensating $\mathrm{P}_{1}$, with specific reference to the following configuration:

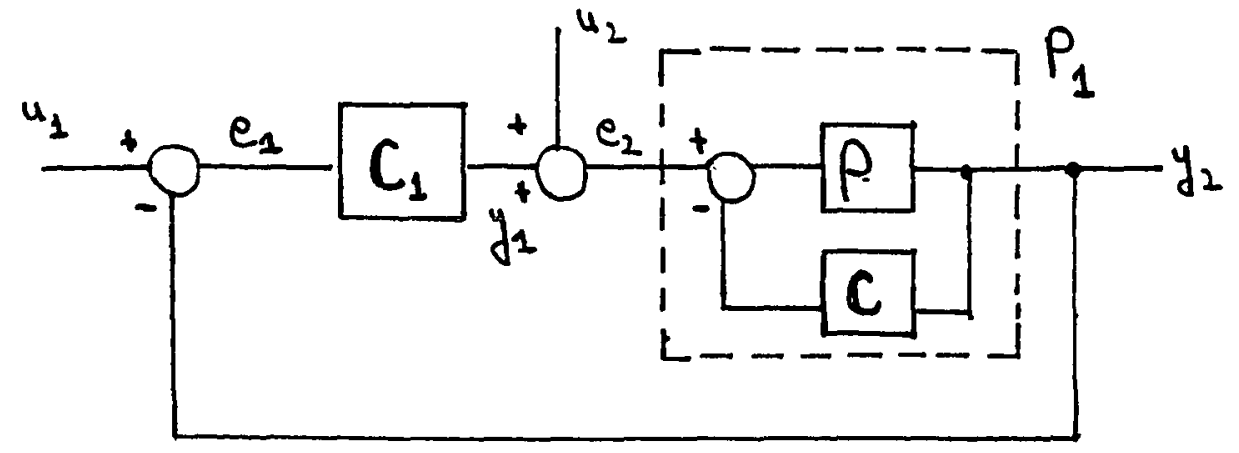

Figure 15

It is advantageous to work with the system $P_{1}$ rather than with the system $P$, because $P_{1}$, being already stable, supports the doubly coprime factorization, $\left(\mathrm{N}_{\mathrm{P}_{1}}, \mathrm{D}_{\mathrm{P}_{1}}\right)=\left(\mathrm{P}_{1}, \mathrm{I}_{1},\left(\overline{\mathrm{D}}_{\mathrm{P}_{1}}, \overline{\mathrm{N}}_{\mathrm{P}_{\mathrm{z}}}\right)=\left(\mathrm{I}_{2} \mathrm{P}_{1}\right)\right.$, $(X, Y)=(0, I),(\bar{Y}, \bar{X})=(I, 0)$. Consequently, $\operatorname{Stab}\left(P_{1}\right)$, the set of all controllers which preserve the stability of $P_{1}$ in the feedback configuration, is presentable in the shortened forms:

$$
\operatorname{Stab}\left(P_{1}\right)=\left\{\left(I-R P_{1}\right)^{-1} R: R \text { in } . I(S)\right\}=\left\{Q\left(I-P_{1} Q\right)^{-1}: Q \text { in } M(S)\right\}
$$


The Bezout elements $\mathrm{X}$ and $\overline{\mathrm{X}}$, being null matrices, possess both the dc-blocking property and the property of strict properness. Consequently, by (26) and (27):

$$
\begin{aligned}
& \left\{C_{1} \text { in Stab }\left(P_{1}\right): C_{1}(\infty)=0\right\}=\left\{\left(I-R_{2} P_{1}\right)^{-1} R_{2}: R_{2} \text { in } M\left(I_{\infty}\right)\right\}=\left\{Q_{2}\left(I-P_{1} Q_{2}\right)^{-1}: Q_{2} \text { in } M\left(I_{\infty}\right)\right\} \\
& \left\{C_{1} \text { in Stab }\left(P_{1}\right): C_{1}(0)=0\right\}=\left\{\left(I-R_{3} P_{1}\right)^{-1} R_{3}: R_{3} \text { in } M\left(I_{0}\right)\right\}=\left\{Q_{3}\left(I-P_{1} Q_{3}\right)^{-1}: Q_{3} \text { in } M\left(I_{0}\right)\right\}
\end{aligned}
$$

By (28) and (29), the closed-loop performance characteristics for the feedback system comprised of $P_{1}$ with the strictly proper compensator are given by:

$$
W\left(P_{1}, R_{2}\right)=\left[\begin{array}{cc}
R_{2} & -R_{2} P_{1} \\
P_{1} R_{2} & P_{1}\left(I-R_{2} P_{1}\right)
\end{array}\right] \quad \text { or by } \quad W\left(P_{1}, Q_{2}\right)=\left[\begin{array}{cc}
Q_{2} & Q_{2} P_{1} \\
-P_{1} Q_{2} & \left(I-P_{1} Q_{2}\right) P_{1}
\end{array}\right]
$$

Similarly, the closed-loop performance-characteristics for the feedback system comprised of $P_{1}$ with the dc-blocking compensator are given by:

$$
W\left(P_{1}, R_{3}\right)=\left[\begin{array}{cc}
R_{3} & -R_{3} P_{1} \\
P_{1} R_{3} & P_{1}\left(I-R_{3} P_{1}\right)
\end{array}\right] \quad \text { or by } \quad W\left(P_{1} Q_{3}\right)=\left[\begin{array}{cc}
Q_{3} & Q_{3} P_{1} \\
-P_{1} Q_{3} & \left(I-P_{1} Q_{3}\right) P_{1}
\end{array}\right]
$$

The matrices in (40) and (41) describe, specifically, the transfer relationships between $\left(u_{1}^{t} u_{2}^{t}\right)^{t}$ and $\left(y_{1}^{t} y_{2}^{t}\right)^{t}$ in the configuration of Figure 15 . Thanks to the stability of $P_{1}$, all but the $(2,2)$ entries in (40) and (41) possess particularly transparent shortened forms. In the $(1,1)$ spot, any strictly proper or dc-blocking transfer relationship can be immediately realized. The $(1,2)$ and $(2,1)$ entries may be filled by any strictly proper or dc-blocking left- or right- multiple of $P_{1}$. 
The general configuration of Figure 15 is shared by the following closed-loop systems, Figures $16 \mathrm{a}, 16 \mathrm{~b}$ and $16 \mathrm{c}$.

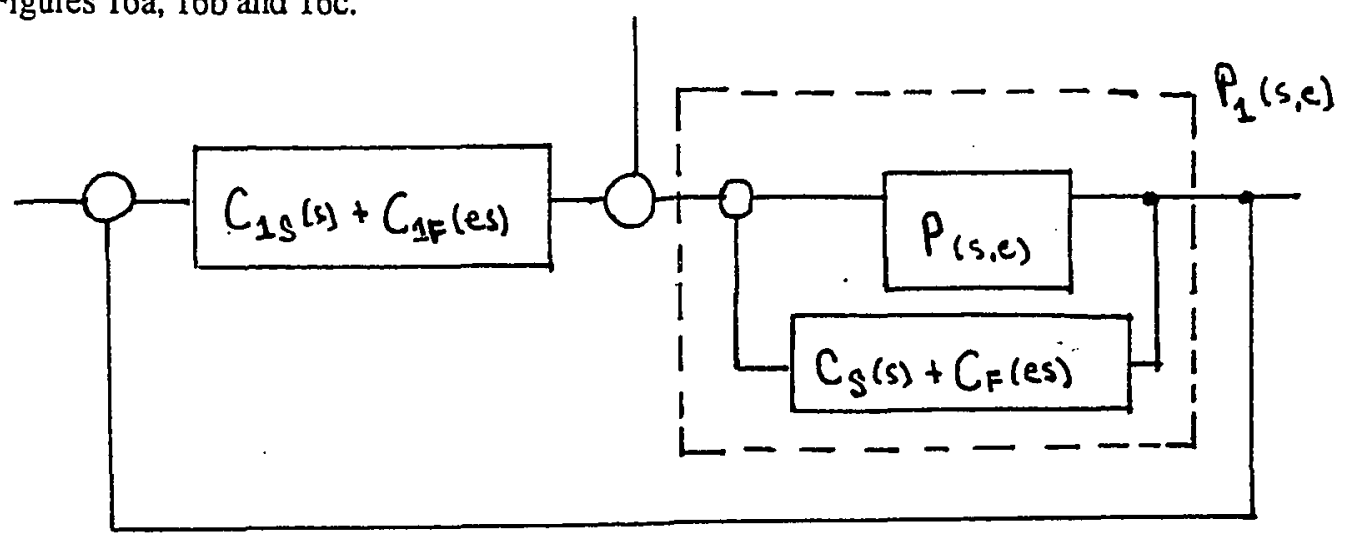

Figure 16a

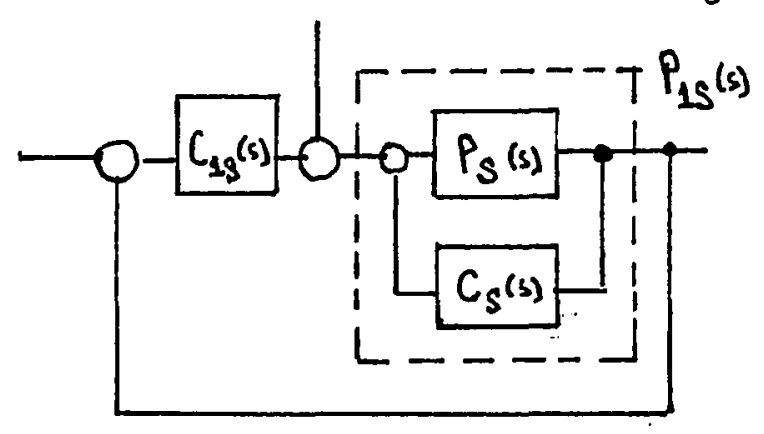

Figure 16b

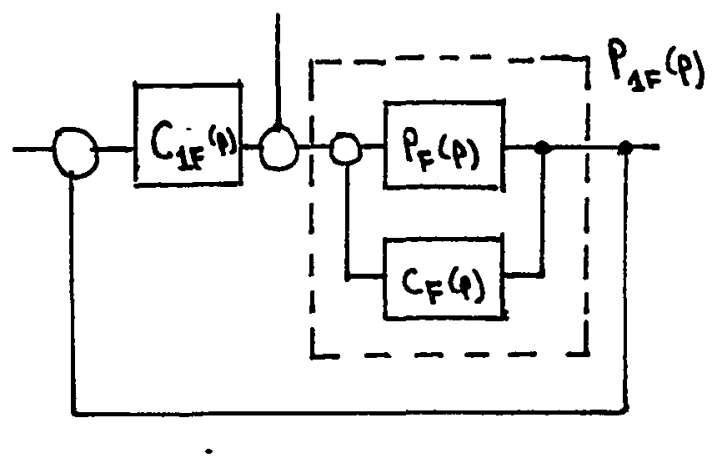

Figure 16c

In Figure $16, P_{S}(s)$ and $P_{F}(p)$ are understood, as usual, to be the slow and fast reduced-order approximating subsystems for the multiple-frequency-scale system, $\mathrm{P}(\mathrm{s}, \mathrm{e})$. With reference to this Figure, it is further taken that $C_{S}(\infty)=C_{F}(0)$ and that $C_{1 S}(\infty)=C_{1 F}(0)$. One final assumption is that $\left(I+C_{S}(\infty) P_{S}(\infty)\right),\left(I+C_{F}(\infty) P_{F}(\infty)\right),\left(I+C_{1 S}(\infty) P_{1 S}(\infty)\right)$ and $\left(I+C_{1 F}(\infty) P_{1 F}(\infty)\right)$ are all nonsingular as matrices over the Complex Field.

With Figure 16 in mind, a double application of Luse's Closed-Loop Theorem can be made. The Theorem is first applied only to the component subsystems, $P_{1}(s, e), P_{1 S}(s)$ and $P_{1 F}(p)$. This, the conventional application of the Theorem, determines $P_{1}(s, e)$ to be a two-frequency-scale system, whose $0(1)$ poles are the lost slow poles of $P(s, e)$ plus the poles of the closed-loop 
configuration $P_{1 S}(s)$, and whose $O(1 / \mathrm{e})$ poles are the lost fast poles of $P(s, e)$ plus $1 / \mathrm{e}$ times the poles of the closed-loop configuration $P_{1 F}(p)$. With this basic result in mind, the Theorem is secondly applied to the complete closed-loop configuration, comprised of the two-frequencyscale system $P_{1}(s, e)$ and the two-frequency-scale compensator $C_{1}(s, e)=C_{1 S}(s)+C_{1 F}(e s)$. In this second construction, the Theorem views the low-frequency and high-frequency reduced-order approximating models for Figure 16a as, precisely, Figures $16 \mathrm{~b}$ and 16c. By this application of the Theorem, the poles in Figure 15a are approximable by the open loop lost poles of $\mathrm{P}(\mathrm{s}, \mathrm{e})$ plus the closed-loop poles in Figure 16b plus 1/e times the closed-loop poles in Figure 16c.

In the design context, the interest is to place the poles of Figure 16a, through the placement of the poles in Figures $16 \mathrm{~b}$ and 16c. Theorem 2 here proves its usefulness, by enabling an effective two-stage procedure to be put to this effect. In the first stage of the procedure, any compensator which achieves the stabilization of $\mathrm{P}_{S}$ is chosen for the compensator $\mathrm{C}_{\mathrm{S}}$, and any compensator which stabilizes $P_{F}$ is chosen for $C_{F}$. At this stage, the simplest choices for $C_{S}$ and $C_{F}$ once again reflect the null choices for their relevant parametrizing matrices. The chosen $C_{S}$ and $C_{F}$ then incorporate into the descriptions of the stable plants $P_{1 S}$ and $P_{1 F}$. In the second stage of the procedure, the compensators $C_{1 S}$ and $C_{1 F}$ are determined. Since $P_{1 S}$ and $P_{1 F}$ are already stable, the compensators $C_{1 s}$ and $C_{1 F}$ are determined, respectively, according to (38) by either the right parameter $R_{2}$ or the left parameter $Q_{2}$ and according to (39) by either $R_{3}$ or $Q_{3}$. The relevant parameter is here selected, in the light of the performance characteristics (40) or (41), to meet the specific pole-placement criteria of a given case. At this point, the transparent forms of the entries in (40) and (41) translate into straightforward and flexible compensator design.

For example, if it is desired in the closed loop to achieve the strictly proper transfer matrix $G_{s}(s)$, we might simply choose $R_{2}(s)$ immediately as $G_{s}(s)$. Then, this desired transfer relationship is achieved between $u$ and $y$ in the following configuration: 


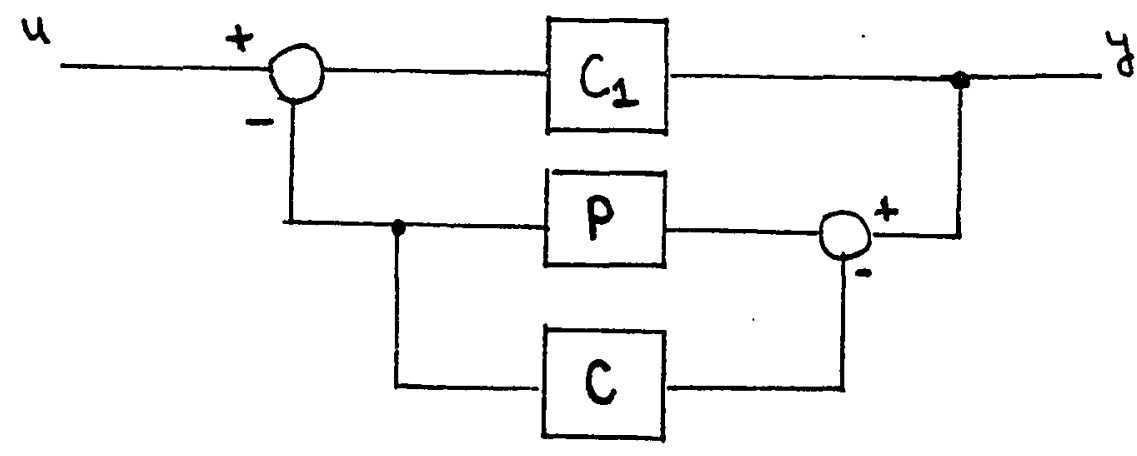

Figure 17

Figure 17 in effect re-draws Figure 15, highlighting the transfer relationship between the input $\mathrm{u}_{1}$ and the output $y_{1}$, which corresponds to the $(1,1)$ entry of the transfer matrix, $W\left(P_{1}, R_{2}\right)$. Admittedly, Figure 17, for placing the Compensator in the forward path and the Plant in the feedback path, is a nonstandard configuration, which cannot be recommended for implementation in most practical cases. Nonetheless -- for heuristic reasons -- we proceed to describe a formally valid pole-placing design strategy for the singularly perturbed system which utilizes this configuration. The putative object of the design procedure is to match the slow poles of the closed-loop system to the poles of the strictly proper model matrix $G_{3}(s)$ and to match the fast poles to $1 / \mathrm{e}$ times the poles of the dc-blocking model matrix $G_{f}(p)$.

\section{DESIGN-PROCEDURE IIa}

Step 1: Use (12) and (13) to obtain the Doubly Coprime Factorizations, $\left(\mathrm{N}_{s}, \mathrm{D}_{\mathrm{s}}, \overline{\mathrm{N}}_{\mathrm{s}}, \mathrm{D}_{\mathrm{s}}, \mathrm{X}_{\mathrm{s}}, \mathrm{Y}_{\mathrm{s}}, \overline{\mathrm{X}}_{\mathrm{s}}, \overline{\mathrm{Y}}_{\mathrm{s}}\right)$ and $\left(\mathrm{N}_{\mathrm{f}}, \mathrm{D}_{\mathrm{f}} \overline{\mathrm{N}}_{\mathrm{f}}, \overline{\mathrm{D}}_{\mathrm{f}}, \mathrm{X}_{\mathrm{f}}, \mathrm{Y}_{\mathrm{f}} \overline{\mathrm{X}}_{\mathrm{f}}, \overline{\mathrm{Y}}_{\mathrm{f}}\right)$, of the reduced-order models $P_{S}(s)$ and $P_{F}(p)$ corresponding to $P(s, e)$.

Step 2: Use the constructive procedure in the proof of Theorem 1 to obtain the dc-blocking Bezout element $X_{3}$, and obtain its corresponding Bezout element $Y_{3}$.

Step 3: Form the controllers $C_{s}(s)=Y_{s}^{-1}(s) X_{s}(s)$ and $C_{F}(p)=Y_{3}^{-1}(p) X_{3}(p)$.

Step 4: Let $P_{1 S}=P_{S}\left(I+C_{S} P_{S}\right)^{-1}$ and let $P_{1 F}=P_{F}\left(I+C_{F} P_{F}\right)^{-1}$. Then form the controllers $C_{1 S}=\left(I-G_{s} P_{1 S}\right)^{-1} G_{S}$ and $C_{1 F}=\left(I-G_{f} P_{I F}\right)^{-1} G_{F}$. 
Step 5: Connect the component systems $\mathrm{P}(\mathrm{s}, \mathrm{e}), \mathrm{C}_{\mathrm{S}}(\mathrm{s}), \mathrm{C}_{\mathrm{F}}(\mathrm{es}), \mathrm{C}_{1 \mathrm{~S}}(\mathrm{~s})$ and $\mathrm{C}_{\mathrm{IF}}(\mathrm{es})$ into the configuration:

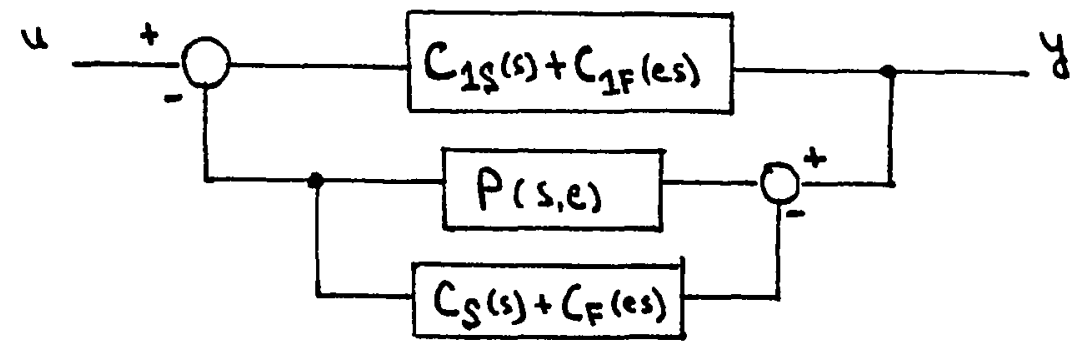

The slow poles of the transfer matrix between $u$ and $y$ in the above figure will have been placed approximately at the poles of $\mathrm{G}_{\mathrm{s}}(\mathrm{s})$ and its fast poles will have been placed at approximately $1 / \mathrm{e}$ times the poles of $G_{F}(p)$.

\section{EXAMPLE 3a:}

Continuing with the system of example 1 , we find steps 1 through 3 of the present design-procedure already completed. We shall suppose in the problem that $\mathrm{G}_{\mathrm{s}}(\mathrm{s})$ is stipulated by:

$$
G_{S}(S)=\frac{1}{(S+5)^{2}}
$$

and that $G_{f}(p)$ is stipulated by:

$$
\mathrm{G}_{\mathrm{p}}(\mathrm{p})=\frac{\mathrm{p}_{2}}{(\mathrm{P}+3)^{2}}
$$

In Step 4, the stabilized system $P_{15}(s)$ is computed as:

$$
\mathrm{P}_{1 \mathrm{~s}}(\mathrm{~s})=\frac{\frac{2 s-5}{-s+2}}{1+\frac{2 s-5}{-s+2} \cdot \frac{9}{s+22}}=\frac{-(2 s-5)(s+22)}{(s+1)^{2}}
$$

It is to be compensated with the pole-placing controller, $\mathrm{C}_{1 \mathrm{~s}}(\mathrm{~s})$, determined by:

$$
C_{1 S}(s)=\frac{\frac{1}{(s+5)^{2}}}{1+\frac{(2 s-5)(s+22)}{(s+5)^{2}(s+1)^{2}}}=\frac{(s+1)^{2}}{s^{4}+12 s^{3}+48 s^{2}+99 s-85}
$$


Similarly, $\mathrm{P}_{1 \mathrm{~F}}(\mathrm{p})$ is computed as:

$$
P_{1 F}(p)=\frac{\frac{2}{p-1}}{1+\frac{2}{p-1} \cdot \frac{8 p}{p^{3}+5 p^{2}+11 p-1}}=\frac{2\left(p^{3}+5 p^{2}+11 p-1\right)}{(p+1)^{4}}
$$

and then, $C_{1 F}(p)$ is determined by:

$$
C_{1 F}(p)=\frac{\frac{p^{2}}{(p+3)^{2}}}{1-\frac{p^{2}}{(p+3)^{2}} \cdot \frac{2\left(p^{3}+5 p^{2}+11 p-1\right)}{(p+1)^{4}}}=\frac{p^{2}(p+1)^{4}}{p^{6}+8 p^{5}+29 p}
$$

Note that the poles of $\mathrm{P}_{1 \mathrm{~S}}$ are cancelled by the zeros of $\mathrm{C}_{1 \mathrm{~S}}$, and that the poles of $\mathrm{P}_{1 \mathrm{~F}}$ are cancelled by the nonzero zeros of $\mathrm{C}_{\mathrm{iF}}$ !

In Step 5, the overall feedback system is assembled as follows:

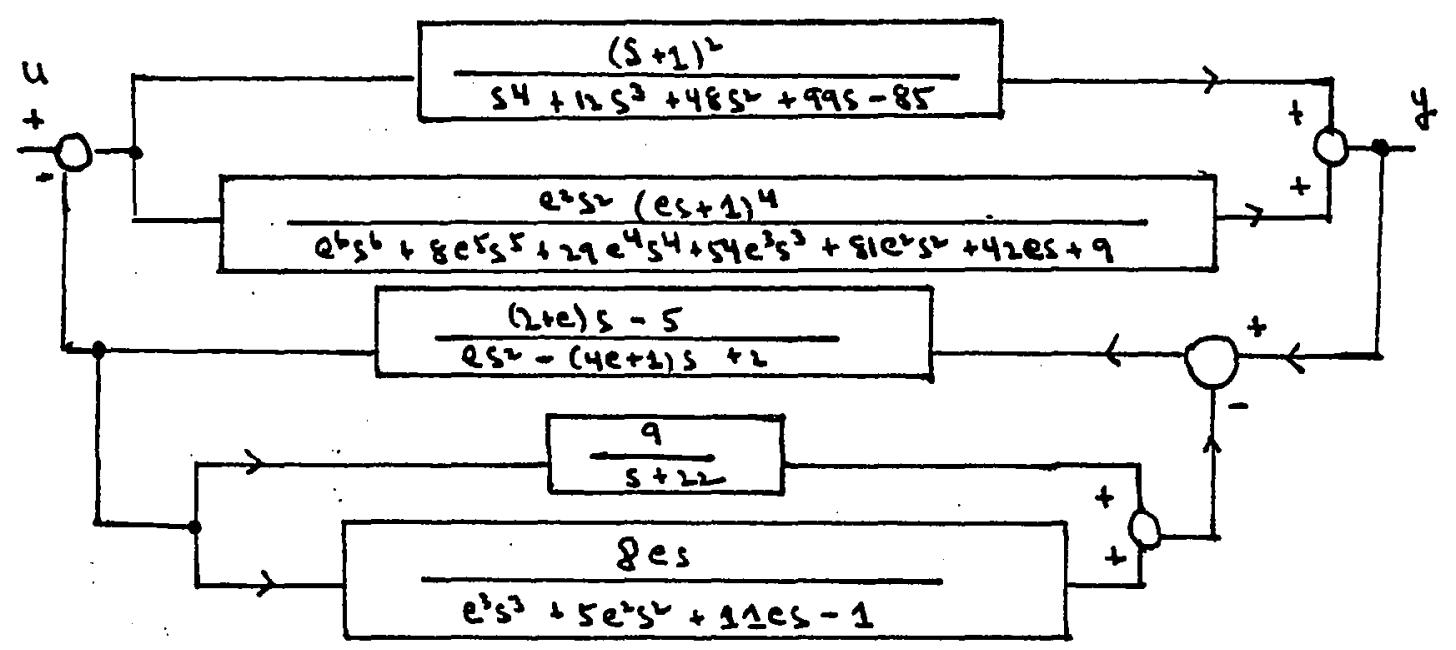

In the above configuration, the fourth-order transfer-function between $u$ and $y$ has a double pole at approximately $s=-5$ and another double pole approximately at $s=-3 / e$, thus achieving the goal of the design. For bounded inputs, all internal system signals are as well guaranteed to remain bounded. 
The configuration of Figure 15, with $u_{1}$ regarded as the input and $y_{2}$ as the output, unlike the configuration of Figure 17 (where $y_{1}$ is regarded as output) is generally suitable for practical designs. Working with the $(2,1)$ entries rather than with the $(1,1)$ entries of $(40)$ and $(41)$, any right multiple of $P_{1}$ can be achieved as the transfer matrix between $u_{1}$ and $y_{2}$ in Figure 15. The right-multiple of $P_{1}$, however, generally inherits the poles of $P_{1}$, and it is here desired to achieve any specified pole-placement for the singularly perturbed feedback system, not necesarily inclusive of the poles of $P_{1}$. It is significant that in Example 3 the poles of $P_{1 s}$ were cancelled by zeros of $C_{1 S}$, as were poles of $P_{1 F}$ by zeros of $C_{1 F}$, for such cancellations are intrinsic to the design procedure which achieves arbitrary exact pole-placement through purely algebraic means. These cancellations remained implicit in the formulation of Design Procedure IIIa. In the forthcoming design procedure, IIIb, cancellations will be explicitly called for. While not being disadvantaged vis a vis Design Procedure IIIa on the score of the cancellations, the following procedure has the serious advantage over its predecessor of utilizing a truly practical design configuration rather than one which enjoys merely formal correctness.

We here temporarily restrict attention to the case in which the plant $P$ is square and the transfer matrix of the stable compensated plant $P_{1}$ has full rank as a matrix over $R(s)$. In this case, if we choose the matrix $P_{1}^{2 d j} L$ for the parameter $R$, the transfer matrix between $u_{1}$ and $y_{2}$ will be, in accordance with $(40)$ or $(41), \operatorname{det}\left(\mathrm{P}_{1}\right) \mathrm{L}$. The parameter $\mathrm{R}=\mathrm{P}_{1}^{\mathrm{adj}} \mathrm{L}$ immediately determines the compensator $C_{1}=\left(I-P_{1}^{a d j} L P_{1}\right)^{-1} P_{1}^{2 d j} L$, in accordance with (38) or (39). Next, choosing $L$ as a diagonal matrix over $R(s)$ whose numerator polynomials cancel the (stabilized) poles of $P_{1}$ and whose denominator polynomials represent the poles of the desired transfer matrix $G_{s}(s)$ renders for the zeros of the transfer matrix between $u_{1}$ and $y_{2}$ in Figure 15 the zeros of $P_{1}$, and for its poles the poles of $\mathrm{G}_{s}$. A further achievement of this construction is that the transfer matrix between $u_{1}$ and $y_{1}$ has decoupled form. 
Given a square singularly perturbed system $P(s, e)$ of type (5) and the requirement to achieve, through separate-and-composite dynamic output controllers in the configuration of Figure 15 , the approximate placement of its slow and fast poles according to the poles of the strictly proper transfer matrix $G_{s}$ and $1 / e$ times the poles of the dc-blocking transfer matrix $G_{f}$, a twcstage purely algebraic procedure can be formalized as follows:

\section{DESIGN-PROCEDURE Mb}

Step 1: Use (12) and (13) to obtain the Doubly Coprime Factorizations, $\left(N_{s}, D_{s}, \bar{N}_{s}, D_{s}, X_{s}, Y_{s}, \bar{X}_{s}, \bar{Y}_{s}\right)$ and $\left(N_{f}, D_{f}, \bar{N}_{f}, \bar{D}_{f}, X_{f}, Y_{f}, \bar{X}_{f}, \bar{Y}_{f}\right)$, of the reduced-order models $\mathrm{P}_{\mathrm{S}}(\mathrm{s})$ and $P_{f}(\mathrm{p})$ corresponding to $\mathrm{P}(\mathrm{s}, \mathrm{e})$.

Step 2: Use the constructive procedure in the proof of Theorem 1 to obtain the dc-blocking Bezout element $X_{3}$, and obtain its corresponding element $Y_{3}$.

Step 3: Form the controllers $C_{S}(s)=Y_{s}^{-1}(s) X_{3}(s)$ and $C_{F}(p)=Y_{3}^{-1}(p) X_{3}(p)$.

Step 4: Form $P_{1 S}=P_{S}\left(I+C_{S} P_{S}\right)^{-1}$ and $P_{1 F}=P_{F}\left(I+C_{F} P_{F}\right)^{-1}$. Compute the adjoint matrices $P_{1 S}^{2 d j}(s)$ and $P_{1 F}^{2 d j}(p)$.

Step 5: Choose $L_{3}$, a strictly proper diagonal rational matrix whose numerators have zeros at the zeros of $P_{1 s}(s)$ and whose denominators have zeros at the poles of $\mathrm{G}_{\mathrm{s}}(\mathrm{s})$. Choose $\mathrm{L}_{\mathfrak{f}}$, a dc-blocking diagonal rational matrix with numerators having zeros at the zeros of $P_{1 F}(p)$ and denominators having zeros at the poles of $G_{f}(p)$. Let $R_{s}(s)=P_{1 s}^{a d j}(s) L_{s}(s)$ and let $R_{f}(p)=P_{1 F}^{2 d j}(p) L_{f}(p)$.

Step 6: Form the controllers $C_{1 S}=\left(I-R_{g} P_{1 S}\right)^{-1} R_{s}$ and $C_{l F}=\left(I-R_{g} P_{1 F}\right)^{-1} R_{f}$.

Step 7: Connect the component systems $P(s, e), C_{S}(s), C_{F}(e s), C_{1 S}(s)$ and $C_{1 F}(e s)$ into the configuration:

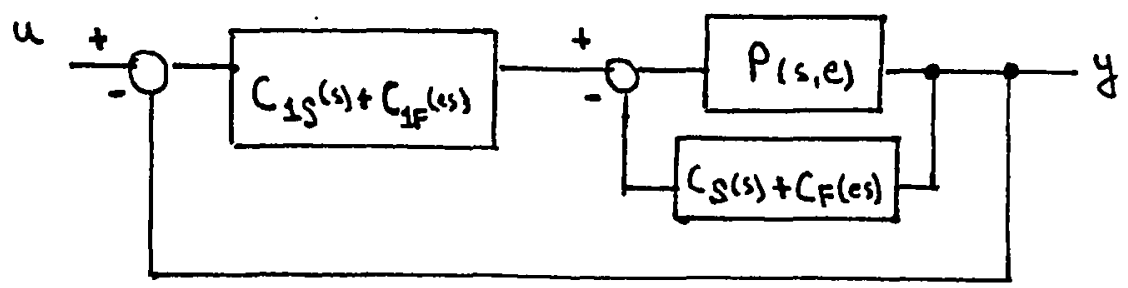


The poles of the transfer function from $u$ to $y$ are, approximately, the poles of $G_{s}(s)$ and $1 / \mathrm{e}$ times the poles of $\mathrm{G}_{\mathrm{f}}(\mathrm{p})$. Moreover, for bounded inputs, all internal system signals are guaranteed as well to remain bounded.

\section{EXAMPLE 3b:}

Continuing again with the system of the previous examples, we find steps 1 through 4 of the present design-procedure already completed. Recall that for computations in the scalar case all the adjoints are taken as unity.

We shall suppose in the problem that $G_{s}(s)$ is stipulated by:

$$
G_{s}(s)=\frac{1}{(s+5)^{3}}
$$

Then in Step 5, $\mathrm{L}_{5}(\mathrm{~s})$ can be chosen as:

$$
L_{s}(s)=\frac{(s+1)^{2}}{(s+5)^{3}}
$$

In Step 6, $C_{1 S(s)}$ is determined to be:

$$
C_{1 S}(s)=\frac{\frac{(s+1)^{2}}{(s+5)^{2}}}{1+\frac{(s+1)^{2}}{(s+5)^{2}} \cdot \frac{(2 s-5)(s+2)}{(s+1)^{2}}}=\frac{(s+1)^{2}}{s^{3}+17 s^{2}+114 s+15}
$$

We further suppose in the problem that $\mathrm{G}_{\mathrm{f}}(\mathrm{p})$ is stipulated by:

$$
G_{f}(p)=\frac{p^{2}}{(p+3)^{5}}
$$

Then in Step 5, $\mathrm{L}_{\mathrm{f}}(\mathrm{p})$ can be chosen as:

$$
L_{F}(p)=\frac{p(p+1)^{4}}{(p+3)^{5}}
$$

In Step $6 C_{1 F}(p)$ is then determined to be: 


$$
C_{1 F}(p)=\frac{\frac{p(p+1)^{4}}{(p+3)^{5}}}{1-\frac{p(p+1)^{4}}{(p+3)^{5}} \cdot \frac{2\left(p^{3}+5 p^{2}+11 p-1\right)}{(p+1)^{4}}}=\frac{p(p+1)^{4}}{p_{5}+15 p^{4}+104 p^{3}+356 p^{2}+623 p+405}
$$

In Step 7, the feedback system is finally assembled:

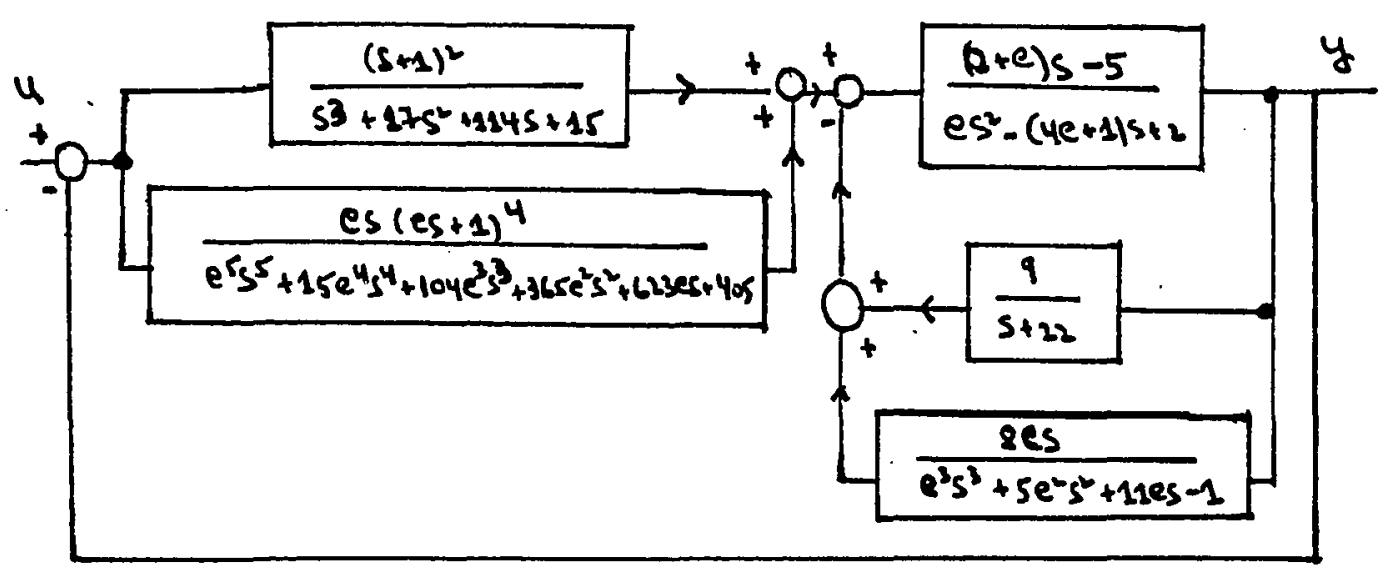

In the above configuration, the eighth-order transfer-function between $u$ and $y$ will have a triple pole at approximately $s=-5$ and a quintuple pole at approximately $s=-3 / e$, as required for the design.

\subsection{APPLICABILITY TO THE GENERAL MULTIPLE-TIME-SCALE SYSTEM}

Applicability of the present design procedures to given two-frequency-scale systems has required three basic conditions: (i) the systems may themselves have no unstable lost modes; (ii) both their slow and their fast reduced-order approximating models must be stabilizable and detectable; and (iii) Doubly Coprime Factorizations for each of these reduced-order subsystems must be attainable. 
For the system which is presented in explicit singularly perturbed form, Lemma I very conveniently provides explicit formulas for the Doubly Coprime Factorizations upon whose basis all the computations in the designs proceed. Doubly Coprime Factorizations for the lower-order approximating models which correspond to the general linear autonomous two-frequency-scale implicitly singularly perturbed system cannot likewise be displayed in a single paradigmatic form which immediately arraigns them for a sequence of computations. Still, these Doubly Coprime Factorizations are always obtainable on a case-by-case basis. The present design procedures are therefore indeed also applicable to the implicit singularly perturbed system, provided that the system itself has no unstable hidden modes and that its reduced order models are both stabilizable and detectable.

Rosenbrock, in his original work [27], has devised computational techniques for obtaining the coprime factorizations over polynomial matrices of the arbitrary proper rational transfer matrix. Vidyasagar has subsequently adapted many of Rosenbrock's techniques into procedures for attaining the coprime factorizations of the arbitrary rational matrix over stable proper matrices, i.e. over the Ring M(s). In Control System Synthesis [32], constructive algorithms necessarily accompany all the existence proofs -- including, of course, those proofs which pertain to the Doubly Coprime Factorizations of $M(R(s))$ elements over $M(S)$. These proofs and their associated algorithms are underlain and structured by those canonical forms -- the diagonal Smith and McMillan Forms and the triangular Hermite Forms -- which reveal the deep algebraic structures of the rational and associated polynomial and generalized-polynomial matrices. Consequently, given the ability to convert a given matrix into the appropriate canonical form, all the computations necessary for the attainment of those Doubly Coprime Factorizations over $M(S)$ which are requisite to the stabilization of the implicit singularly perturbed system are performable. 
One sequence of computations which obtains these Doubly Coprime Factorizations proceeds, in order, as follows:

(1) First, by Luse's methods, obtain $P_{S}(s)$ and $P_{F}(p)$ from $P(s, e)$, which represents the implicit singularly perturbed system.

(2) Second, produce arbitrary (i.e. not necessarily coprime) right and left matrix factorizations over $M(s)$ of $P_{S}(s)$ and $P_{F}(p)$-- namely, $\left(N_{1 s}, D_{1 s}\right),\left(\bar{D}_{1 s}, \bar{N}_{1 s}\right),\left(N_{1 f}, D_{1 f}\right)$, and $\left(\bar{D}_{1 f}, \bar{N}_{1 f}\right)$.

(3) Third, find a GCD (right or left, as appropriate) of the numerator matrix and denominator matrix in each of the four matrix fractions obtained in step (2).

(4) Fourth, "reduce" the four matrix fractions obtained in Step (2) by "extracting" the GCD's obtained in Step (3). These extractions yield coprime matrix factorizations, right and left, of $P_{S}(s)$ and $P_{F}(p)$-- namely, $\left(N_{s}, D_{s}\right),\left(\bar{D}_{s}, \bar{N}_{s}\right),\left(\mathbb{N}_{f}, D_{f}\right)$, and $\left(\bar{D}_{f}, \bar{N}_{f}\right)$.

(5) Fifth, find a pair of right-Bezout elements, $\left(X_{s}, Y_{3}\right)$, corresponding to $\left(N_{s}, D_{s}\right)$ and also the right-Bezout pair $\left(\mathrm{X}_{\mathrm{f}}, \mathrm{Y}_{\mathrm{f}}\right)$ corresponding to $\left(\mathrm{N}_{\mathrm{f}}, \mathrm{D}_{\mathrm{f}}\right)$.

(6) Sixth, for $P_{S}(s)$ and $P_{F}(p)$ in tum, from the RCF and the LCF obtained in Step 4, and the pair of right-Bezout elements obtained in Step 5, obtain the corresponding pair of leftBezout elements, $\left(\overline{\mathrm{X}}_{\mathrm{s}}, \overline{\mathrm{Y}}_{\mathrm{s}}\right)$ and $\left(\overline{\mathrm{X}}_{\mathrm{f}}, \overline{\mathrm{Y}}_{\mathrm{f}}\right)$, to comprise the Doubly Coprime Factorizations.

The interested reader will find the computational aspects of steps (2) through (7) fully explicated in Chapter Four of Control System Synthesis. The computational aspects of these and other related procedures are as well an active subject of independent interest in the current literature on Control.

A different route might also be taken to the attainment of the Doubly Coprime Factorizations associated with the implicit singularly perturbed system. Through multivariable Realization Theory [2,ch 6;10,ch 6], jointly controllable and observable state-space realizations of the $\mathrm{P}_{\mathrm{S}}(\mathrm{s})$ and $\mathrm{P}_{\mathrm{F}}(\mathrm{p})$ which correspond to the given $\mathrm{P}(\mathrm{s}, \mathrm{e})$ can be described. The Lemma can then yield 
Doubly Coprime Factorizations, based on these minimal state-space realizations. Since by hypothesis the subsystems are stabilizable and detectable, these Doubly Coprime Factorizations contain all the vital information pertaining to their stabilization. Upon the attainment of these Doubly Coprime Factorizations, the design proceeds exactly as for the explicit singulariy perturbed system. Through the one route or the other, the necessity of obtaining the Doubly Coprime Factorizations on a case-by-case basis represents an extra computational burden. But the present design procedures are otherwise applicable without disadvantage to the multipletime-scale system which is implicitly singularly perturbed. 


\section{CHAPTER V}

\section{CONCLUSIONS}

\subsection{EVALUATION OF THE DISSERTATION'S DESIGN PROCEDURES}

The perturbation parameter does not enter into any of the computations connected with the determination of the reduced-order controllers in the present design procedures. Mathematically, the fast controllers, as functions of the fast frequency variable $\mathrm{p}$, are e-independent quantities, as are the slow controllers in the frequency variable s. The perturbation parameter enters into the picture only when the composite controller, $C(s, e)=C_{s}(s)+C_{F}(p)$, comes to be formed. The latter is made parameter-dependent only because the fast frequency variable is scaled according to the relation $\mathrm{p}=\mathrm{es}$. Significantly, nowhere in the procedures have parameter-dependent factorizations been required. The parameter enters only in the most transparent and tractable way. Accordingly, those advantages which are usually sought in designs for the multiple-timescale systems connected with the avoidance of numerical ill-conditionedness, and as well those advantages connected with the reduction of dynamic order, are sustained by the present designs.

When an actual compensator is to be formed according to these design procedures, a specific value of the parameter must be chosen for it. Theoretically, the value $e_{1}$, which is used to form the compensator $C\left(s, e_{1}\right)$, matches the precise description of the plant's actual high-frequency dynamics. Strictly speaking, the compensator $C\left(s, e_{1}\right)$ is designed to regulate the specific member, 
$\mathrm{P}\left(\mathrm{s}, \mathrm{e}_{\mathrm{f}}\right)$, of the family of plants, $(\mathrm{P}(\mathrm{s}, \mathrm{e}))$. The fact that the designer's knowledge of the value of the perturbation parameter, meanwhile, is never exact yet poses no real practical difficulty, since the plant perturbations within a neighborhood of $e_{1}$ are only regular perturbations, and very ordinary continuity conditions on the descriptions of the plant and the compensator sufficiently guarantee robustness within a reasonable range of the expectable parameter uncertainty. $\mathrm{Bt}$ this same token, though, the present design procedures are suitable only to applications in which the plant's highfrequency dynamics can be reasonably well modelled.

The present design procedures suffer a number of patent liabilities. Firstly, these procedures do not minimize the dynamic order of the compensators. On the contrary, they tend to favor compensators of relatively high dynamic orders. In these designs, a compensator of order $n$ for the fast subsystem is in general written with coefficients up to the order $e^{n}$. Care must be taken to ensure that such extremely small coefficients are realistically implementable in the chosen hardware and software, lest these compensators be rendered ineffective through oversensitivity. The proclivity towards high-order seems, moreover, to be intrinsic to the designs, as it is is clearly foreshadowed by the various parametrized expressions for the stabilizing compensators.

Secondly, the procedures do not vouchsafe the stability of the stabilizing compensators themselves. Indeed, an unstable plant can formally be stabilized by an unstable controller, just as a stable plant can be destabilized by a controller which is itself stable. Still, it is generallly inadvisable to employ unstable components in practical applications. Beyond the results which have been utilized in the dissertation, Vidyasagar developes algebraic criteria for the "Strong Stabilizability" (i.e. the stabilizability by compensators which are themselves stable) of multivariable plants. The parametrized expressions for the sets of restrictively stable stabilizing compensators for strongly stabilizable systems, however, by no means posssess the algebraic simplicity and transparency that conduce to usefulness in the design context. As matters now stand, the 
stability of the compensator needs to be imposed as an extemal constraint on the design procedure.

From the practical point of view, not alone uncertainty concerning the perturbation parameter but also variation in the parameters which describe the basic system model ought to be anticipated. For State-Variable described systems, the components of the State, Input and Output Matrices constitute these basic system parameters. It is to be noted in this connection that while the algebraic design based on Fractional Representation indeed safeguards against the occurrence of unstable right-half-plane exact pole-zero cancellations, this design does not rule out the possible occurrence of exact cancellations in the stable left-half-plane. Left-half-plane cancellations do not jeopardize the internal stability of the closed-loop system. But, since they render the closedloop system extremely sensitive to perturbations within the system's basic model, they can severely undermine the robustness of the design. The design procedure which aims at arbitrary pole placement in fact utilizes such left-half-plane cancellations. For this reason, Design Procedure III cannot be recommended for true practical viability, while Design Procedures I and II remain solid.

In a submodule of one of the design procedures, it was possible, in theory, to match the transfer matrices of the closed-loop slow and fast subsystems exactly to chosen transfer matrices. But in the overall design, only the closed-loop poles of the singularly perturbed plant were, approximately, matched. The reason for the limitation is that the design procedure for the singularly perturbed system is validated, specifically, by Luse's Theorem -- which addresses only the approximate location of the poles of the closed-loop system. It is true that, by Luse's Approximation Result, any point-to-point transfer relationship in the closed-loop multiple-time-scale system is approximable at low frequencies by the corresponding point-to-point transfer relationship in the slow closed-loop subsystem and at high frequencies by the corresponding point-to-point transfer relationship in the fast cvlosed-loop subsystem. But the Closed-Loop Theorem validates 
directly neither the approximate placement of the closed-loop zeros nor the approximate matching of the overall closed-loop transfer matrix to chosen transfer matrices. Placement of the poles is sufficient to inform the basic issue of stabilization, which has been treated in the dissertation. Still, the zeros -- especially right-half-plane zeros, if they exist in the plant or if they are introduced into the design by unstable stabilizing controllers -- can also shape the frequency response in fundamental ways and can affect performance characteristics on the important scores of tracking ability, sensitivity and disturbance-rejection. A further drawback of the present design procedures is thus that they deal explicitly only with the poles of the multiple time scale system.

One fundamental and abiding strength of the design procedures based on FractionalRepresentation is that they are truly multivariable design procedures. The multivariable systems, due to their algebraic complexity, pose distinct design challenges not encountered in connection with their scalar counterparts. Historically, the prototypical design techniques for the scalar systems were developed before the multivariable design problem came even rudimentarily to be understood. Many of the prevailing MIMO design procedures still reduce the multivariable problem -- either explicitly or tacitly -- to a set of scalar problems in the quest to render it tractable. But under Fractional Representation, the MIMO problem is tractable fundamentally and in its own right. Purely for the convenience of hand calculation, a simple scalar system was used in Chapter IV to illustrate the design procedures. If computer-aided design had been employed, a multivariable example might instead have been provided. The procedures remain both mathematically and computationally extremely simple in the multivariable case, requiring no further matrix operations than addition, multiplication, inversion and the determination of constant state-feedback controller and observer gains -- the most basic of operations in any available Control software. Yet the results which are promised in the MIMO case are far from trivial. It is believed that the present procedures -- even though they are imperfect -- already represent a significant design advance for the multivariable multiple-time-scale systems. 
The present design strategy for the multiple-time-scale systems, a strategy of the separate-and-composite type, was devised to possess the advantage over earlier dynamic output controller strategies for these systems of being able to handle systems with instabilities in their fast or "parasitic" dynamics alongside the usual instabilities of the slow or "normal" modes. Being a frequency-domain design strategy based on input-output system representations, it has the advantage of requiring no specific internal system model for its applicability and the disadvantage of not revealing possible "hidden" system modes. On the explicit singularly perturbed system, the far simpler time-domain separate-and-composite state-feedback design is implementable. But the system which is implicitly singularly perturbed is not in general presentable in the specific state-space description which that particular design is set up to exploit. In this light, the applicability of the present design methods to the implicit as well as to the explicit singularly perturbed system, i.e. to the broader as well as to the narrower class of linear autonomous systems which possess the multiple-time-scale property, is a further mark of merit which advances their timely commendation.

\subsection{OUTLINE OF DIRECTIONS FOR FUTURE RESEARCH}

In modern approaches, many of the problems of Automatic Control have come increasingly to be posed within abstract Ring-Theoretic settings. The Ring S(s) came to be defined by Vidyasagar out of concem for the stability of lumped linear time-invariant systems. In the study of systems of other types -- including certain classes of discrete-time, distributed, multidimensional and nonlinear system -- other Rings arise in equally "natural" ways and play the role which for the systems of the present class is played by $\mathrm{S}(\mathrm{s})$. It is desired in the general case to design a feedback system which possesses a certain stability property; therefore, a helpful first step is to model the plant, if possible, as a quotient over a Ring of operators which possess the 
property in question. The Ring structure ensures that series-parallel configurations of systems with the required property, will also have the property. In paradigm: the set of scalar stable systems of the given type forms the Ring $R$, and the set of scalar unstable (i.e. not necessarily stable) systems of the type comprises F, the Quotient Field of R. Stable multivariable systems of the type then belong to the Ring of matrices over $\mathrm{R}$, and corresponding unstable multivariable systems of the type belong to the Ring of matrices over F. Thereby, all those systems which can be expressed as scalar or matrix ratios of stable systems are encompassable within the given framework of stabilization. The notions underlying this general procedure have been formalized by Desoer, Liu, Murray and Saeks [5] and again by Desoer and Gustavson [6] into a unified and virtually axiomatic theory of feedback control system design through fractional system representation.

There thus exist specific Fractional-Representation models for discrete- and continuoustime systems of both lumped and distributed types and for certain classes of multidimensional and nonlinear system as well. The approach taken by Vidyasager in Control System Synthesis [32], which has been followed in the dissertation, reflects one choice -- the choice to work in the Rings $M(S)$ and $M(R(S))$-- within an array of possible representations under general RingTheoretic settings. The underlying Ring $\mathrm{R}$ does not in each of the specific models possess the full algebraic and topological structure of $S(s)$. In consequence, some but not all of the results for the finite-dimensional linear autonomous systems carry over to the other system classes.

The finite-dimensional linear shift-invariant discrete-time systems display themselves very handily within the setting of the present formalism. When the Z-Transform is defined as: $H(z)=\sum h_{i} z^{i}$, the set of transfer functions of the stable discrete-time systems is the Ring $R_{\infty}(z)$ of rational transfer functions in $\mathrm{z}$ having no poles inside the unit disc. The Algebra of the Euclidean Ring $R_{\infty}(z)$ derives from the degree function, $\operatorname{deg}(f)=$ number of zeros of $f$ inside the unit disc, and its Topology derives from the norm, ||$f||=\sup _{|| z|| s\rfloor}|f(z)|$. The bilinear mapping, $z=(s-$ 
$1) /(s+1)$, takes the closed right half plane into the closed unit disc. Through this mapping, $R_{\infty}$ and $\mathrm{S}$ are isometrically isomorphic as normed Algebras over the Reals. The set A, of all complexvalued functions which are analytic on the disc and which satisfy certain basic boundednessconditions, well known in mathematics as the Hardy Space over the Unit Disc (or more simply as the "Disc Algebra") is a commutative Complex Banach Algebra with Identity, a still more highly structured mathematical object. Thanks to the Functional-Analytic theory of the Hardy Spaces, the properties of $A$ are well understood. Thanks to the fact that $R_{\infty}$ is a dense subalgebra of $A$, the results which attain in A also hold -- modulo certain technical amendments -- in $\mathrm{R}_{\infty}$. Thanks, finally, to the isometric isomorphism between $R_{\infty}$ and $S$, these results must also hold in $S$. Thus, Control-Theoretic problems are, if anything, more illuminatingly treated for the discrete-time systems than for their continuous-time counterparts under the Fractional-Representation framework.

In the models for distributed systems, on the other hand, $\mathrm{R}$ fails to be a Principal Ideal Domain, and the topology defined on it fails to make $\mathrm{R}$ into a Toplogical Ring in which the set of units is an open set and inversion is a continuous operation. Consequently, the topology defined on the Ring of stable scalar distributed systems is not extendable to a topology on the set of unstable multivariable distributed systems in as natural a way as the norm topology on S(s) extends to the Graph Topology on $M(R(s))$ or as the norm topology on $R_{\infty}$ extends to a topology for the finite-dimensional discrete-time multivariable systems. These and other theoretical matters a propos the extension of results to diverse Ring-Theoretic frameworks are considered in the final Chapter of Control System Synthesis[32], and as well are treated in the paper, "Algebraic and Topological Aspects of Feedback Stabilization" by Vidyasagar, Schneider and Francis [29].

The marriage of these modem Algebraic methods to the frequency-domain study of the multiple-time-scale systems has been the basic methodological theme of the dissertation. The dissertation has studied only the continuous-time, linear, autonomous, finite-dimensional 
multiple-time-scale systems -- employing a framework which completely unifies the results for the scalar and the multivariable systems of this type. By the previous discussion, wider potentialities for unification under Fractional Representation frameworks also exist. Elsewhere in the literature, singularly perturbed discrete-time, distributed, multidimensional, time-varying and nonlinear systems have also been studied $[17,28]$. Still elsewhere in the literature, the singularly perturbed system has been examined in the contexts of optimal and stochastic control [17,28]. The Optimal Linear Quadratic Regulator problem, for example, has been solved in reduced dimensions for singularly perturbed plants. For the singularly perturbed system, the corresponding Ricatti system whose solutions implement the feedback gains which constitute the optimal linear quadratic control law is itself singularly perturbed, and the LQR problem has been approached through lower-order "slow" and "fast" Ricatti systems which approximate the full associated Ricatti system. For the singularly perturbed system subject to Gaussian disturbances, the Kalman Filter has been decomposed into filters for the respective "slow" and "fast" subsystems in an approximation of the given stochastic problem by two reduced-order stochastic problems in different time-scales. For the nonlinear singularly perturbed plant, stabilizability and stability have been analyzed in terms of separate Lyapunov functions devised for the subsystems and then composed into an appropriate Lyapunov function for the whole.

Thanks largely to the existence of the recent comprehensive review articles by Kokotovic, O'Malley and Sannuti [17] and by Saksena, O'Reilly and Kokotovic [28], such results for the singularly perturbed system as cited above are well documented in the literaure. These results reflect the singularly perturbed system, however, primarily from time-domain points of view. It is timely for the Fractional-Representation methods to attempt to provide unifying frequencydomain interpretations and extensions of the available time-domain results on any and all of these topics. $\mathrm{H}_{\mathrm{p}}$-Control Theory provides one means for performing optimization in the frequency domain which is closely meshed with the modern algebraic methods. Of the various known $\mathrm{H}_{\mathrm{p}}$ 
problem classes, the $\mathrm{H}_{\infty}$ problems, which can be conceptualized as problems pertaining to Hilbert Space Operators, are the ones presently best understood. Insofar as standard problems of filtering, sensitivity-minimization and disturbance-rejection can be cast as $\mathrm{H}_{\mathrm{p}}$-Optimization problems $[8,9,34]$, the $\mathrm{H}_{\mathrm{p}}$-Theory indeed opens up new realms for exploration pertaining to Automatic Control. The $\mathrm{H}_{\mathrm{p}}$-Theory remains to be applied distinctively to the multiple-time-scale systems, however. The dissertation, without reliance on the $\mathrm{H}_{\mathrm{p}}$-Theory, has achieved a set of solutions to some basic problems of Stabilization and Pole-Placement in connection with the multiple-time-scale system. It is hoped in future work to consider further problems a propos the multiple-time-scale system for clarification through a continuing marriage with the modem Algebraic methods, including the $\mathrm{H}_{\mathrm{p}}$-optimization methods.

The multiple-time-scale system has been discussed in the dissertation, furthermore, only in a formal way. That is to say, the dissertation has not addressed the question of how this system concretely arises in a modelling context. Indeed, each of the diverse disciplines dealing with dynamical systems has its own standard, "textbook" example of a low-order singularly perturbed system. The low-order circuit with small stray capacitances and large coupling capacitances which introduces Chapter I is one such example, taken from the context of Circuit Theory. As well, elementary Control Theory provides simple examples of systems, not themselves singularly perturbed, which behave like singularly perturbed systems under the influence of very high feedback gain or through the availability of very "cheap" control. Such small-scale examples are, naturally, easy to identify and easy to depict. But in truth, the most significant and most challenging opportunities for singular-perturbational modelling come from the domain of Large Scale Systems.

Virtually any large scale system -- be it power system, industrial plant, econometric model, ecological structure or neural network -- is aggregable into coherent subsystems which are intemally strongly coupled and only weakly linked to one another. P.V. Kokotovic and H. K. 
Khalil $[12,13]$ have shown how the weak and strong couplings of the large scale system may in general be modelled in terms of slow and fast time-scales. In their model, a "slow" core subsystem basically represents the large scale system's global, or system-wide, behavior, while a group of "fast" peripheral subsystems approximate the local behaviors of the system's relatively coherent and relatively autonomous sub-aggregations.

Through the wide applicability of this modelling method, a vital significance for the Decentralized Control of very general Large Scale Systems accrues to the Stabilization Problem for the singularly perturbed system. Kokotovic and Khalil have formally structured one situation, called "Multimodelling", in which a multiplicity of Decision Makers operating in terms of partially-overlapping decentralized system models pursue diverse and possibly competing control objectives. One goal for future work is to identify and to formally structure other modelling situations which, like Multimodelling, call for Decentralized Control, and to extend the dissertation's design procedures for applicability to them.

Independently of the dissertation, this writer has done some work in the mathematical modelling of neural networks. In this work, the networks were modelled by systems of nonlinear ordinary differential equations, which were required to exhibit the satisfactory stable behaviors of "pattern recognition", "memory", "learning", "self-organization" and ability to reach and to store "decisions". The systems were proscribed from displaying such unsatisfactory unstable behaviors as limit cycles, unending oscillations, travelling bursts and travelling waves of steadily increasing amplitude. In mathematical terms, the property sought for the models was Absolute Global Stability -- the property that each trajectory of the system approaches one and only one of a specifiable set of equillibrium points, and that no reasonable change in the system parameters destroys this global measure of stability. At one point in the model, the delays in neural signal propogation within self-excitatory and self-inhibitory feedback loops were described in terms of singular perturbations of the responses off the stimuli rather than in terms of conventional time 
delays. It is of interest to investigate whether the stabilization-techniques of the dissertation, in some appropriately modified form, can be applied to the analysis of the feedback loops in this dynamical model of a neural network.

The dissertation's argument, though unfolded through exposition, discussion and analysis rather than through formal theorem-and-proof, has dwelt basically in the realm of Theory. First and foremost it was sought to establish the theoretical possibility and the formal validity of several novel design procedures for the multiple-time-scale systems. The concluding discussion has attempted to cast the overview and evaluation of the procedures in a somewhat more practical mien. The serious study of their applicability and the question of their true practical viability remain, of course, for future exploration. Since they are already high-level algorized, the procedures are ready to be illustrated and tested in more interesting cases and on more complex examples than the dissertation itself has provided. Thereby, the computational aspects and the potentialities for software development, which have not been emphasized in the dissertation, will come into explicit focus. Alongside the several ideas for future theoretical development, the development of the computational aspects of the dissertation's design procedures stands high in priority as a subject slated to recieve attention in future work. 


\section{REFERENCES CITED}

[1] Blomberg, H. and R. Ylinen, Algebraic Theory for Multivariable Linear Systems, Academic Press, London, 1983.

[2] Chen, C.-T. Linear System Theory and Design, Holt, Rinehart and Winston, New York, 1984

[3] Coderch, M., A. Willsky, S. Sastry and D. Castanon, "Hierarchical Aggregation of Linear Systems with Multiple Time Scales", IEEE Trans. Automatic Control, Vol. AC-28, No. 11, pp 1017-1030, November 1983.

[4] Desoer, C. A. and M. J. Shensa, "Networks with Very Small and Very Large Parasitics: Natural Frequencies and Stability", IEEE Trans. Automatic Control, Vol. 58, No. 12, pp 1933-1938, December 1970.

[5] Desoer, C. A., R. Liu, J. Murray and R. Saeks, "Feedback System Design: The Fractional Representation Approach to Analysis and Synthesis", IEEE Trans. Automatic Control, Vol. AC-25, No. 3, pp 399-412, June 1980.

[6] Desoer, C. A., and C. L. Gustavson, "Algebraic Theory of Linear Multivariable Feedback Systems", IEEE Trans. Automatic Control, Vol. AC-29, No. 10, pp 909-917, October 1984.

[7] Fossard, A. J. and J. F. Magni, "A Frequential Analysis of Singularly Perturbed Systems with Static Output Control", Large Scale Systems, Vol. 1, pp 223-228, 1980.

[8] Francis, B. A. and G. Zames, "On $\mathrm{H}_{\infty}$-Optimal Sensitivity Theory for SISO Feedback Systems", IEEE Trans. Automatic Control, Vol. AC-27, No. 1, pp 9-16, January 1984.

[9] Francis, B. A., J. W. Helton and G. Zames, "Ho-Optimal Feedback Controllers for Linear Multivariable Systems", IEEE Trans. Automatic Control, Vol. AC-20, No. 10, October 1984.

[10] Kailath, T., Linear Systems, Prentice-Hall, Englewood Cliffs, New Jersey, 1980. 
[11] Kato, T., Perturbation Theory for Linear Operators, Springer-Verlag, Berlin, 1966.

[12] Khalil, H. K., and P. V. Kokotovic, "Control Strategies for Decision Makers Using Different Models of the Same System", IEEE Trans. Automatic Control, Vol. AC-23, No. 2, pp 289-297, April 1978.

[13] Khalil, H. K., and P. V. Kokotovic, "D-Stability and Multi-Parameter Singular Perturbation". SIAM J. Control and Optimization, Vol. 17, No. 1, pp 56-65, 1979.

[14] Khalil, H. K., "On the Robustness of Output Feedback Control Methods to Modelling Errors", IEEE Trans. Automatic Control, Vol. AC-26, No. 2, pp 524-526, April 1981.

[15] Khalil, H. K., "A Further Note on the Robustness of Output Feedback Control Methods to Modelling Errors", IEEE Trans. Automatic Control, Vol. AC-29, No. 9, pp 861-862, September 1984.

[16] Kokotovic, P. V., and A. H. Haddad, "Controllability and Time-Optimal Control Control of Systems with Slow and Fast Modes", IEEE Trans. Automatic Control Vol. AC-20, pp 111-113, February 1975.

[17] Kokotovic, P. V., R. E. O'Malley and P. Sannuti, "Singular Perturbations and Order Reduction in Control Theory -- An Overview", Automatica, Vol. 12. pp 123-132, 1976.

[18] Luse, D. W., "Frequency Domain Methods for Systems with Slow and Fast Dynamics", Ph.D. Dissertation, Michigan State University, 1983.

[19] Luse, D. W. and H. K. Khalil, "A Frequency Domain Approach for Systems with Slow and Fast Modes", Proc. American Control Conference, pp 443-444, 1983.

[20] Luse, D. W., and H. K. Khalil, "Frequency Domain Results for Systems with Slow and Fast Dynamics", IEEE Trans. Automatic Control, Vol. AC-30, No. 12, pp 1171-1179, December 1985.

[21] Luse, D. W., "Frequency Domain Results for Systems with Multiple Time Scales", IEEE Trans. Automatic Control, Vol. AC-31, No. 10, pp 918-924, October 1986.

[22] Nett, C. N., C. A. Jacobson and M. J. Balas, "A Connection Between State-Space and Doubly Coprime Fractional Representations', IEEE Trans. Automatic Control, Vol. AC29, No. 9, pp 831-832, September 1984.

[23] O'Reilly, J., "Robustness of Linear Feedback Control Systems to Unmodelled HighFrequency Dynamics", Int. J. Control, Vol. 44, No. 4, pp 1077-1088, 1986.

[24] Pernebo, L., "An Algebraic Theory for the Design of Controllers for Linear Multivariable Systems -- Part I: Structure Matrices and Feedforward Design", IEEE Trans. Automatic Control, Vol. AC-26, No. 1, pp 171-182, February 1981. 
[25] Pernebo, L., "An Algebraic Theory for the Design of Controllers for Linear Multivariable Systems -- Part II: Feedback Realizations and Feedback Design", IEEE Trans. Automatic Control, Vol. AC-26, No. 1, pp 183-194, February 1981.

[26] Porter, B. and A. T. Shenton, " Singular Perturbation Analysis of the Transfer Function Matrices of a Class of Multivariable Linear Systems", Int. J. Control, Vol. 21, No. 4, pp 655-660, 1975.

[27] Rosenbrock, H. H., State-Space and Multivariable Theory, John Wiley \& Sons, New York, 1970.

[28] Saksena, V. R., J. O'Reilly and P. V. Kokotovic, "Singular Perturbations and Time-Scale Methods in Control Theory: Survey 1976-1983", Automatica, Vol. 20, No. 3, pp 273293, 1984.

[29] Vidyasagar, M., H. Schneider and B. A. Francis, "Algebraic and Topological Aspects of Feedback Stabilization", IEEE Trans. Automatic Control, Vol. AC-27, No. 4, pp 888894, August, 1982.

[30] Vidyasagar, M. and N. Viswanadham, "Algebraic Design Techniques for Reliable Stabilization", IEEE Trans. Automatic Control, Vol. AC-27, No. 5, pp 1085-1095, October 1982.

[31] Vidyasagar, M., "The Graph Metric for Unstable Plants and Robustness Estimates for Feedback Stability", IEEE Trans. Automatic Control, Vol. AC-29, No. 5, pp 403-417, May 1984.

[32] Vidyasagar, M., Control System Synthesis: A Factorization Approach, MIT Press, Cambridge, Massachusetts, 1985.

[33] Vidyasagar, ıvi., "Robust Stabilization of Singularly Perturbed Systems", Systs. Contr. Letts., Vol. 5, No. 6, pp 413-418, May 1985.

[34] Vidyasagar, M., "Optimal Rejection of Persistent Bounded Disturbances", IEEE Trans. Automatic Control, Vol. AC-31, No. 6, pp 527-534, June 1986. 